Supporting Information for

\title{
Exploring Overall Photoelectric Applications by Organic Materials Containing Symmetric Donor Isomers
}

\author{
Zhaoyang Yao, ${ }^{\dagger}, \circ$ Xunfan Liao, $, \#, \circ$ Yaxiao Guo, ${ }^{\dagger}, \circ$ Heng Zhao, ${ }^{\S}$ Yu Guo, ${ }^{\dagger}$ Fuguo Zhang, ${ }^{\dagger}$ Lin Zhang, ${ }^{\S, \nabla}$ Zonglong \\ Zhu, ${ }^{\perp}$ Lars Kloo, ${ }^{\dagger}$ Wei Ma, ${ }^{*}, \S$ Yiwang Chen, $, *, * \#$ and Licheng Sun $*, \dagger, \|$ \\ $\dagger$ Department of Chemistry, Organic/Applied Physical Chemistry, KTH Royal Institute of Technology, \\ Stockholm, 10044, Sweden \\ $¥$ State Key Laboratory for Modification of Chemical Fibers and Polymer Materials \& College of \\ Materials Science and Engineering, Donghua University, Shanghai 201620, China \\ $\S$ State Key Laboratory for Mechanical Behavior of Materials, Xi'an Jiaotong University, Xi' an, 710049, \\ China \\ ॥ State Key Laboratory of Fine Chemicals, Institute of Artificial Photosynthesis, DUT-KTH Joint \\ Education and Research Centre on Molecular Devices, Dalian University of Technology (DUT), Dalian, \\ 116024, China \\ $\perp$ Department of Biology and Chemistry, City University of Hong Kong, Kowloon, 999077, Hong Kong \\ \# Institute of Advanced Scientific Research, Jiangxi Normal University, 99 Ziyang Avenue, Nanchang, \\ 330022, China \\ $\nabla$ Hunan Key Laboratory for Super Microstructure and Ultrafast Process, School of Physics and \\ Electronics, Central South University, Changsha, 410083, China
}

\section{Table of Contents}

1. Experimental Section

1.1. Materials and Synthesis pp. S2-S27

1.2. Instrumentation pp. S28

1.3. Theoretical Calculations. pp. S28

1.4. Device Fabrication .pp. S28-S29

1.5. Characterization of solar cells ..pp. S29

1.6. SCLC measurements .pp. S29-S30

1.7. Grazing Incidence Wide-Angle X-ray Scattering (GIWAXS) Characterization. pp. $\mathrm{S} 30$

2. Additional Figures and Tables. pp. S31-S49

3. Spectral Charts of NMR and IR .pp. S50-S97

4. References pp. S98-S99 


\section{EXPERIMENTAL SECTION}

1.1 Materials. Tetrabutylammonium hexafluorophosphate $\left(\mathrm{Bu}_{4} \mathrm{NPF}_{6}\right)$, ferrocene (FC), 2isopropoxy-4,4,5,5-tetramethyl-1,3,2-dioxaborolane, tetrakis(triphenylphosphine)palladium $\left(\mathrm{Pd}\left(\mathrm{PPh}_{3}\right)_{4}\right), \quad$ palladium(II) acetate $\left(\mathrm{Pd}(\mathrm{OAc})_{2}\right)$, tricyclohexylphosphinetetrafluroborate $\left(\mathrm{PCy}_{3} \cdot \mathrm{HBF}_{4}\right)$, trimethylacetic acid $(\mathrm{PivOH})$, boron trifluoride-diethyl etherate, methyl thiophene3-carboxylate, methyl 2-bromothiophene-3-carboxylate, 3-(bromomethyl)heptane, 1bromohexane, $n$-butyllithium (n-BuLi), 1-bromo-4-hexylbenzene, 2-(5,6-difluoro-3-oxo-2,3dihydro- $1 H$-inden-1-ylidene)malononitrile, 1,3-diethyl-2-thioxodihydropyrimidine-4,6(1H,5H)dione, $\beta$-alanine, 18-crown-6 and potassium carbonate $\left(\mathrm{K}_{2} \mathrm{CO}_{3}\right)$ were purchased from SigmaAldrich. Phosphorus oxychloride ( $\left.\mathrm{POCl}_{3}\right), N, N$-dimethylformide (DMF), dichloromethane (DCM), acetonitrile (AN), tetrahydrofuran (THF), 1,2-dichloroethane (DCE), toluene and ethanol were dried and distilled before use. 2-(5,6-Difluoro-3-oxo-2,3-dihydro-1H-inden-1ylidene)malononitrile ${ }^{[\mathrm{S} 1]}$ and butyl 4-((7-bromobenzo[c][1,2,5]thiadiazol-4-yl)ethynyl)benzoate ${ }^{[\mathrm{S} 2]}$ were synthesized according to the respective literature procedures. Other chemicals were purchased and used without further purification. The synthetic routes to T1 and T2 were illustrated in Scheme 1. The synthetic routes to T1-C6 were illustrated in Scheme S1. The synthetic routes to TB1, TB2, TF1 and TF2 were illustrated in Scheme 2. The synthetic routes to TB1-C6 and TF1-C6 were illustrated in Scheme S2. The synthetic routes to DT1 and DT2 were illustrated in Scheme 3. The preparation details were described as follows.

5-Bromo-1-(2-ethylhexyl)indolin-2-one (2)

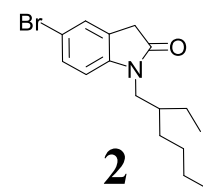

We prepared compound $\mathbf{2}$ according to the literature with some modifications. ${ }^{[\mathrm{S} 3]} \mathbf{1}$ (5.00 g, 23.58 mmol), 3-(bromomethyl)heptane (5.01 g, $25.94 \mathrm{mmol}), \mathrm{K}_{2} \mathrm{CO}_{3}(3.58 \mathrm{~g}, 25.94 \mathrm{mmol})$ and 18crown-6 (6.85 g, $25.94 \mathrm{mmol})$ were dissolved in acetonitrile $(100 \mathrm{~mL})$ in a three-neck roundbottom flask. The resulting mixture was stirred for $48 \mathrm{~h}$ at $80{ }^{\circ} \mathrm{C}$ and then cooled to room temperature. Dichloromethane $(80 \mathrm{~mL})$ was added and the organic layer was washed with water $(80 \mathrm{~mL} \times 3)$ and dried over anhydrous sodium sulfate. After removing solvent under reduced 
pressure, the residue was purified by column chromatography (ethyl acetate/petroleum ether 40$\left.60{ }^{\circ} \mathrm{C}, 1 / 10, v / v\right)$ on silica gel to afford a colorless oil as the desired product 2 (5.58 g, $73 \%$ yield). 3,8,13-Tribromo-5,10,15-tris(2-ethylhexyl)-10,15-dihydro-5H-diindolo[3,2-a:3',2'-c]carbazole (3)

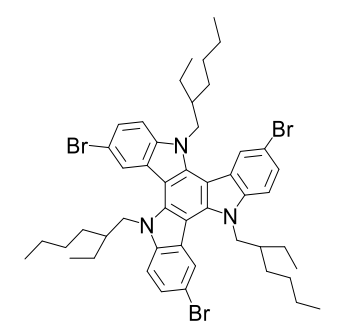

3

$2(5.00 \mathrm{~g}, 15.43 \mathrm{mmol})$ was dissloved in $\mathrm{POCl}_{3}(50 \mathrm{~mL})$ and the resulting mixture was stirred for $20 \mathrm{~h}$ at $100{ }^{\circ} \mathrm{C}$ and then cooled to room temperature. The excessive $\mathrm{POCl}_{3}$ was distilled under vacuum and the residue was meutralized by $\mathrm{KOH}$ aqueous solution. Dichloromethane ( $80 \mathrm{~mL})$ was added and the organic layer was washed with water $(80 \mathrm{~mL} \times 3)$ and dried over anhydrous sodium sulfate. After removing solvent under reduced pressure, the residue was purified by column chromatography $\left(\mathrm{DCM} /\right.$ petroleum ether $\left.40-60{ }^{\circ} \mathrm{C}, 1 / 8, v / v\right)$ on silica gel to afford a white solid as the desired product $3\left(1.65 \mathrm{~g}, 36 \%\right.$ yield). ${ }^{1} \mathrm{H}$ NMR (400 MHz, $\left.\mathrm{CDCl}_{3}\right) \delta: 7.96(\mathrm{~d}, J=8.5 \mathrm{~Hz}, 3 \mathrm{H})$, $7.63(\mathrm{~s}, 3 \mathrm{H}), 7.44$ (d, J=8.5 Hz, 3H), 4.61 (br, 6H), 1.89 (br, 3H), 0.98-0.90 (m, 12H), 0.86-0.78 $(\mathrm{m}, 9 \mathrm{H}), 0.73-0.63(\mathrm{~m}, 12 \mathrm{H}), 0.47-0.37(\mathrm{~m}, 9 \mathrm{H})$. The characterization of ${ }^{13} \mathrm{C} \mathrm{NMR}$ and MS are the same with literature. ${ }^{[\mathrm{S} 4]}$

5,10,15-Tris(2-ethylhexyl)-3,8,13-tris(4,4,5,5-tetramethyl-1,3,2-dioxaborolan-2-yl)-10,15dihydro-5H-diindolo[3,2-a:3',2'-c]carbazole (4)

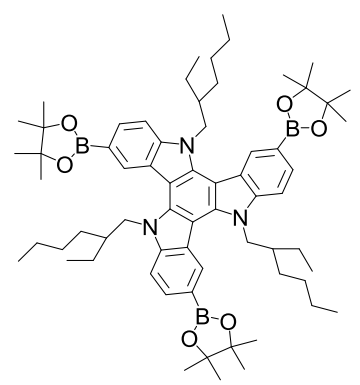

4 
$3(1.00 \mathrm{~g}, 1.09 \mathrm{mmol})$ was dissolved in THF $(15 \mathrm{~mL})$ in a three-neck flame-dried round-bottom flask. The solution was cooled to $-78{ }^{\circ} \mathrm{C}$ using a dry ice/acetone cold bath before the addition of $n$-BuLi (1.74 mL, 2.5 M in hexanes, $4.36 \mathrm{mmol})$. The reaction mixture was stirred for $1 \mathrm{~h}$ at -78 ${ }^{\circ} \mathrm{C}$. After the addition of 2-isopropoxy-4,4,5,5-tetramethyl-1,3,2-dioxaborolane $(811 \mathrm{mg}, 3.49$ $\mathrm{mmol}$ ) in one portion via syringe, the mixture was slowly warmed up and stirred for $2 \mathrm{~h}$ at room temperature. Water $(10 \mathrm{~mL})$ was slowly added to terminate the reaction and the mixture was extracted three times with chloroform $(50 \mathrm{~mL} \times 3)$ before the organic phase was washed with water and dried over anhydrous sodium sulfate. After solvent removal under reduced pressure, the crude product 4 was used in the subsequent reaction directly. Yield $(\sim 82 \%)$.

Trimethyl 2,2',2"-(5,10,15-tris(2-ethylhexyl)-10,15-dihydro-5H-diindolo[3,2-a:3',2'-c]carbazole3,8,13-triyl)tris(thiophene-3-carboxylate) (5)

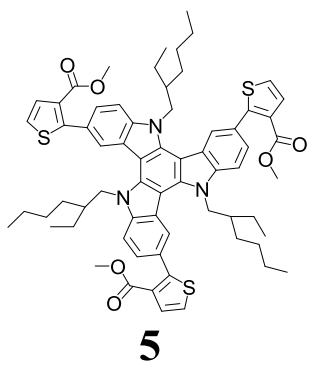

Method I: In a two-neck round-bottom flask, compound 4 (500 mg, $0.47 \mathrm{mmol}$ ), methyl 2bromothiophene-3-carboxylate $(415 \mathrm{mg}, 1.88 \mathrm{mmol}), \mathrm{Pd}\left(\mathrm{PPh}_{3}\right)_{4}(58 \mathrm{mg}, 0.05 \mathrm{mmol})$ and $\mathrm{K}_{2} \mathrm{CO}_{3}$ (648 mg, $4.70 \mathrm{mmol})$ were dissolved in a mixture of solvent (toluene/ $\mathrm{H}_{2} \mathrm{O} /$ ethanol, 1/1/0.2, v/v/v) under nitrogen. The resulting mixture was stirred at reflux overnight before cooling down to room temperature. Chloroform $(50 \mathrm{~mL})$ was added and the organic layer was washed with brine $(50 \mathrm{~mL}$ $\times 3)$ and dried over anhydrous sodium sulfate. Then remove the desiccant by filtering and the filtrate was concentrated under reduced pressure. The residue was purified by column chromatography $\left(\mathrm{DCM} /\right.$ petroleum ether $\left.40-60{ }^{\circ} \mathrm{C}, 4 / 1, v / v\right)$ on silica gel to yield a light yellow solid as the desired product 5 (631 $\mathrm{mg}, 80 \%$ yield).

Method II: In a dried two-neck round-bottom flask were dissolved compound 3 (1.00 g, 1.09 $\mathrm{mmol})$, methyl thiophene-3-carboxylate $(619 \mathrm{mg}, 4.36 \mathrm{mmol})$ and $\mathrm{K}_{2} \mathrm{CO}_{3}(1.64 \mathrm{~g}, 11.87 \mathrm{mmol})$ in toluene $(30 \mathrm{~mL})$. Then $\mathrm{Pd}(\mathrm{OAc})_{2}(136 \mathrm{mg}, 0.48 \mathrm{mmol}), \mathrm{PCy}_{3} \cdot \mathrm{HBF}_{4}(342 \mathrm{mg}, 0.94 \mathrm{mmol})$, and $\mathrm{PivOH}(251 \mathrm{mg}, 2.38 \mathrm{mmol})$ were added to the reaction mixture in a nitrogen-filled glovebox, 
which was refluxed for $48 \mathrm{~h}$. The mixture was extracted three times with chloroform $(30 \mathrm{~mL} \times 3)$ before the organic phase was washed with water $(80 \mathrm{~mL} \times 3)$ and dried over anhydrous sodium sulfate. After solvent removal under reduced pressure, the crude product was purified by column chromatography (DCM/petroleum ether $\left.40-60{ }^{\circ} \mathrm{C}, 4 / 1, v / v\right)$ on silica gel to yield a yellow solid as the desired product 5 (852 mg, 71\% yield). ${ }^{1} \mathrm{H}$ NMR (400 MHz, $\left.\mathrm{CDCl}_{3}\right) \delta: 8.23$ (br, 3H), 7.77 (br, 3H), 7.59 (d, J = 5.4 Hz, 3H), 7.51 (br, 3H), 7.30 (d, J = 5.4 Hz, 3H), 4.87 (br, 6H), 3.77 (s, 9H), 2.12 (br, 3H), 1.04-0.86 (m, 24H), 0.68-0.61 (m, 9H), 0.54-0.50 (m, 9H). ${ }^{13} \mathrm{C}$ NMR (125 MHz, $\left.\mathrm{CDCl}_{3}\right) \delta: 164.07,152.75,140.27,139.54,130.41,127.04,126.83,123.70,123.39,121.64,121.17$, 112.99, 102.99, 51.71, 50.38, 37.97, 37.88, 29.73, 28.34, 28.23, 28.10, 23.02, 22.81, 13.87, 10.27, 10.20, 9.59. HR-MS (MALDI-TOF) $\mathrm{m} / z$ calcd. for $\left(\mathrm{C}_{66} \mathrm{H}_{75} \mathrm{~N}_{3} \mathrm{O}_{6} \mathrm{~S}_{3}\right):$ 1101.48180. Found: 1101.48189. Anal. Calcd. for $\mathrm{C}_{66} \mathrm{H}_{75} \mathrm{~N}_{3} \mathrm{O}_{6} \mathrm{~S}_{3}: \mathrm{C}, 71.90 \%$; H, 6.86\%; N, 3.81\%. Found: C, 71.96\%; $\mathrm{H}, 6.85 \% ; \mathrm{N}, 3.82 \%$.

6,13,20-Tris(2-ethylhexyl)-4,4,11,11,18,18-hexakis(4-hexylphenyl)-4,6,11,13,18,20hexahydrothieno[3",2":4',5']cyclopenta[1',2':5,6]indolo[3,2 a]thieno[3", 2": 4',5']cyclopenta[1',2':5,6]indolo[3,2-c]thieno[3',2':4,5]cyclopenta[1,2h]carbazole $(\boldsymbol{T} 1)$

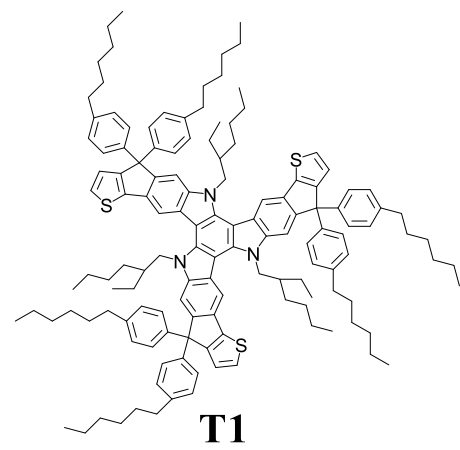

In a flame-dried two-neck flask were added $5(400 \mathrm{mg}, 0.36 \mathrm{mmol})$ and $30 \mathrm{~mL}$ anhydrous THF and the mixture was cooled to $0^{\circ} \mathrm{C}$ using ice-salt baths. Then (4-hexylphenyl)lithium ( $3 \mathrm{~mL}, 1 \mathrm{M}$ in THF, $3.00 \mathrm{mmol}$ ) was injected one portion via syringe before the resulting solution was heated to room temperature. The mixture was further stirred at room temperature for $1 \mathrm{~h}$ and then $1 \mathrm{M} \mathrm{HCl}$ aqueous solution $(20 \mathrm{~mL})$ was added to terminate the reaction. Chloroform $(60 \mathrm{~mL})$ was added and the organic layer was washed with brine $(40 \mathrm{~mL} \times 3)$ and dried over anhydrous sodium sulfate. After desiccant removal by filtering, the filtrate was concentrated under reduced pressure. The 
crude product was used in the subsequent reaction directly. In a flame-dried two-neck flask, the above tertiary alcohol was added into $30 \mathrm{~mL}$ anhydrous DCM. The resulting mixture was cooled to $0{ }^{\circ} \mathrm{C}$ before $0.2 \mathrm{~mL}$ boron trifluoride-diethyl etherate was added. After stirred at $0{ }^{\circ} \mathrm{C}$ for $10 \mathrm{~min}$, the solution was quenched by $1 \mathrm{M} \mathrm{K}_{2} \mathrm{CO}_{3}$ aqueous solution $(50 \mathrm{~mL})$. Then the mixture was extracted three times with chloroform $(30 \mathrm{~mL} \times 3)$ before the organic phase was washed with water and dried over anhydrous sodium sulfate. After solvent removal under reduced pressure, the crude product was purified by column chromatography (DCM/petroleum ether $\left.40-60{ }^{\circ} \mathrm{C}, 1 / 10, v / v\right)$ on silica gel to yield a yellow solid as the desired product T1 (617 mg, 89\% yield). ${ }^{1} \mathrm{H}$ NMR (500 $\left.\mathrm{MHz}, \mathrm{CDCl}_{3}\right) \delta: 8.39(\mathrm{~s}, 3 \mathrm{H}), 7.80(\mathrm{~s}, 3 \mathrm{H}), 7.48-7.39(\mathrm{~m}, 15 \mathrm{H}), 7.31-7.21(\mathrm{~m}, 15 \mathrm{H}), 4.93$ (br, 6H), $2.74(\mathrm{~d}, J=7.6 \mathrm{~Hz}, 12 \mathrm{H}), 2.17$ (br, 3H), 1.78-1.75 (m, 12H), 1.47 (br, 36H), 1.18-1.04 (m, 30H), 0.92-0.77 (m, 21H), 0.66-0.50 (m, 9H). $\left.{ }^{13} \mathrm{C} \mathrm{NMR} \mathrm{(125} \mathrm{MHz,} \mathrm{CDCl}_{3}\right) \delta: 156.25,146.29,143.45$, 141.94, 141.20, 140.96, 139.06, 132.65, 128.42, 128.09, 127.99, 127.08, 123.52, 121.95, 120.40, 104.53, 102.40, 62.71, 50.61, 50.53, 37.84, 37.70, 36.78, 31.93, 31.91, 31.58, 31.52, 29.37, 28.36, 28.21, 28.16, 27.99. HR-MS (MALDI-TOF) $\mathrm{m} / \mathrm{z}$ calcd. for $\left(\mathrm{C}_{135} \mathrm{H}_{165} \mathrm{~N}_{3} \mathrm{~S}_{3}\right)$ : 1925.21992 . Found: 1925.21946. Anal. Calcd. for $\mathrm{C}_{135} \mathrm{H}_{165} \mathrm{~N}_{3} \mathrm{~S}_{3}: \mathrm{C}, 84.19 \% ; \mathrm{H}, 8.64 \%$; N, 2.18\%. Found: C, 84.12\%; $\mathrm{H}, 8.68 \%$; N $2.17 \%$.

6-Bromo-1-(2-ethylhexyl)indolin-2-one (7)

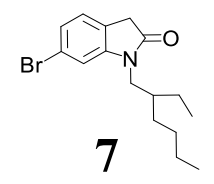

We prepared compound 7 according to the literature with some modifications. ${ }^{[\mathrm{S} 3]} 6$ (4.00 g, 18.86 mmol), 3-(bromomethyl)heptane (4.01 g, $20.75 \mathrm{mmol}), \mathrm{K}_{2} \mathrm{CO}_{3}(2.86 \mathrm{~g}, 20.75 \mathrm{mmol}$ ) and 18crown-6 (5.48 g, $20.75 \mathrm{mmol})$ were dissolved in acetonitrile $(90 \mathrm{~mL})$ in a three-neck round-bottom flask. The resulting mixture was stirred for $48 \mathrm{~h}$ at $80{ }^{\circ} \mathrm{C}$ and then cooled to room temperature. Dichloromethane $(80 \mathrm{~mL})$ was added and the organic layer was washed with water $(80 \mathrm{~mL} \times 3)$ and dried over anhydrous sodium sulfate. After removing solvent under reduced pressure, the residue was purified by column chromatography (ethyl acetate/petroleum ether $40-60{ }^{\circ} \mathrm{C}, 1 / 10$, $v / v$ ) on silica gel to afford a colorless oil as the desired product 7 (4.34 g, 71\% yield). The characterization of NMR and MS are the same with literature. ${ }^{[\mathrm{S} 5]}$ 


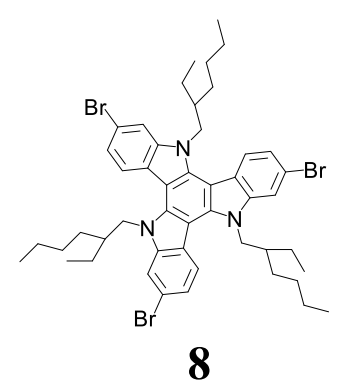

$7(4.00 \mathrm{~g}, 12.34 \mathrm{mmol})$ was dissloved in $\mathrm{POCl}_{3}(40 \mathrm{~mL})$ and the resulting mixture was stirred for $20 \mathrm{~h}$ at $100{ }^{\circ} \mathrm{C}$ and then cooled to room temperature. The excessive $\mathrm{POCl}_{3}$ was distilled under vacuum and the residue was meutralized by $\mathrm{KOH}$ aqueous solution. Dichloromethane $(50 \mathrm{~mL})$ was added and the organic layer was washed with water $(50 \mathrm{~mL} \times 3)$ and dried over anhydrous sodium sulfate. After removing solvent under reduced pressure, the residue was purified by column chromatography $\left(\mathrm{DCM} /\right.$ petroleum ether $\left.40-60{ }^{\circ} \mathrm{C}, 1 / 8, v / v\right)$ on silica gel to afford a white solid as the desired product $8(1.32 \mathrm{~g}, 36 \%$ yield $) .{ }^{1} \mathrm{H}$ NMR $\left(500 \mathrm{MHz}, \mathrm{CDCl}_{3}\right) \delta: 7.86(\mathrm{~d}, J=8.5 \mathrm{~Hz}, 3 \mathrm{H})$, 7.54 (s, 3H), 7.45 (d, $J=8.5 \mathrm{~Hz}, 3 \mathrm{H}), 4.49-4.37$ (m, 6H), 1.82-1.81 (m, 3H), 0.98-0.95 (m, 12H), $0.88-0.72(\mathrm{~m}, 9 \mathrm{H}), 0.68-0.62(\mathrm{~m}, 12 \mathrm{H}), 0.43-0.32(\mathrm{~m}, 9 \mathrm{H})$. The characterization of ${ }^{13} \mathrm{C}$ NMR and MS are the same with literature. ${ }^{[55]}$

5,10,15-Tris(2-ethylhexyl)-2,7,12-tris(4,4,5,5-tetramethyl-1,3,2-dioxaborolan-2-yl)-10,15dihydro-5H-diindolo[3,2-a:3',2'-c]carbazole (9)

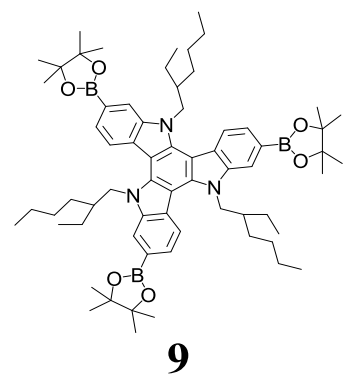

8 (900 mg, $0.98 \mathrm{mmol}$ ) was dissolved in THF (12 mL) in a three-neck flame-dried round-bottom flask. The solution was cooled to $-78{ }^{\circ} \mathrm{C}$ using a dry ice/acetone cold bath before the addition of $n$-BuLi $(1.57 \mathrm{~mL}, 2.5 \mathrm{M}$ in hexanes, $3.92 \mathrm{mmol})$. The reaction mixture was stirred for $1 \mathrm{~h}$ at -78 ${ }^{\circ} \mathrm{C}$. After the addition of 2-isopropoxy-4,4,5,5-tetramethyl-1,3,2-dioxaborolane (730 mg, 3.14 
$\mathrm{mmol}$ ) in one portion via syringe, the mixture was slowly warmed up and stirred for $2 \mathrm{~h}$ at room temperature. Water $(30 \mathrm{~mL})$ was slowly added to terminate the reaction and the mixture was extracted three times with chloroform $(50 \mathrm{~mL} \times 3)$ before the organic phase was washed with water and dried over anhydrous sodium sulfate. After solvent removal under reduced pressure, the crude product 9 was used in the subsequent reaction directly. Yield ( 69\%).

Trimethyl 2,2',2"-(5,10,15-tris(2-ethylhexyl)-10,15-dihydro-5H-diindolo[3,2-a:3',2'-c]carbazole2,7,12-triyl)tris(thiophene-3-carboxylate) (10)

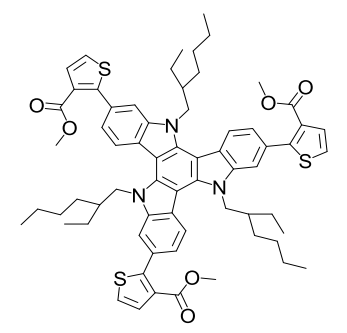

10

Method I: In a two-neck round-bottom flask, compound 9 (300 mg, $0.28 \mathrm{mmol})$, methyl 2bromothiophene-3-carboxylate $(249 \mathrm{mg}, 1.13 \mathrm{mmol}), \mathrm{Pd}\left(\mathrm{PPh}_{3}\right)_{4}(46 \mathrm{mg}, 0.04 \mathrm{mmol})$ and $\mathrm{K}_{2} \mathrm{CO}_{3}$ (389 mg, $2.82 \mathrm{mmol}$ ) were dissolved in a mixture of solvent $\left(20 \mathrm{~mL}\right.$, toluene/ $\mathrm{H}_{2} \mathrm{O} /$ ethanol, 1/1/0.2, $v / v / v)$ under nitrogen. The resulting mixture was stirred at reflux overnight before cooling down to room temperature. Chloroform $(50 \mathrm{~mL})$ was added and the organic layer was washed with brine $(50 \mathrm{~mL} \times 3)$ and dried over anhydrous sodium sulfate. Then remove the desiccant by filtering and the filtrate was concentrated under reduced pressure. The residue was purified by column chromatography $\left(\mathrm{DCM} /\right.$ petroleum ether $\left.40-60^{\circ} \mathrm{C}, 5 / 1, v / v\right)$ on silica gel to yield a light yellow solid as the desired product $\mathbf{1 0}(244 \mathrm{mg}, 79 \%$ yield $)$.

Method II: In a dried two-neck round-bottom flask were dissolved compound 8 (900 mg, 0.98 $\mathrm{mmol})$, methyl thiophene-3-carboxylate $(557 \mathrm{mg}, 3.92 \mathrm{mmol})$ and $\mathrm{K}_{2} \mathrm{CO}_{3}(1.48 \mathrm{~g}, 10.68 \mathrm{mmol})$ in toluene (30 mL). Then $\mathrm{Pd}(\mathrm{OAc})_{2}(136 \mathrm{mg}, 0.48 \mathrm{mmol}), \mathrm{PCy}_{3} \cdot \mathrm{HBF}_{4}(342 \mathrm{mg}, 0.94 \mathrm{mmol})$, and PivOH (251 mg, $2.38 \mathrm{mmol})$ were added to the reaction mixture in a nitrogen-filled glovebox, which was refluxed for $48 \mathrm{~h}$. Then the resulting solution cooled down to room temperature and chloroform $(50 \mathrm{~mL})$ was added. The organic layer was washed with brine $(50 \mathrm{~mL} \times 3)$ and dried over anhydrous sodium sulfate. After solvent removal under reduced pressure, the crude product was purified by column chromatography (DCM/petroleum ether $\left.40-60{ }^{\circ} \mathrm{C}, 5 / 1, v / v\right)$ on silica gel 
to yield a yellow solid as the desired product 10 (568 mg, 68\% yield). ${ }^{1} \mathrm{H}$ NMR $\left(500 \mathrm{MHz}, \mathrm{CDCl}_{3}\right)$ $\delta: 8.10(\mathrm{br}, 3 \mathrm{H}), 7.66$ (s, 3H), 7.62-7.60 (m, 6H), 7.29 (d, J = 5.4 Hz, 3H), 4.61 (br, 6H), 3.82 (s, 9H), 2.01 (br, 3H), 1.00-0.86 (m, 16H), 0.76 (br, 6H), 0.65-0.50 (m, 11H), 0.51-0.37 (m, 9H). ${ }^{13} \mathrm{C}$ NMR (125 MHz, $\left.\mathrm{CDCl}_{3}\right) \delta: 164.08,152.65,140.33,139.60,135.16,130.37,127.96,127.13$, $123.78,121.71,121.32,112.97,103.01,51.56,50.45,37.98,29.83,28.23,28.11,22.84,22.81$, 22.77, 13.89, 13.87, 13.86, 13.83, 10.22, 10.09, 9.99, 9.87. HR-MS (MALDI-TOF) $\mathrm{m} / \mathrm{z}$ calcd. for $\left(\mathrm{C}_{66} \mathrm{H}_{75} \mathrm{~N}_{3} \mathrm{O}_{6} \mathrm{~S}_{3}\right): 1101.48180$. Found: 1101.48167. Anal. Calcd. for $\mathrm{C}_{66} \mathrm{H}_{75} \mathrm{~N}_{3} \mathrm{O}_{6} \mathrm{~S}_{3}: \mathrm{C}, 71.90 \% ; \mathrm{H}$, $6.86 \%$; N, 3.81\%. Found: C, 71.85\%; H, 6.90\%; N, 3.79\%.

6,13,20-Tris(2-ethylhexyl)-4,4,11,11,18,18-hexakis(4-hexylphenyl)-4,6,11,13,18,20hexahydrothieno[2", 3": 3',4']cyclopenta[1',2':5,6]indolo[3,2 -

a]thieno[2", $\left.3^{\prime \prime}: 3^{\prime}, 4^{\prime}\right]$ cyclopenta[ $\left.1^{\prime}, 2^{\prime}: 5,6\right]$ indolo[3,2-c]thieno[2',3':3,4]cyclopenta[1,2h]carbazole (T2)

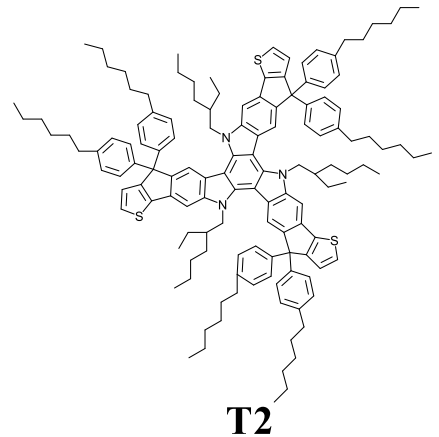

In a flame-dried two-neck flask were added $10(480 \mathrm{mg}, 0.43 \mathrm{mmol})$ and $30 \mathrm{~mL}$ anhydrous THF and the mixture was cooled to $0{ }^{\circ} \mathrm{C}$ using ice-salt baths. Then (4-hexylphenyl)lithium $(3.6 \mathrm{~mL}, 1$ $\mathrm{M}$ in THF, $3.60 \mathrm{mmol}$ ) was injected one portion via syringe before the resulting solution was heated to room temperature. The mixture was further stirred at room temperature for $1 \mathrm{~h}$ and then $1 \mathrm{M} \mathrm{HCl}$ aqueous solution $(20 \mathrm{~mL})$ was added to terminate the reaction. Chloroform $(60 \mathrm{~mL})$ was added and the organic layer was washed with brine $(50 \mathrm{~mL} \times 3)$ and dried over anhydrous sodium sulfate. After desiccant removal by filtering, the filtrate was concentrated under reduced pressure. The crude product was used in the subsequent reaction directly. In a flame-dried two-neck flask, the above tertiary alcohol was added into $30 \mathrm{~mL}$ anhydrous DCM. The resulting mixture was cooled to $0{ }^{\circ} \mathrm{C}$ before $0.2 \mathrm{~mL}$ boron trifluoride-diethyl etherate was added. After stirred at $0{ }^{\circ} \mathrm{C}$ for $10 \mathrm{~min}$, the solution was quenched by $1 \mathrm{M} \mathrm{K}_{2} \mathrm{CO}_{3}$ aqueous solution $(50 \mathrm{~mL})$. Then the mixture 
was extracted three times with chloroform $(30 \mathrm{~mL} \times 3)$ before the organic phase was washed with water and dried over anhydrous sodium sulfate. After solvent removal under reduced pressure, the crude product was purified by column chromatography (DCM/petroleum ether $\left.40-60{ }^{\circ} \mathrm{C}, 1 / 10, v / v\right)$ on silica gel to yield a yellow solid as the desired product T2 (728 mg, 88\% yield). ${ }^{1} \mathrm{H}$ NMR (500 $\left.\mathrm{MHz}_{\mathrm{CDCl}}\right) \delta: 8.12(\mathrm{~s}, 3 \mathrm{H}), 7.54(\mathrm{~s}, 3 \mathrm{H}), 7.27-7.14(\mathrm{~m}, 14 \mathrm{H}), 7.03-6.96(\mathrm{~m}, 14 \mathrm{H}), 4.73-4.63$ (m, 6H), 2.49 (d, J=8.0 Hz, 12H), $1.91(\mathrm{br}, 3 \mathrm{H}), 1.53-1.51(\mathrm{~m}, 12 \mathrm{H}), 1.28-1.22(\mathrm{~m}, 36 \mathrm{H}), 0.92-$ $0.79(\mathrm{~m}, 30 \mathrm{H}), 0.60-0.51(\mathrm{~m}, 21 \mathrm{H}), 0.34-0.24(\mathrm{~m}, 9 \mathrm{H}) .{ }^{13} \mathrm{C} \mathrm{NMR}\left(125 \mathrm{MHz}, \mathrm{CDCl}_{3}\right) \delta: 156.22$, 146.24, 143.40, 141.91, 141.17, 141.03, 140.94, 139.02, 139.97, 132.60, 128.37, 128.04, 127.95, 127.03, 123.48, 121.90, 120.37, 120.35, 104.56, 104.48, 102.35, 62.67, 50.58, 50.49, 37.80, 37.66, $35.73,31.88,31.53,31.47,29.35,29.33,28.11,27.95,22.74,14.22,14.07,14.01,10.19$, 10.00, 9.80, 9.65. HR-MS (MALDI-TOF) $m / z$ calcd. for $\left(\mathrm{C}_{135} \mathrm{H}_{165} \mathrm{~N}_{3} \mathrm{~S}_{3}\right)$ : 1925.21992. Found: 1925.21968. Anal. Calcd. for $\mathrm{C}_{135} \mathrm{H}_{165} \mathrm{~N}_{3} \mathrm{~S}_{3}: \mathrm{C}, 84.19 \% ; \mathrm{H}, 8.64 \%$; N, 2.18\%. Found: C, 84.13\%; H, 8.69\%; N, $2.15 \%$. 


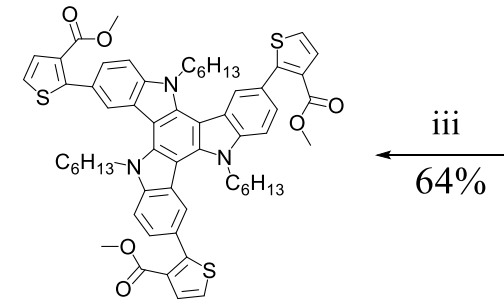

13

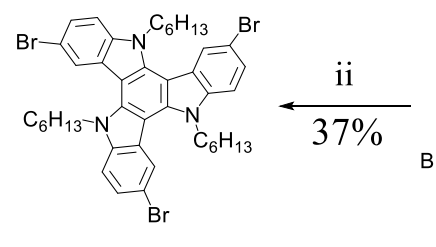

12

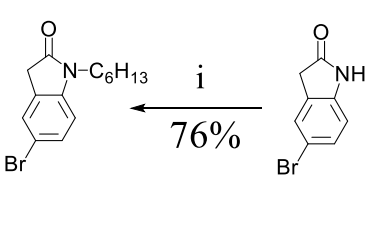

11

1

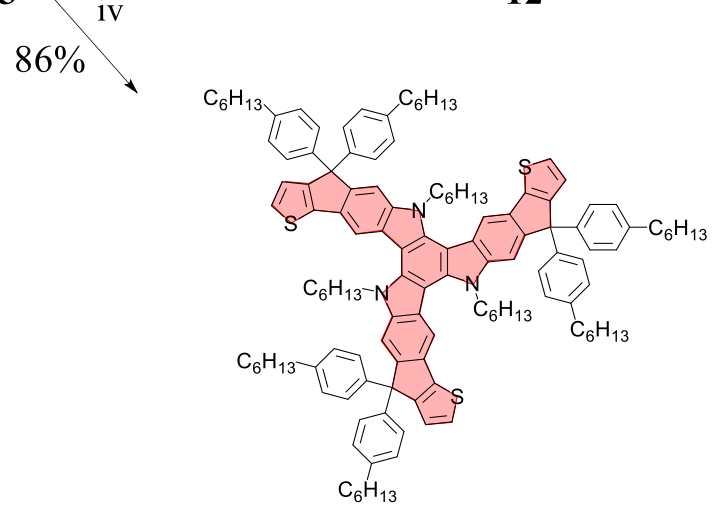

T1-C6

Scheme S1. Synthetic route for T1-C6 core. Reagents and conditions: (i) 1-bromohexane, $\mathrm{K}_{2} \mathrm{CO}_{3}$, 18-crown-6, AN, $80{ }^{\circ} \mathrm{C}, 24$ h; (ii) $\mathrm{POCl}_{3}, 100{ }^{\circ} \mathrm{C}, 20$ h; (iii) methyl thiophene-3-carboxylate, $\mathrm{Pd}(\mathrm{OAc})_{2}, \mathrm{PCy}_{3} \cdot \mathrm{HBF}_{4}, \mathrm{PivOH}, \mathrm{K}_{2} \mathrm{CO}_{3}$, toluene, reflux, $48 \mathrm{~h}$; (iv) (4-hexylphenyl)lithium, $-78^{\circ} \mathrm{C}$ to $\mathrm{rt}, 1 \mathrm{~h}$; Then boron trifluoride-diethyl etherate, dichloromethane, $0{ }^{\circ} \mathrm{C}, 10 \mathrm{~min}$.

\section{5-Bromo-1-hexylindolin-2-one (11)}

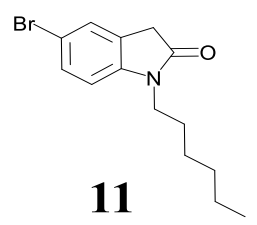

1 (5.00 g, $23.58 \mathrm{mmol}$ ), 1-bromohexane (4.28 g, $25.94 \mathrm{mmol}), \mathrm{K}_{2} \mathrm{CO}_{3}$ (3.58 g, $25.94 \mathrm{mmol}$ ) and 18-crown-6 (6.85 g, $25.94 \mathrm{mmol})$ were dissolved in acetonitrile $(100 \mathrm{~mL})$ in a three-neck roundbottom flask. The resulting mixture was stirred for $24 \mathrm{~h}$ at $80{ }^{\circ} \mathrm{C}$ and then cooled to room temperature. Dichloromethane $(80 \mathrm{~mL})$ was added and the organic layer was washed with water $(80 \mathrm{~mL} \times 3)$ and dried over anhydrous sodium sulfate. After removing solvent under reduced pressure, the residue was purified by column chromatography (ethyl acetate/petroleum ether 40- 
$\left.60{ }^{\circ} \mathrm{C}, 1 / 8, v / v\right)$ on silica gel to afford a colorless oil as the desired product $\mathbf{1 1}(5.30 \mathrm{~g}, 76 \%$ yield). The characterization of NMR and MS are the same with literature. ${ }^{[\mathrm{S} 6]}$ 3,8,13-Tribromo-5,10,15-trihexyl-10,15-dihydro-5H-diindolo[3,2-a:3',2'-c]carbazole (12)

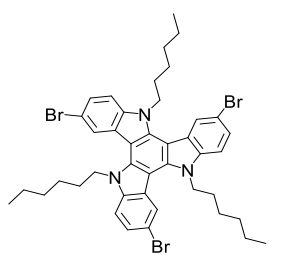

12

11 (5.00 g, $16.89 \mathrm{mmol})$ was dissloved in $\mathrm{POCl}_{3}(50 \mathrm{~mL})$ and the resulting mixture was stirred for $20 \mathrm{~h}$ at $100{ }^{\circ} \mathrm{C}$ and then cooled to room temperature. The excessive $\mathrm{POCl}_{3}$ was distilled under vacuum and the residue was meutralized by $\mathrm{KOH}$ aqueous solution. Dichloromethane (80 $\mathrm{mL})$ was added and the organic layer was washed with water $(50 \mathrm{~mL} \times 3)$ and dried over anhydrous sodium sulfate. After removing solvent under reduced pressure, the residue was purified by column chromatography (DCM/petroleum ether $\left.40-60{ }^{\circ} \mathrm{C}, 1 / 10, v / v\right)$ on silica gel to afford a white solid as the desired product 12 (1.74 g, 37\% yield). ${ }^{1} \mathrm{H}$ NMR (400 MHz, $\left.\mathrm{CDCl}_{3}\right) \delta: 7.44(\mathrm{~s}, 3 \mathrm{H}), 7.36$ $(\mathrm{d}, J=8.5 \mathrm{~Hz}, 3 \mathrm{H}), 7.28\left(\mathrm{dd}, J_{1}=8.4 \mathrm{~Hz}, J_{2}=1.3 \mathrm{~Hz}, 3 \mathrm{H}\right), 4.12(\mathrm{t}, J=7.5 \mathrm{~Hz}, 6 \mathrm{H}), 1.76-1.71(\mathrm{~m}$, $6 \mathrm{H}), 1.34$ (br, 18H), $0.93(\mathrm{t}, J=6.8 \mathrm{~Hz}, 9 \mathrm{H}) .{ }^{13} \mathrm{C} \mathrm{NMR}\left(125 \mathrm{MHz}, \mathrm{CDCl}_{3}\right) \delta: 136.17,125.92$, $124.02,120.19,116.58,114.98,112.62,93.17,46.16,31.44,29.82,26.43,22.59,14.09$.

Trimethyl 2,2',2"-(5,10,15-trihexyl-10,15-dihydro-5H-diindolo[3,2-a:3',2'-c]carbazole-3,8,13triyl)tris(thiophene-3-carboxylate) (13)

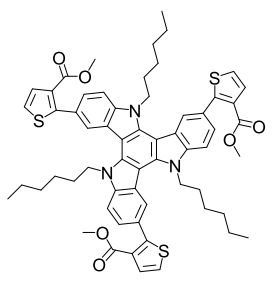

13

In a dried two-neck round-bottom flask were dissolved compound 12 (1.00 g, $1.20 \mathrm{mmol})$, methyl thiophene-3-carboxylate $(681 \mathrm{mg}, 4.80 \mathrm{mmol})$ and $\mathrm{K}_{2} \mathrm{CO}_{3}(1.64 \mathrm{~g}, 11.87 \mathrm{mmol})$ in toluene (30 $\mathrm{mL})$. Then $\mathrm{Pd}(\mathrm{OAc})_{2}(136 \mathrm{mg}, 0.48 \mathrm{mmol}), \mathrm{PCy}_{3} \cdot \mathrm{HBF}_{4}(342 \mathrm{mg}, 0.94 \mathrm{mmol})$, and PivOH (251 $\mathrm{mg}, 2.38 \mathrm{mmol}$ ) were added to the reaction mixture in a nitrogen-filled glovebox, which was 
refluxed for $48 \mathrm{~h}$. Then the resulting solution cooled down to room temperature and chloroform $(50 \mathrm{~mL})$ was added. The organic layer was washed with brine $(50 \mathrm{~mL} \times 3)$ and dried over anhydrous sodium sulfate. After solvent removal under reduced pressure, the crude product was purified by column chromatography (DCM/petroleum ether $\left.40-60^{\circ} \mathrm{C}, 4 / 1, v / v\right)$ on silica gel to yield a yellow solid as the desired product 13 (781 mg, 64\% yield). ${ }^{1} \mathrm{H}$ NMR (400 $\left.\mathrm{MHz}, \mathrm{CDCl}_{3}\right)$ $\delta: 8.25(\mathrm{~d}, J=8.4 \mathrm{~Hz}, 3 \mathrm{H}), 7.85(\mathrm{t}, J=4.3 \mathrm{~Hz}, 3 \mathrm{H}), 7.65-7.62(\mathrm{~m}, 3 \mathrm{H}), 7.55(\mathrm{~d}, J=8.2 \mathrm{~Hz}, 3 \mathrm{H})$, $7.32(\mathrm{~d}, J=5.4 \mathrm{~Hz}, 3 \mathrm{H}), 4.86(\mathrm{t}, J=7.4 \mathrm{~Hz}, 6 \mathrm{H}), 3.84(\mathrm{~s}, 9 \mathrm{H}), 2.08$ (br, 6H), 1.32-1.28 (m, 18H), 0.88-0.85 (m, 9H). ${ }^{13} \mathrm{C}$ NMR (125 MHz, $\left.\mathrm{CDCl}_{3}\right) \delta: 164.07,152.45,139.72,130.34,127.49$, $127.34,123.96,123.53,121.74,120.91,112.06,102.99,51.64,47.10,31.49,29.98,26.43,22.58$, 14.01 .

6,13,20-Trihexyl-4,4,11,11,18,18-hexakis(4-hexylphenyl)-4,6,11,13,18,20-

hexahydrothieno[3", 2":4',5']cyclopenta[1',2':5,6]indolo[3,2-

a]thieno[3",2": $\left.4^{\prime}, 5^{\prime}\right]$ cyclopenta[ $\left[1^{\prime}, 2^{\prime}: 5,6\right]$ indolo[3,2-c]thieno $\left[3^{\prime}, 2^{\prime}: 4,5\right]$ cyclopenta[1,2-

h]carbazole (T1-C6)

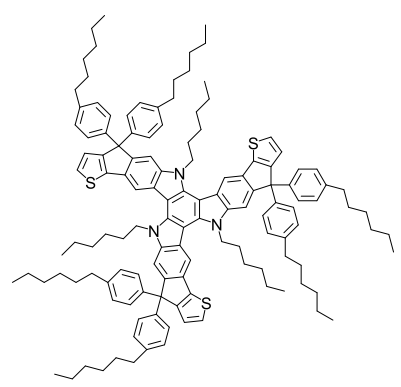

T1-C6

In a flame-dried two-neck flask were added $13(386 \mathrm{mg}, 0.36 \mathrm{mmol})$ and $30 \mathrm{~mL}$ anhydrous THF and the mixture was cooled to $0^{\circ} \mathrm{C}$ using ice-salt baths. Then (4-hexylphenyl)lithium ( $3 \mathrm{~mL}, 1 \mathrm{M}$ in THF, $3.00 \mathrm{mmol}$ ) was injected one portion via syringe before the resulting solution was heated to room temperature. The mixture was further stirred at room temperature for $1 \mathrm{~h}$ and then $1 \mathrm{M}$ $\mathrm{HCl}$ aqueous solution $(50 \mathrm{~mL})$ was added to terminate the reaction. Chloroform $(60 \mathrm{~mL})$ was added and the organic layer was washed with brine $(50 \mathrm{~mL} \times 3)$ and dried over anhydrous sodium sulfate. After desiccant removal by filtering, the filtrate was concentrated under reduced pressure. The crude product was used in the subsequent reaction directly. In a flame-dried two-neck flask, the above tertiary alcohol was added into $30 \mathrm{~mL}$ anhydrous DCM. The resulting mixture was cooled 
to $0{ }^{\circ} \mathrm{C}$ before $0.2 \mathrm{~mL}$ boron trifluoride-diethyl etherate was added. After stirred at $0{ }^{\circ} \mathrm{C}$ for $10 \mathrm{~min}$, the solution was quenched by $1 \mathrm{M} \mathrm{K}_{2} \mathrm{CO}_{3}$ aqueous solution $(60 \mathrm{~mL})$. Then the mixture was extracted three times with chloroform $(60 \mathrm{~mL} \times 3)$ before the organic phase was washed with water and dried over anhydrous sodium sulfate. After solvent removal under reduced pressure, the crude product was purified by column chromatography (DCM/petroleum ether $\left.40-60{ }^{\circ} \mathrm{C}, 1 / 10, v / v\right)$ on silica gel to yield a yellow solid as the desired product T1-C6 (569 mg, 86\% yield). ${ }^{1} \mathrm{H}$ NMR (500 $\left.\mathrm{MHz} \mathrm{CDCl}_{3}\right) \delta: 8.17(\mathrm{~s}, 3 \mathrm{H}), 7.60(\mathrm{~s}, 3 \mathrm{H}), 7.28(\mathrm{~d}, J=8.1 \mathrm{~Hz}, 15 \mathrm{H}), 7.09(\mathrm{~d}, J=5.1 \mathrm{~Hz}, 3 \mathrm{H})$, $7.07(\mathrm{~d}, J=8.2 \mathrm{~Hz}, 12 \mathrm{H}), 4.77(\mathrm{t}, J=7.1 \mathrm{~Hz}, 6 \mathrm{H}), 2.55(\mathrm{t}, J=7.6 \mathrm{~Hz}, 12 \mathrm{H}), 1.67$ (br, 6H), $1.61-$ $1.55(\mathrm{~m}, 12 \mathrm{H}), 1.34-1.28(\mathrm{~m}, 36 \mathrm{H}), 1.01-0.97(\mathrm{~m}, 18 \mathrm{H}), 0.86(\mathrm{t}, J=6.5 \mathrm{~Hz}, 18 \mathrm{H}), 0.69(\mathrm{t}, J=6.7$ $\mathrm{Hz}, 9 \mathrm{H}) .{ }^{13} \mathrm{C} \mathrm{NMR}\left(125 \mathrm{MHz}, \mathrm{CDCl}_{3}\right) \delta: 156.25,146.43,143.27,141.85,141.28,141.08,138.42$, 132.82, 128.37, 127.98, 127.13, 123.45, 121.77, 119.97, 104.43, 101.53, 62.68, 47.17, 35.73, 31.87, $31.54,31.50,29.33,26.31,22.74,22.45,14.23,14.08$. 

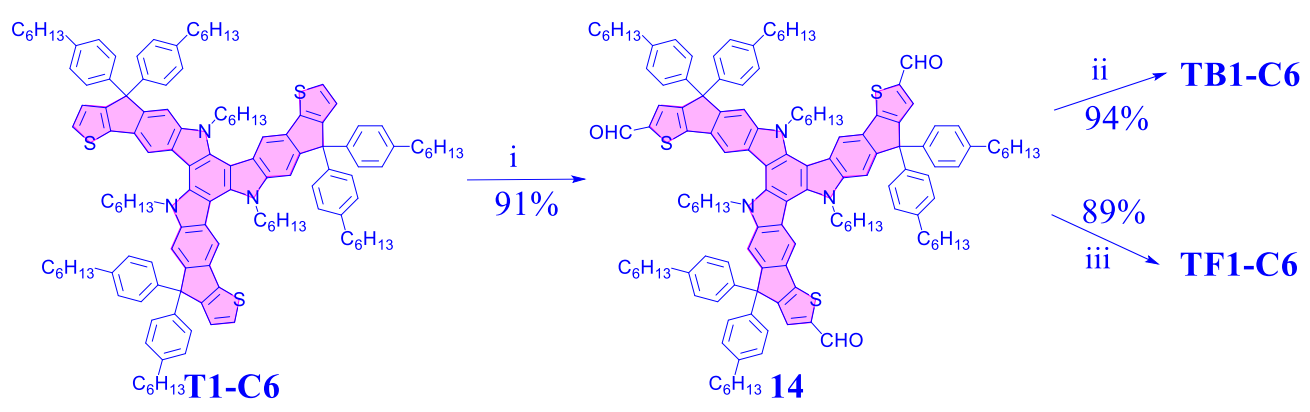

Scheme S2. Synthetic route to novel electron acceptors. Reagents and conditions: (i) $\mathrm{POCl}_{3}, \mathrm{DMF}$, reflux, 6 h; Then sodium acetate, RT, 2 h; (ii) 1,3-diethyl-2-thioxodihydropyrimidine-4,6(1H,5H)dione, $\beta$-alanine, DCE/ethanol (3/1, $v / v)$, reflux, overnight; (iii) 2-(5,6-difluoro-3-oxo-2,3dihydro- $1 H$-inden-1-ylidene)malononitrile, $\beta$-alanine, DCE/ethanol $(3 / 1, v / v)$, reflux, overnight. 
6,13,20-Trihexyl-4,4,11,11,18,18-hexakis(4-hexylphenyl)-4,6,11,13,18,20-

hexahydrothieno $\left[3^{\prime \prime}, 2^{\prime \prime}: 4^{\prime}, 5^{\prime}\right]$ cyclopenta $\left[1^{\prime}, 2^{\prime}: 5,6\right]$ indolo[3,2-

a]thieno[3", $\left.2^{\prime \prime}: 4^{\prime}, 5^{\prime}\right]$ cyclopenta[ $\left[1^{\prime}, 2^{\prime}: 5,6\right]$ indolo[3,2-c]thieno $\left[3^{\prime}, 2^{\prime}: 4,5\right]$ cyclopenta $[1,2$ -

h]carbazole-2,9,16-tricarbaldehyde (14)

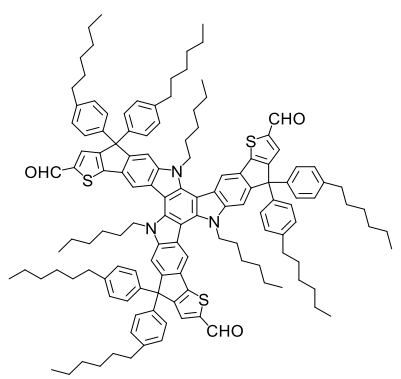

14

In a dry round-bottom flask were added T1-C6 $(387 \mathrm{mg}, 0.21 \mathrm{mmol})$ and $30 \mathrm{~mL}$ anhydrous dichloroethane. Then DMF $(5 \mathrm{~mL})$ and $\mathrm{POCl}_{3}(1 \mathrm{~mL})$ were added and the reaction solution was stirred at reflux for $6 \mathrm{~h}$. Saturated sodium acetate aqueous solution (20 mL) was added and the mixture was stirred for another $2 \mathrm{~h}$. Then the mixture was extracted into dichloromethane $(50 \mathrm{~mL})$, and the organic layer was washed with water $(50 \mathrm{~mL} \times 3)$ and dried over anhydrous sodium sulfate. After removing solvent under reduced pressure, the residue was purified by column chromatography (DCM) on silica gel to yield an orange solid as the desired product 14 (368 $\mathrm{mg}$, 91\% yield). ${ }^{1} \mathrm{H}$ NMR (500 MHz, $\left.\mathrm{CDCl}_{3}\right) \delta: 9.87$ (s, 3H), $8.16(\mathrm{~s}, 3 \mathrm{H}), 7.73$ (d, J=4.4 Hz, 6H), $7.24(\mathrm{~d}, J=8.1 \mathrm{~Hz}, 12 \mathrm{H}), 7.09(\mathrm{~d}, J=8.1 \mathrm{~Hz}, 12 \mathrm{H}), 4.80(\mathrm{t}, J=7.3 \mathrm{~Hz}, 6 \mathrm{H}), 2.59(\mathrm{t}, J=7.6 \mathrm{~Hz}$, $12 \mathrm{H}), 1.68(\mathrm{t}, J=6.1 \mathrm{~Hz}, 6 \mathrm{H}), 1.65-1.57(\mathrm{~m}, 12 \mathrm{H}), 1.37-1.34(\mathrm{~m}, 12 \mathrm{H}), 1.32-1.30(\mathrm{~m}, 24 \mathrm{H})$, $1.02-0.97(\mathrm{~m}, 18 \mathrm{H}), 0.89(\mathrm{t}, J=6.3 \mathrm{~Hz}, 18 \mathrm{H}), 0.70(\mathrm{t}, J=6.4 \mathrm{~Hz}, 9 \mathrm{H}) .{ }^{13} \mathrm{C} \mathrm{NMR}(125 \mathrm{MHz}$, $\left.\mathrm{CDCl}_{3}\right) \delta: 182.81,156.50,151.96,147.53,145.27,141.96,141.86,140.96,139.60,132.50,131.01$, 128.52, 127.64, 123.71, 119.76, 104.24, 103.19, 62.74, 47.20, 35.56, 31.71, 31.40, 31.27, 29.23, $29.16,26.10,22.60,22.26,14.08,13.90$.

5,5',5"-((6,13,20-Trihexyl-4,4,11,11,18,18-hexakis(4-hexylphenyl)-4,6,11,13,18,20hexahydrothieno[3", 2":4',5']cyclopenta[1',2':5,6]indolo[3,2a]thieno[3",2": $\left.4^{\prime}, 5^{\prime}\right]$ cyclopenta[1',2':5,6]indolo[3,2-c]thieno $\left[3^{\prime}, 2 ': 4,5\right]$ cyclopenta[1,2h]carbazole-2,9,16-triyl)tris(methaneylylidene))tris(1,3-diethyl-2-thioxodihydropyrimidine4,6(1H,5H)-dione) (TB1-C6) 


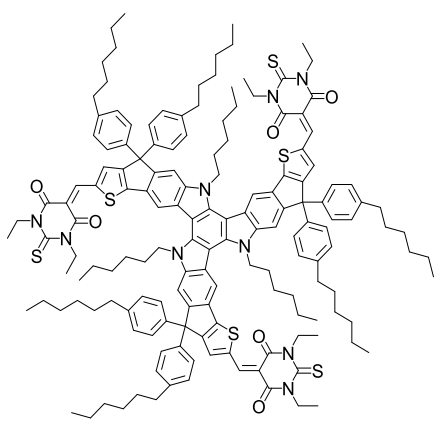

TB1-C6

Compound 14 (96 mg, 0.05 mmol), 1,3-diethyl-2-thioxodihydropyrimidine-4,6(1H,5H)-dione (60 $\mathrm{mg}, 0.30 \mathrm{mmol})$ and $\beta$-alanine $(2 \mathrm{mg}$ ) were dissolved in the mixture of dichloroethane and ethanol $(20 \mathrm{~mL}, v / v, 3 / 1)$ in a two-neck round-bottom flask. Then the resultant mixture was stirred at reflux overnight before $80 \mathrm{~mL}$ methanol was added. The mixture was filtered and the residue was recrystallized in chloroform and methanol $(1 / 5, v / v)$ to obtain a brown solid as the desired product TB1-C6 (116 mg, 94\% yield). ${ }^{1} \mathrm{H}$ NMR (500 MHz, $\left.\mathrm{CDCl}_{3}\right) \delta: 8.74$ (s, 3H), 8.19 (s, 3H), 7.91 (s, $3 \mathrm{H}), 7.86(\mathrm{~s}, 3 \mathrm{H}), 7.29(\mathrm{~d}, J=7.8 \mathrm{~Hz}, 12 \mathrm{H}), 7.16(\mathrm{~d}, J=7.8 \mathrm{~Hz}, 12 \mathrm{H}), 4.75-4.71(\mathrm{~m}, 12 \mathrm{H}), 4.62$ $(\mathrm{d}, J=6.9 \mathrm{~Hz}, 6 \mathrm{H}), 2.62(\mathrm{t}, J=7.8 \mathrm{~Hz}, 12 \mathrm{H}), 1.75(\mathrm{br}, 6 \mathrm{H}), 1.68-1.60(\mathrm{~m}, 12 \mathrm{H}), 1.45(\mathrm{t}, J=6.9$ $\mathrm{Hz}, 9 \mathrm{H}), 1.40-1.32(\mathrm{~m}, 45 \mathrm{H}), 1.07-1.03(\mathrm{~m}, 18 \mathrm{H}), 0.90(\mathrm{t}, J=6.6 \mathrm{~Hz}, 18 \mathrm{H}), 0.75$ (t, $J=6.8 \mathrm{~Hz}$, 9H). $\left.{ }^{13} \mathrm{C} \mathrm{NMR} \mathrm{(125} \mathrm{MHz,} \mathrm{CDCl}_{3}\right) \delta: 178.58,162.50,161.13,160.30,157.54,149.67,148.78$, 142.09 , 141.77, 141.29, 141.09, 140.58, 140.54, 131.20, 128.62, 127.66, 125.04, 119.71, 108.43, 104.27, 62.71, 47.40, 43.95, 43.20, 41.07, 39.13, 35.59, 31.72, 31.40, 29.17, 26.27, 22.62, 22.38, $14.10,13.95,12.61,12.41$.

2,2',2"-((2Z,2'Z,2'Z)-((6,13,20-trihexyl-4,4,11,11,18,18-hexakis(4-hexylphenyl)-4,6,11,13,18,20hexahydrothieno[3",2":4',5']cyclopenta[1',2':5,6]indolo[3,2-

a]thieno[3",2": $\left.4^{\prime}, 5^{\prime}\right]$ cyclopenta[ $\left.1^{\prime}, 2^{\prime}: 5,6\right]$ indolo[3,2-c]thieno $\left[3^{\prime}, 2 ': 4,5\right]$ cyclopenta[1,2h]carbazole-2,9,16-triyl)tris(methaneylylidene))tris(5,6-difluoro-3-oxo-2,3-dihydro-1H-indene2,1-diylidene))trimalononitrile (TF1-C6) 


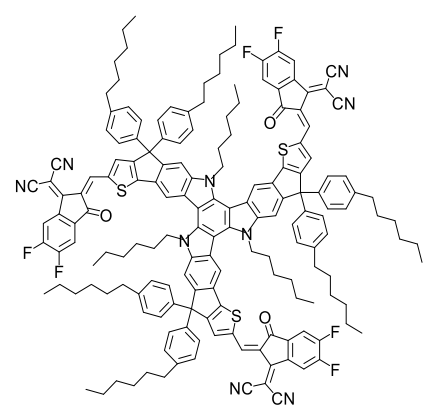

TF1-C6

Compound 14 (193 mg, 0.10 mmol), 2-(5,6-difluoro-3-oxo-2,3-dihydro-1H-inden-1ylidene)malononitrile (138 $\mathrm{mg}, 0.60 \mathrm{mmol})$ and $\beta$-alanine $(2 \mathrm{mg})$ were dissolved in the mixture of dichloroethane and ethanol $(20 \mathrm{~mL}, v / v, 3 / 1)$ in a two-neck round-bottom flask. Then the resultant mixture was stirred at reflux overnight. Then the solvent was removed under reduced pressure and the crude product was purified by column chromatography (DCM) on silica gel to afford a brown powder as the desired product TF1-C6 (228 mg, 89\% yield). ${ }^{1} \mathrm{H}$ NMR (500 MHz, $\left.\mathrm{CDCl}_{3}\right) \delta: 8.67$ $(\mathrm{s}, 3 \mathrm{H}), 8.43\left(\mathrm{dd}, J_{1}=9.3 \mathrm{~Hz}, J_{2}=2.5 \mathrm{~Hz}, 3 \mathrm{H}\right), 8.34(\mathrm{~d}, J=7.9 \mathrm{~Hz}, 6 \mathrm{H}), 8.04(\mathrm{~s}, 3 \mathrm{H}), 7.99(\mathrm{~s}, 3 \mathrm{H})$, $7.80(\mathrm{~s}, 3 \mathrm{H}), 7.38(\mathrm{~d}, J=8.1 \mathrm{~Hz}, 6 \mathrm{H}), 7.15-7.10(\mathrm{~m}, 12 \mathrm{H}), 6.50(\mathrm{~s}, 3 \mathrm{H}), 4.26-4.23(\mathrm{~m}, 3 \mathrm{H}), 2.71-$ $2.57(\mathrm{~m}, 12 \mathrm{H}), 2.44-2.40(\mathrm{~m}, 3 \mathrm{H}), 1.81-1.72(\mathrm{~m}, 6 \mathrm{H}), 1.62-1.58(\mathrm{~m}, 6 \mathrm{H}), 1.51-1.50(\mathrm{~m}, 16 \mathrm{H})$, 1.36-1.29 (m, 22H), 0.98 (br, 8H), 0.86 (br, 8H), 0.71 (br, 6H), 0.56-0.53 (m, 6H), 0.46-0.43 (m, $6 \mathrm{H}), 0.28$ (br, 9H), 0.05 (br, 3H), -0.14 (br, 3H). $\left.{ }^{13} \mathrm{C} \mathrm{NMR} \mathrm{(125} \mathrm{MHz,} \mathrm{CDCl}_{3}\right) \delta: 185.52,163.22$, 156.83, 156.67, 155.65, 153.56, 150.10, 147.03, 143.37, 142.97, 142.90, 142.08, 141.59, 141.14, $140.21,138.57,138.06,134.27,131.32,129.35,129.06,128.69,127.90,127.66,123.90,118.99$, 118.37, 114.93, 114.75, 114.17, 113.93, 113.33, 113.17, 104.66, 104.56, 66.78, 63.06, 45.61, 35.81, $35.59,32.19,31.66,31.48,31.39,31.09,30.78,29.35,29.17,28.26,26.19,22.78,22.59,21.83$, $14.21,14.03,13.45$.

6,13,20-Tris(2-ethylhexyl)-4,4,11,11,18,18-hexakis(4-hexylphenyl)-4,6,11,13,18,20-

hexahydrothieno[3",2":4',5']cyclopenta[1',2':5,6]indolo[3,2 -

a]thieno[3",2": $\left.4^{\prime}, 5^{\prime}\right]$ cyclopenta[ $\left.1^{\prime}, 2^{\prime}: 5,6\right]$ indolo[3,2-c]thieno $\left[3^{\prime}, 2^{\prime}: 4,5\right]$ cyclopenta[1,2h]carbazole-2,9,16-tricarbaldehyde (15) 


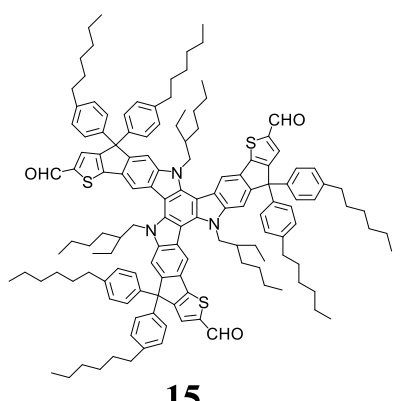

15

In a dry round-bottom flask were added T1 $(400 \mathrm{mg}, 0.21 \mathrm{mmol})$ and $30 \mathrm{~mL}$ anhydrous dichloroethane. Then DMF $(5 \mathrm{~mL})$ and $\mathrm{POCl}_{3}(1 \mathrm{~mL})$ were added and the reaction solution was stirred at reflux for $6 \mathrm{~h}$. Saturated sodium acetate aqueous solution (20 mL) was added and the mixture was stirred for another $2 \mathrm{~h}$. Then the mixture was extracted into dichloromethane $(50 \mathrm{~mL})$, and the organic layer was washed with water $(50 \mathrm{~mL} \times 3)$ and dried over anhydrous sodium sulfate. After removing solvent under reduced pressure, the residue was purified by column chromatography (DCM) on silica gel to yield an orange solid as the desired product 15 (392 $\mathrm{mg}$, 93\% yield). ${ }^{1} \mathrm{H}$ NMR (400 MHz, $\left.\mathrm{CDCl}_{3}\right) \delta$ : 9.92 (s, 3H), 8.26 (s, 3H), 7.81 (br, 6H), 7.38-7.28 (m, 12H), 7.23-7.11 (m, 12H), 4.80 (br, 6H), 2.63 (d, $J=6.8 \mathrm{~Hz}, 12 \mathrm{H}), 2.00$ (br, 3H), 1.68-1.62 (m, $12 \mathrm{H}), 1.37-1.35(\mathrm{~m}, 36 \mathrm{H}), 0.99-0.92(\mathrm{~m}, 30 \mathrm{H}), 0.74-0.63(\mathrm{~m}, 21 \mathrm{H}), 0.47-0.37(\mathrm{~m}, 9 \mathrm{H}) .{ }^{13} \mathrm{C} \mathrm{NMR}$ $\left(125 \mathrm{MHz}, \mathrm{CDCl}_{3}\right) \delta: 182.8120 .18,2,156.55,152.04,147.40,145.29,142.17,142.12,141.78$, $141.00,140.90,140.23$, 132.56, 130.88, 128.58, 127.76, 127.67, 123.82, 104.32, 104.05, 62.78, 50.72, 50.62, 37.85, 37.71, 35.64, 31.78, 31.44, 31.38, 29.20, 28.17, 28.02, 27.95, 27.78. HR-MS (MALDI-TOF) $\mathrm{m} / z$ calcd. for $\left(\mathrm{C}_{138} \mathrm{H}_{165} \mathrm{~N}_{3} \mathrm{O}_{3} \mathrm{~S}_{3}\right)$ : 2009.20446. Found: 2009.20486. Anal. Calcd. for $\mathrm{C}_{138} \mathrm{H}_{165} \mathrm{~N}_{3} \mathrm{O}_{3} \mathrm{~S}_{3}: \mathrm{C}, 82.46 \% ; \mathrm{H}, 8.27 \% ; \mathrm{N}, 2.09 \%$. Found: C, 82.41\%; H, 8.32\%; N, $2.07 \%$. 5,5',5"-((6,13,20-Tris(2-ethylhexyl)-4,4,11,11,18,18-hexakis(4-hexylphenyl)-4,6,11,13,18,20hexahydrothieno[3",2":4',5']cyclopenta[1',2':5,6]indolo[3,2a]thieno[3",2": $\left.4^{\prime}, 5^{\prime}\right]$ cyclopenta[ $\left.1^{\prime}, 2^{\prime}: 5,6\right]$ indolo[3,2-c]thieno $\left[3^{\prime}, 2^{\prime}: 4,5\right]$ cyclopenta[1,2h]carbazole-2,9,16-triyl)tris(methanylylidene))tris(1,3-diethyl-2-thioxodihydropyrimidine4,6(1H,5H)-dione) (TB1) 


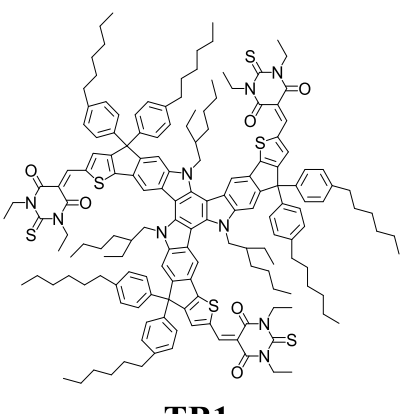

TB1

Compound 15 (100 mg, $0.05 \mathrm{mmol})$, 1,3-diethyl-2-thioxodihydropyrimidine-4,6(1H,5H)-dione $(60 \mathrm{mg}, 0.30 \mathrm{mmol})$ and $\beta$-alanine $(2 \mathrm{mg})$ were dissolved in the mixture of dichloroethane and ethanol $(20 \mathrm{~mL}, v / v, 3 / 1)$ in a two-neck round-bottom flask. Then the resultant mixture was stirred at reflux overnight before $80 \mathrm{~mL}$ methanol was added. The mixture was filtered and the residue was recrystallized in chloroform and methanol $(1 / 5, v / v)$ to obtain a brown solid as the desired product TB1 (120 mg, 94\% yield). ${ }^{1} \mathrm{H}$ NMR (500 MHz, $\left.\mathrm{CDCl}_{3}\right) \delta: 8.71$ (s, 3H), 8.19 (s, 3H), 7.89 (s, 3H), $7.83(\mathrm{~s}, 3 \mathrm{H}), 7.30-7.22(\mathrm{~m}, 12 \mathrm{H}), 7.15-7.09(\mathrm{~m}, 12 \mathrm{H}), 4.73-4.67(\mathrm{~m}, 12 \mathrm{H}), 4.60(\mathrm{~d}, J=$ $6.8 \mathrm{~Hz}, 6 \mathrm{H}), 2.60-2.58(\mathrm{~m}, 12 \mathrm{H}), 1.98(\mathrm{br}, 3 \mathrm{H}), 1.63-1.59(\mathrm{~m}, 12 \mathrm{H}), 1.43$ (t. $J=6.9 \mathrm{~Hz}, 9 \mathrm{H})$, $1.33-1.31(\mathrm{~m}, 36 \mathrm{H}), 0.89-0.88(\mathrm{~m}, 30 \mathrm{H}), 0.61-0.56(\mathrm{~m}, 21 \mathrm{H}), 0.44-0.35(\mathrm{~m}, 9 \mathrm{H}) .{ }^{13} \mathrm{C}$ NMR $(125$ $\left.\mathrm{MHz}_{\mathrm{CDCl}}\right) \delta: 178.59,162.55,161.14,160.29,157.55,149.67,148.61,141.98,141.91,141.87$, 141.12 , 140.53, 131.05, 128.61, 127.72, 127.62, 125.06, 120.13, 108.41, 105.08, 104.46, 62.66, 50.90, 43.94, 43.19, 37.58, 35.59, 31.73, 31.38, 31.33, 29.20, 28.92, 27.89, 27.82, 27.69, 27.61, 22.61, 14.11, 13.90, 13.88, 12.63, 12.41, 9.92, 9.83, 9.61, 9.42. HR-MS (MALDI-TOF) $\mathrm{m} / \mathrm{z}$ calcd. for $\left(\mathrm{C}_{162} \mathrm{H}_{195} \mathrm{~N}_{9} \mathrm{O}_{6} \mathrm{~S}_{6}\right)$ : 2555.35881. Found: 2555.35901. Anal. Calcd. for $\mathrm{C}_{162} \mathrm{H}_{195} \mathrm{~N}_{9} \mathrm{O}_{6} \mathrm{~S}_{6}$ : C, 76.10\%; H, 7.69\%; N, 4.93\%. Found: C, 76.07\%; H, 7.72\%; N, 4.91\%. Solubility in chlorobenzene: $\sim 58 \mathrm{mg} / \mathrm{mL}$.

2,2',2"-((2Z,2'Z,2'Z)-((6,13,20-Tris(2-ethylhexyl)-4,4,11,11,18,18-hexakis(4-hexylphenyl)-

4,6,11,13,18,20-hexahydrothieno[3",2":4',5']cyclopenta[1',2':5,6]indolo[3,2-

a]thieno[3",2": $\left.4^{\prime}, 5^{\prime}\right]$ cyclopenta[ $\left.1^{\prime}, 2^{\prime}: 5,6\right]$ indolo[3,2-c]thieno[3',2':4,5]cyclopenta[1,2h]carbazole-2,9,16-triyl)tris(methanylylidene))tris(5,6-difluoro-3-oxo-2,3-dihydro-1H-indene2,1-diylidene))trimalononitrile (TF 1) 


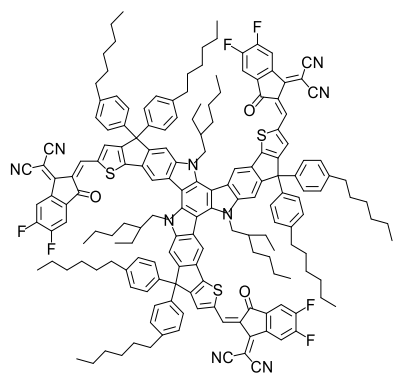

TF1

Compound 15 (200 mg, 0.10 mmol), 2-(5,6-difluoro-3-oxo-2,3-dihydro-1H-inden-1ylidene)malononitrile (138 $\mathrm{mg}, 0.60 \mathrm{mmol})$ and $\beta$-alanine $(2 \mathrm{mg}$ ) were dissolved in the mixture of dichloroethane and ethanol $(20 \mathrm{~mL}, v / v, 3 / 1)$ in a two-neck round-bottom flask. Then the resultant mixture was stirred at reflux overnight. Then the solvent was removed under reduced pressure and the crude product was purified by column chromatography (DCM) on silica gel to afford a brown powder as the desired product TF1 (135 mg, 51\% yield). ${ }^{1} \mathrm{H}$ NMR (400 $\left.\mathrm{MHz}, \mathrm{CDCl}_{3}\right) \delta: 8.94$ (s, $3 \mathrm{H}), 8.56\left(\mathrm{dd}, J_{1}=9.8 \mathrm{~Hz}, J_{2}=3.3 \mathrm{~Hz}, 3 \mathrm{H}\right), 8.19(\mathrm{~s}, 3 \mathrm{H}), 7.89(\mathrm{~s}, 3 \mathrm{H}), 7.83(\mathrm{~s}, 3 \mathrm{H}), 7.72(\mathrm{t}, J=$ $7.5 \mathrm{~Hz}, 3 \mathrm{H}), 7.24-7.18(\mathrm{~m}, 12 \mathrm{H}), 7.16-7.10(\mathrm{~m}, 12 \mathrm{H}), 4.76-4.70(\mathrm{~m}, 6 \mathrm{H}), 2.60(\mathrm{~d}, J=7.2 \mathrm{~Hz}$, $12 \mathrm{H}), 2.00(\mathrm{br}, 3 \mathrm{H}), 1.65-1.58(\mathrm{~m}, 12 \mathrm{H}), 1.35-1.27(\mathrm{~m}, 36 \mathrm{H}), 0.89-0.56(\mathrm{~m}, 51 \mathrm{H}), 0.50-0.36(\mathrm{~m}$, 9H). $\left.{ }^{13} \mathrm{C} \mathrm{NMR} \mathrm{(125} \mathrm{MHz,} \mathrm{CDCl}_{3}\right) \delta: 186.24,162.79,158.45,158.16,155.49,155.37,153.39$, 153.31, 149.07, 142.11, 141.57, 141.45, 141.19, 140.56, 140.31, 138.67, 136.58, 134.53, 130.89, 128.70, 127.75, 127.64, 125.37, 120.14, 115.10, 114.93, 114.54, 114.48, 112.58, 112.41, 105.12, 104.62, 104.54, 68.75, 62.70, 50.91, 37.78, 37.67, 35.59, 31.74, 31.72, 31.38, 31.34, 29.72, 29.17, 29.07, 29.02, 27.92, 27.83, 27.72, 27.62, 22.62, 22.60, 14.11, 13.92, 13.89, 13.84, 9.92, 9.80, 9.58, 9.48. H-F decoupled ${ }^{19} \mathrm{~F}$ NMR (376 MHz, $\left.\mathrm{CDCl}_{3}\right): \delta:-123.12(\mathrm{~d}, J=19.3 \mathrm{~Hz}, 3 \mathrm{~F}), 124.30(\mathrm{~d}, J$ $=19.3 \mathrm{~Hz}, 3 \mathrm{~F})$. H-F coupled ${ }^{19} \mathrm{~F}$ NMR (376 MHz, $\left.\mathrm{CDCl}_{3}\right): \delta:-123.07 \sim-123.14(\mathrm{~m}, 3 \mathrm{~F}),-124.25$ $\sim-124.32$ (m, 3F). HR-MS (MALDI-TOF) $m / z$ calcd. for $\left(\mathrm{C}_{174} \mathrm{H}_{171} \mathrm{~F}_{6} \mathrm{~N}_{9} \mathrm{O}_{3} \mathrm{~S}_{3}\right)$ : 2645.26048. Found: 2645.26089. Anal. Calcd. for $\mathrm{C}_{174} \mathrm{H}_{171} \mathrm{~F}_{6} \mathrm{~N}_{9} \mathrm{O}_{3} \mathrm{~S}_{3}$ : C, 78.97\%; H, 6.51\%; N, 4.76\%. Found: C, $78.95 \%$; H, 6.54\%; N, 4.75\%. Solubility in chlorobenzene: $47 \mathrm{mg} / \mathrm{mL}$.

6,13,20-Tris(2-ethylhexyl)-4,4,11,11,18,18-hexakis(4-hexylphenyl)-4,6,11,13,18,20-

hexahydrothieno[2", $\left.3^{\prime \prime}: 3^{\prime}, 4^{\prime}\right]$ cyclopenta $\left[1^{\prime}, 2 ': 5,6\right]$ indolo[3,2 -

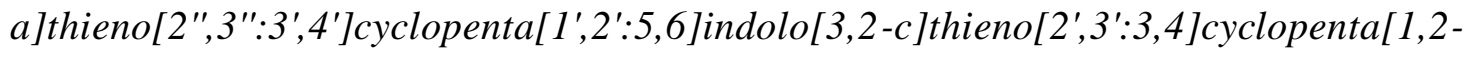

h]carbazole-2,9,16-tricarbaldehyde (16) 


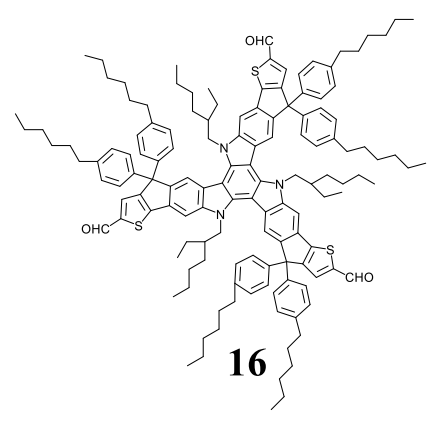

In a dry round-bottom flask were added T2 $(500 \mathrm{mg}, 0.25 \mathrm{mmol})$ and $30 \mathrm{~mL}$ anhydrous dichloroethane. Then DMF $(5 \mathrm{~mL})$ and $\mathrm{POCl}_{3}(1.5 \mathrm{~mL})$ were added and the reaction solution was stirred at reflux for $6 \mathrm{~h}$. Saturated sodium acetate aqueous solution $(20 \mathrm{~mL})$ was added and the mixture was stirred for another $2 \mathrm{~h}$. Then the mixture was extracted into dichloromethane $(50 \mathrm{~mL})$, and the organic layer was washed with water $(50 \mathrm{~mL} \times 3)$ and dried over anhydrous sodium sulfate. After removing solvent under reduced pressure, the residue was purified by column chromatography (DCM) on silica gel to yield an orange solid as the desired product 16 (452 $\mathrm{mg}$, 90\% yield). ${ }^{1} \mathrm{H}$ NMR (400 MHz, $\left.\mathrm{CDCl}_{3}\right) \delta: 9.91$ (s, 3H), 8.25 (s, 3H), 7.80 (br, 6H), 7.37-7.25 (m, 12H), 7.18-7.10 (m, 12H), 4.81-4.79 (m, 6H), 2.62 (d, $J=8.4 \mathrm{~Hz}, 12 \mathrm{H}), 2.00$ (br, 3H), 1.67-1.61 $(\mathrm{m}, 12 \mathrm{H}), 1.36-1.35(\mathrm{~m}, 36 \mathrm{H}), 0.98-0.92(\mathrm{~m}, 30 \mathrm{H}), 0.71-0.62(\mathrm{~m}, 21 \mathrm{H}), 0.52-0.36(\mathrm{~m}, 9 \mathrm{H}) .{ }^{13} \mathrm{C}$ NMR (125 MHz, $\left.\mathrm{CDCl}_{3}\right) \delta:$ 182.82, 156.55, 152.04, 147.40, 145.28, 142.15, 141.79, 140.99, $140.89,140.23,132.55,130.87,128.57,127.75,127.66,123.80,120.19$, 104.31, 104.03, 62.71, 50.71, 50.61, 37.85, 37.71, 35.63, 31.75, 31.36, 29.19, 22.63, 14.21, 13.99, 13.88, 9.93, 9.68, 9.52, 9.44. HR-MS (MALDI-TOF) $\mathrm{m} / z$ calcd. for $\left(\mathrm{C}_{138} \mathrm{H}_{165} \mathrm{~N}_{3} \mathrm{O}_{3} \mathrm{~S}_{3}\right)$ : 2009.20446. Found: 2009.20468. Anal. Calcd. for $\mathrm{C}_{138} \mathrm{H}_{165} \mathrm{~N}_{3} \mathrm{O}_{3} \mathrm{~S}_{3}: \mathrm{C}, 82.46 \% ; \mathrm{H}, 8.27 \%$; N, 2.09\%. Found: C, 82.44\%; H, 8.31\%; $\mathrm{N}, 2.09 \%$.

5,5',5"-((6,13,20-Tris(2-ethylhexyl)-4,4,11,11,18,18-hexakis(4-hexylphenyl)-4,6,11,13,18,20hexahydrothieno[2", $\left.3^{\prime \prime}: 3^{\prime}, 4^{\prime}\right]$ cyclopenta $\left[1^{\prime}, 2\right.$ ':5,6]indolo[3,2a]thieno[2",3": $\left.3^{\prime}, 4^{\prime}\right]$ cyclopenta[ $\left.1^{\prime}, 2^{\prime}: 5,6\right]$ indolo[3,2-c]thieno[ $\left.2^{\prime}, 3^{\prime}: 3,4\right]$ cyclopenta[1,2h]carbazole-2,9,16-triyl)tris(methanylylidene))tris(1,3-diethyl-2-thioxodihydropyrimidine4,6(1H,5H)-dione) ( $\mathbf{T B 2})$ 


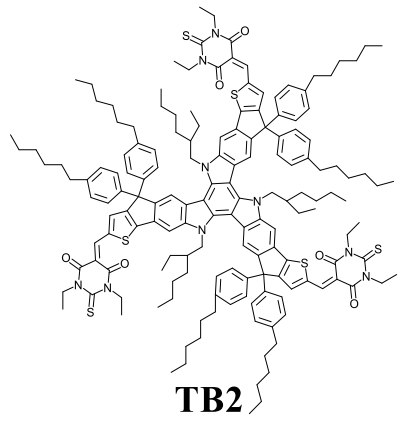

Compound 16 (100 mg, $0.05 \mathrm{mmol})$, 1,3-diethyl-2-thioxodihydropyrimidine-4,6(1H,5H)-dione $(60 \mathrm{mg}, 0.30 \mathrm{mmol})$ and $\beta$-alanine $(2 \mathrm{mg})$ were dissolved in the mixture of dichloroethane and ethanol $(20 \mathrm{~mL}, v / v, 3 / 1)$ in a two-neck round-bottom flask. Then the resultant mixture was stirred at reflux overnight before $80 \mathrm{~mL}$ methanol was added. The mixture was filtered and the residue was recrystallized in chloroform and methanol $(1 / 5, v / v)$ to obtain a brown solid as the desired product TB2 (117 mg, 92\% yield). ${ }^{1} \mathrm{H}$ NMR (500 MHz, $\left.\mathrm{CDCl}_{3}\right) \delta: 8.61$ (s, 3H), 8.09 (s, 3H), 7.79 $(\mathrm{s}, 3 \mathrm{H}), 7.73(\mathrm{~s}, 3 \mathrm{H}), 7.20-7.11(\mathrm{~m}, 12 \mathrm{H}), 7.05-6.99(\mathrm{~m}, 12 \mathrm{H}), 4.60(\mathrm{q}, J=6.8 \mathrm{~Hz}, 12 \mathrm{H}), 4.50(\mathrm{~d}$, $J=6.9 \mathrm{~Hz}, 6 \mathrm{H}), 2.49$ (d, $J=8.5 \mathrm{~Hz}, 12 \mathrm{H}), 1.88(\mathrm{br}, 3 \mathrm{H}), 1.52-1.50(\mathrm{~m}, 12 \mathrm{H}), 1.33(\mathrm{t}, J=7.0 \mathrm{~Hz}$, 9H), $1.24-1.21(\mathrm{~m}, 36 \mathrm{H}), 0.79-0.50(\mathrm{~m}, 51 \mathrm{H}), 0.30-0.24(\mathrm{~m}, 9 \mathrm{H}) .{ }^{13} \mathrm{C} \mathrm{NMR}\left(125 \mathrm{MHz}, \mathrm{CDCl}_{3}\right)$ $\delta: 178.75,162.71,161.30,160.45,157.71,149.83,148.76,142.14,142.03,141.50,141.27,140.69$, $131.21,128.77,127.88,127.78,125.22$, 120.30, 108.56, 105.25, 104.26, 62.82, 51.05, 44.10, 43.35, 37.82 , 35.74, 31.88, 31.54, 31.49, 29.35, 29.33, 29.17, 29.08, 22.77, 14.28, 14.06, 14.04, 14.02, 12.78, 12.57, 9.98, 9.90, 9.65, 9.58. HR-MS (MALDI-TOF) $\mathrm{m} / z$ calcd. for $\left(\mathrm{C}_{162} \mathrm{H}_{195} \mathrm{~N}_{9} \mathrm{O}_{6} \mathrm{~S}_{6}\right)$ : 2555.35881. Found: 2555.35923. Anal. Calcd. for $\mathrm{C}_{162} \mathrm{H}_{195} \mathrm{~N}_{9} \mathrm{O}_{6} \mathrm{~S} 6$ : C, 76.10\%; H, 7.69\%; N, 4.93\%. Found: C, 76.03\%; H, 7.74\%; N, 4.92\%. Solubility in chlorobenzene: $\sim 58 \mathrm{mg} / \mathrm{mL}$.

2,2',2"-((2Z,2'Z,2'Z)-((6,13,20-Tris(2-ethylhexyl)-4,4,11,11,18,18-hexakis(4-hexylphenyl)4,6,11,13,18,20-hexahydrothieno[2",3": $\left.3^{\prime}, 4^{\prime}\right]$ cyclopenta $\left[1^{\prime}, 2^{\prime}: 5,6\right]$ indolo[3,2 -

a]thieno[2", $\left.3^{\prime \prime}: 3^{\prime}, 4^{\prime}\right]$ cyclopenta[ $\left.1^{\prime}, 2^{\prime}: 5,6\right]$ indolo[3,2-c]thieno[2',3':3,4]cyclopenta[1,2h]carbazole-2,9,16-triyl)tris(methanylylidene))tris(5,6-difluoro-3-oxo-2,3-dihydro-1H-indene2,1-diylidene))trimalononitrile (TF2) 


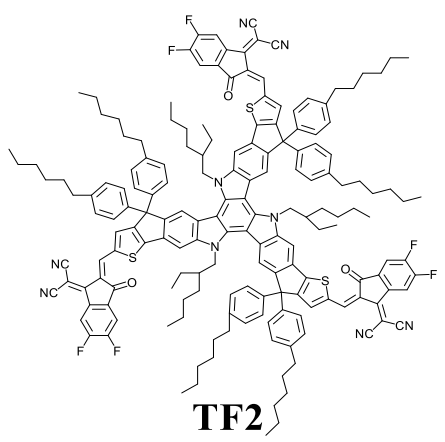

Compound 16 (200 mg, 0.10 mmol), 2-(5,6-difluoro-3-oxo-2,3-dihydro-1H-inden-1ylidene)malononitrile (138 $\mathrm{mg}, 0.60 \mathrm{mmol})$ and $\beta$-alanine $(2 \mathrm{mg})$ were dissolved in the mixture of dichloroethane and ethanol $(20 \mathrm{~mL}, v / v, 3 / 1)$ in a two-neck round-bottom flask. Then the resultant mixture was stirred at reflux overnight. Then the solvent was removed under reduced pressure and the crude product was purified by column chromatography (DCM) on silica gel to afford a brown powder as the desired product TF2 (148 mg, 56\% yield). ${ }^{1} \mathrm{H}$ NMR (500 $\left.\mathrm{MHz}, \mathrm{CDCl}_{3}\right) \delta: 8.83$ (s, $3 \mathrm{H}), 8.45\left(\mathrm{dd}, J_{1}=9.8 \mathrm{~Hz}, J_{2}=3.2 \mathrm{~Hz}, 3 \mathrm{H}\right), 8.09(\mathrm{~s}, 3 \mathrm{H}), 7.80(\mathrm{~s}, 3 \mathrm{H}), 7.73(\mathrm{~s}, 3 \mathrm{H}), 7.60(\mathrm{t}, J=$ $7.5 \mathrm{~Hz}, 3 \mathrm{H}), 7.18-7.14(\mathrm{~m}, 12 \mathrm{H}), 7.07-7.03(\mathrm{~m}, 12 \mathrm{H}), 4.65-4.63(\mathrm{~m}, 6 \mathrm{H}), 2.53-2.48(\mathrm{~m}, 12 \mathrm{H})$, 1.91 (br, 3H), 1.55-1.48 (m, 12H), 1.26-1.16 (m, 36H), 0.80-0.49 (m, 51H), 0.39-0.29 (m, 9H). ${ }^{13} \mathrm{C}$ NMR (125 MHz, $\left.\mathrm{CDCl}_{3}\right) \delta: 186.36,163.00,158.55,158.32,155.62,155.50,153.54,153.41$, $149.24,142.25,141.62$, 141.30, 140.73, 140.46, 138.80, 136.78, 136.69, 134.64, 131.05, 128.85, $127.90,127.80,125.53,120.30,120.25,115.23,115.06,114.69,114.62,112.70,112.55,105.29$, $104.70,68.88,62.85,51.12,35.75,31.89,31.88,31.53,31.49,29.86,29.35,29.32$, 28.07, 27.98, 27.86, 27.77, 22.77, 22.76, 14.26, 14.05, 14.03, 10.08, 9.96, 9.73, 9.64. H-F decoupled ${ }^{19} \mathrm{~F}$ NMR (376 MHz, $\left.\mathrm{CDCl}_{3}\right): \delta:-123.10(\mathrm{~d}, J=19.3 \mathrm{~Hz}, 3 \mathrm{~F}), 124.28(\mathrm{~d}, J=19.3 \mathrm{~Hz}, 3 \mathrm{~F}) . \mathrm{H}-\mathrm{F}$ coupled ${ }^{19} \mathrm{~F}$ NMR (376 MHz, CDCl3): $\delta:-123.06 \sim-123.13(\mathrm{~m}, 3 \mathrm{~F}),-124.24 \sim-124.29$ (m, 3F). HR-MS (MALDI-TOF) $m / z$ calcd. for $\left(\mathrm{C}_{174} \mathrm{H}_{171} \mathrm{~F}_{6} \mathrm{~N}_{9} \mathrm{O}_{3} \mathrm{~S}_{3}\right)$ : 2645.26048. Found: 2645.26064. Anal. Calcd. for $\mathrm{C}_{174} \mathrm{H}_{171} \mathrm{~F}_{6} \mathrm{~N}_{9} \mathrm{O}_{3} \mathrm{~S}_{3}: \mathrm{C}, 78.97 \% ; \mathrm{H}, 6.51 \% ; \mathrm{N}, 4.76 \%$. Found: C, 78.92\%; H, 6.55\%; N, 4.72\%. Solubility in chlorobenzene: $\sim 47 \mathrm{mg} / \mathrm{mL}$. 
4-((7-(6,13,20-Tris(2-ethylhexyl)-4,4,11,11,18,18-hexakis(4-hexylphenyl)-4,6,11,13,18,20-

hexahydrothieno $\left[3^{\prime \prime}, 2^{\prime \prime}: 4^{\prime}, 5^{\prime}\right]$ cyclopenta $\left[1^{\prime}, 2^{\prime}: 5,6\right]$ indolo $[3,2$ -

a]thieno[ $\left.3^{\prime \prime}, 2^{\prime \prime}: 4^{\prime}, 5^{\prime}\right]$ cyclopenta[ $\left.1^{\prime}, 2^{\prime}: 5,6\right]$ indolo $[3,2-c]$ thieno $\left[3^{\prime}, 2^{\prime}: 4,5\right]$ cyclopenta[1,2-

h]carbazol-2-yl)benzo[c][1,2,5]thiadiazol-4-yl)ethynyl)benzoic acid (DT1)

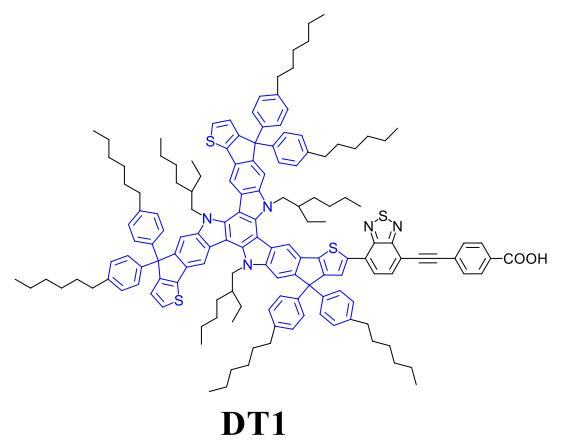

In a two-neck round bottom flask was dissolved T1 $(600 \mathrm{mg}, 0.31 \mathrm{mmol})$ in anhydrous THF (10 $\mathrm{mL})$ and cooled to $-78{ }^{\circ} \mathrm{C}$ using a dry ice/acetone bath. $n$-BuLi $(0.18 \mathrm{~mL}, 0.45 \mathrm{mmol}, 2.5 \mathrm{M}$ in hexane) was added to the reaction mixture dropwise. Then the resulting solution was stirred at the same temperature for $1 \mathrm{~h}$. Then chlorotrimethylstannane $(90 \mathrm{mg}, 0.45 \mathrm{mmol}$ ) was added and the resulting mixture was warmed to room temperature slowly and stirred for another $2 \mathrm{~h}$. Water (50 $\mathrm{mL}$ ) was added to terminate the reaction and the mixture was extracted three times with chloroform $(50 \mathrm{~mL} \times 3)$ before the organic phase was washed with water and dried over anhydrous sodium sulfate. After solvent removal under reduced pressure, the crude product was used in the next reaction directly. To a dried Schlenk tube were added the above organotin, butyl 4-((7bromobenzo[c][1,2,5]thiadiazol-4-yl)ethynyl)benzoate (141 mg, $0.45 \mathrm{mmol}), \mathrm{Pd}_{2}\left(\mathrm{dba}_{3}\right)_{3}(9 \mathrm{mg}$, $0.01 \mathrm{mmol}), \mathrm{P}(o \text {-tol })_{3}(20 \mathrm{mg}, 0.01 \mathrm{mmol})$ and toluene $(30 \mathrm{~mL})$ in a nitrogen-filled glovebox. The reaction mixture was refluxed under argon for $3 \mathrm{~h}$. Water $(100 \mathrm{~mL})$ was added and the mixture was extracted three times with chloroform $(50 \mathrm{~mL} \times 3)$. The organic phase was washed with water and then dried over anhydrous sodium sulfate. After solvent removal under reduced pressure, the residue was purified by column chromatography (toluene/petroleum ether $40-60^{\circ} \mathrm{C}, v / v, 1 / 1$ ) on silica gel to afford a black solid as the desired dye precursor. In a three-neck round bottom flask, dye precursor and $\mathrm{KOH}(224 \mathrm{mg}, 4.00 \mathrm{mmol})$ were dissolved in $\mathrm{THF} / \mathrm{H}_{2} \mathrm{O}(18 \mathrm{~mL}, v / v, 3 / 1)$. The mixture was stirred at reflux for $8 \mathrm{~h}$. Water $(50 \mathrm{~mL})$ and chloroform $(80 \mathrm{~mL})$ was added. The organic phase was washed with $0.2 \mathrm{M}$ phosphoric acid aqueous solution $(50 \mathrm{~mL})$ and water $(50$ $\mathrm{mL}$ ) in turn, and then dried over anhydrous sodium sulfate. After removing the solvent under 
reduced pressure, the crude product was purified by column chromatography (chloroform/methanol, $v / v, 15 / 1$ ) on silica gel to afford a black solid as the final product DT1 (464 $\mathrm{mg}, 68 \%$ yield). ${ }^{1} \mathrm{H} \mathrm{NMR}\left(500 \mathrm{MHz}, \mathrm{CDCl}_{3}\right) \delta: 9.01(\mathrm{~d}, J=8.4 \mathrm{~Hz}, 1 \mathrm{H}), 8.54-8.41(\mathrm{~m}, 5 \mathrm{H}), 8.32$ (s, 1H), $8.26(\mathrm{~s}, 1 \mathrm{H}), 8.19-8.08(\mathrm{~m}, 5 \mathrm{H}), 7.96-7.84(\mathrm{~m}, 4 \mathrm{H}), 7.52-7.46(\mathrm{~m}, 12 \mathrm{H}), 7.09-7.07$ (m, $12 \mathrm{H}), 4.65-4.62(\mathrm{~m}, 6 \mathrm{H}), 2.52-2.46(\mathrm{~m}, 12 \mathrm{H}), 1.90(\mathrm{br}, 3 \mathrm{H}), 1.55-1.47$ (m, 12H), 1.22-1.14 (m, $36 \mathrm{H}), 0.81-0.69(\mathrm{~m}, 51 \mathrm{H}), 0.61-0.48(\mathrm{~m}, 9 \mathrm{H})$. The peaks are very broad because of the strong aggregation of dipolar dyes in $\mathrm{CDCl}_{3}$. HR-MS (MALDI-TOF) $m / z$ calcd. for $\left(\mathrm{C}_{170} \mathrm{H}_{151} \mathrm{~N}_{5} \mathrm{O}_{2} \mathrm{~S}_{4}\right)$ : 2203.2349. Found: 2203.2358. Anal. Calcd. for $\mathrm{C}_{170} \mathrm{H}_{151} \mathrm{~N}_{5} \mathrm{O}_{2} \mathrm{~S}_{4}: \mathrm{C}, 81.73 \% ; \mathrm{H}, 7.82 \%$; N, 3.18\%. Found: C, 81.68\%; H, 7.86\%; N, 3.21\%.

4-((7-(6,13,20-Tris(2-ethylhexyl)-4,4,11,11,18,18-hexakis(4-hexylphenyl)-4,6,11,13,18,20hexahydrothieno[2", 3": 3',4']cyclopenta[1',2':5,6]indolo[3,2 -

a]thieno[2", $\left.3^{\prime \prime}: 3^{\prime}, 4^{\prime}\right]$ cyclopenta[ $\left[1^{\prime}, 2^{\prime}: 5,6\right]$ indolo[3,2-c]thieno[2',3':3,4]cyclopenta[1,2h]carbazol-2-yl)benzo[c][1,2,5]thiadiazol-4-yl)ethynyl)benzoic acid (DT2)

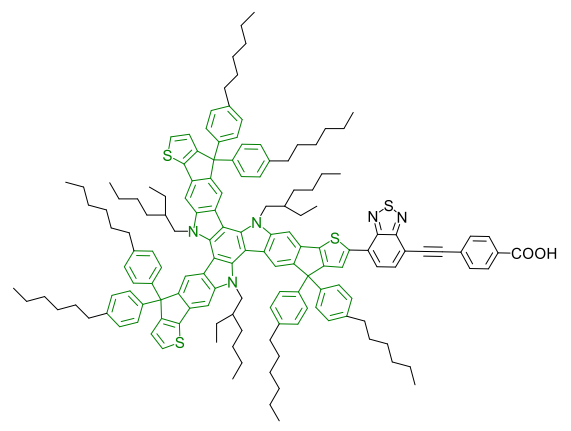

\section{DT2}

In a two-neck round bottom flask was dissolved T2 $(500 \mathrm{mg}, 0.26 \mathrm{mmol})$ in anhydrous THF (10 $\mathrm{mL})$ and cooled to $-78{ }^{\circ} \mathrm{C}$ using a dry ice/acetone bath. $n$ - BuLi $(0.15 \mathrm{~mL}, 0.38 \mathrm{mmol}, 2.5 \mathrm{M}$ in hexane) was added to the reaction mixture dropwise. Then the resulting solution was stirred at the same temperature for $1 \mathrm{~h}$. Then chlorotrimethylstannane $(75 \mathrm{mg}, 0.38 \mathrm{mmol}$ ) was added and the resulting mixture was warmed to room temperature slowly and stirred for another $2 \mathrm{~h}$. Water (50 $\mathrm{mL}$ ) was added to terminate the reaction and the mixture was extracted three times with chloroform $(50 \mathrm{~mL} \times 3)$ before the organic phase was washed with water and dried over anhydrous sodium sulfate. After solvent removal under reduced pressure, the crude product was used in the next reaction directly. To a dried Schlenk tube were added the above organotin, butyl 4-((7bromobenzo[c][1,2,5]thiadiazol-4-yl)ethynyl)benzoate (119 mg, $0.38 \mathrm{mmol}), \mathrm{Pd}_{2}(\mathrm{dba})_{3}(9 \mathrm{mg}$, 
$0.01 \mathrm{mmol}), \mathrm{P}(o-\text { tol })_{3}(20 \mathrm{mg}, 0.01 \mathrm{mmol})$ and toluene $(30 \mathrm{~mL})$ in a nitrogen-filled glovebox. The reaction mixture was refluxed under argon for $3 \mathrm{~h}$. Water $(100 \mathrm{~mL})$ was added and the mixture was extracted three times with chloroform $(50 \mathrm{~mL} \times 3)$. The organic phase was washed with water and then dried over anhydrous sodium sulfate. After solvent removal under reduced pressure, the residue was purified by column chromatography (toluene/petroleum ether $40-60{ }^{\circ} \mathrm{C}, v / v, 1 / 1$ ) on silica gel to afford a black solid as the desired dye precursor. In a three-neck round bottom flask, dye precursor and $\mathrm{KOH}(224 \mathrm{mg}, 4.00 \mathrm{mmol})$ were dissolved in $\mathrm{THF} / \mathrm{H}_{2} \mathrm{O}(18 \mathrm{~mL}, v / v, 3 / 1)$. The mixture was stirred at reflux for $8 \mathrm{~h}$. Water $(100 \mathrm{~mL})$ and chloroform $(50 \mathrm{~mL})$ was added. The organic phase was washed with $0.2 \mathrm{M}$ phosphoric acid aqueous solution $(50 \mathrm{~mL})$ and water $(50$ $\mathrm{mL}$ ) in turn, and then dried over anhydrous sodium sulfate. After removing the solvent under reduced pressure, the crude product was purified by column chromatography (chloroform/methanol, $v / v, 15 / 1$ ) on silica gel to afford a black solid as the final product DT2 (372 mg, 65\% yield). ${ }^{1} \mathrm{H}$ NMR (500 MHz, $\left.\mathrm{CDCl}_{3}\right) \delta: 9.03(\mathrm{~d}, J=8.4 \mathrm{~Hz}, 1 \mathrm{H}), 8.59-8.46$ (m, 5H), 8.48 (s, 1H), $8.36(\mathrm{~s}, 1 \mathrm{H}), 8.22-8.11(\mathrm{~m}, 5 \mathrm{H}), 7.97-7.85(\mathrm{~m}, 4 \mathrm{H}), 7.64-7.52(\mathrm{~m}, 12 \mathrm{H}), 7.06-7.01(\mathrm{~m}$, $12 \mathrm{H}), 4.68-4.63(\mathrm{~m}, 6 \mathrm{H}), 2.49-2.43(\mathrm{~m}, 12 \mathrm{H}), 1.89$ (br, 3H), 1.56-1.44 (m, 12H), 1.19-1.10 (m, $36 \mathrm{H}), 0.82-0.67(\mathrm{~m}, 51 \mathrm{H}), 0.60-0.43(\mathrm{~m}, 9 \mathrm{H})$. The peaks are very broad because of the strong aggregation of dipolar dyes in $\mathrm{CDCl}_{3}$. HR-MS (MALDI-TOF) $\mathrm{m} / \mathrm{z}$ calcd. for $\left(\mathrm{C}_{170} \mathrm{H}_{151} \mathrm{~N}_{5} \mathrm{O}_{2} \mathrm{~S}_{4}\right)$ : 2203.2349. Found: 2203.2361. Anal. Calcd. for $\mathrm{C}_{170} \mathrm{H}_{151} \mathrm{~N}_{5} \mathrm{O}_{2} \mathrm{~S}_{4}: \mathrm{C}, 81.73 \% ; \mathrm{H}, 7.82 \%$;, $3.18 \%$. Found: C, $81.71 \% ; \mathrm{H}, 7.85 \%$; N, 3.19\%.

1.2 Instrumentation. Bruker DRX 400 NMR and Bruker DRX 500 NMR spectrometers were employed to record the ${ }^{1} \mathrm{H}$ NMR and ${ }^{13} \mathrm{C}$ NMR spectra in a solution of $\mathrm{CDCl}_{3}$. High resolution mass spectra were obtained by AutoFlex II mass spectrometer using MALDI-TOF method. UVvis-NIR electronic absorption spectra in the solvent and soild state were recorded by a Varian Cary 5000. LifeSpec-II fluorescence spectrometer was used to get the PL spectra and an EPL485 laser diode to provide the excitation light. The electrochemical measurements were conducted on a CHI660C electrochemical workstation with $0.1 \mathrm{M} \mathrm{Bu} 4 \mathrm{NPF}_{6}$ as the supporting electrolyte. A threeelectrode electrolytic cell with a glassy carbon working electrode, a platinum gauze counter electrode, and a silver wire quasi-reference electrode was used in our nonaqueous electrochemical measurements and an $i R$ drop was compensated.

1.3 Theoretical Calculations. The quantum chemical calculations of donor cores T1, T2 and their 
corresponding electron acceptors and dye were conducted on the Gaussian 09 software package by using of the $6-31 \mathrm{G}(\mathrm{d}, \mathrm{p})$ basis set. ${ }^{[\mathrm{S} 7]}$ The geometries of ground-state molecules were optimized by selecting the widely used B3LYP exchange-correlation function and the simulation for solvent effects was performed by virtue of the conductor-like polarized continuum model (C-PCM). ${ }^{\text {[S8] }}$ The calculation of the vertical electron transition was implemented by using the TD-MPW1K hybrid functional. ${ }^{[\mathrm{S} 9]}$

1.4 Device Fabrication. Organic solar cells (OSCs), characteristic of a traditional structure of ITO/PEDOT:PSS/active layer/C60-bissalt/Ag, were fabricated. The ITO-coated glass substrates were cleaned using ultrasonic agitation in acetone, detergent, deionized water and isopropanol in turns, which was further treated by plasma for 3 min. The PEDOT:PSS (Baytron PVP 4083) layer on the ITO glass was fabricated by spin-coating at $4000 \mathrm{rpm}$ for $30 \mathrm{~s}$ and further annealed for 10 min at $150{ }^{\circ} \mathrm{C}$. In a glovebox filled with $\mathrm{N}_{2}$, the active layer solutions (the weight ratio of polymer donor/electron acceptor is $1: 1$ and the concentration of the active layer precursor is $20 \mathrm{mg} \mathrm{ml}^{-1}$ ) were dissolved in CB (chlorobenzene). Then the active layers on the substrates were obtained by spin coating the active layer precursor with $3000 \mathrm{rpm}$ speed and the light harvesting film of 100 $\mathrm{nm}$ thickness can be formed. Finally, the $2 \mathrm{mg} / \mathrm{ml} \mathrm{C60-bissalt} \mathrm{dissolved} \mathrm{in} \mathrm{methanol} \mathrm{was} \mathrm{spin-}$ coated on top of active layer and the counter electrode was covered by depositing $150 \mathrm{~nm} \mathrm{Ag}$ in vacuum chamber of $10^{-7}$ Torr. The device area is $\sim 4 \mathrm{~mm}^{2}$ and a metal mask with an aperture aligned with the device area was used during the performance tests. For the fabrication of dye sensitized solar cells (DSCs), a titania coated fluorine doped tin oxide (FTO) conducting glass (NSG, Solar, $4.0 \mathrm{~mm}$ ) was used as the negative electrode of DSCs which is in detail described in the literatures. ${ }^{[\mathrm{S} 10]}$ In general, a bilayer titania film was deposited on a sheet of conducting glass by using of screen-printing and sintering. The small $\mathrm{TiO}_{2}$ particles $(\sim 30 \mathrm{~nm})$ composed the 4.0$\mu \mathrm{m}$-thick translucent layer and the large particles $(250-300 \mathrm{~nm})$ composed the $4.5-\mu \mathrm{m}$-thick lightscattering layer. Dye-loading of DT1 was carried out by immersing a titania electrode into a dye bath solution for $12 \mathrm{~h}$. The solutions were composed of $150 \mu \mathrm{M}$ dye molecules in the mixture of ethanol and chloroform $(v / v, 1 / 9)$. A $25-\mu$ m-thick Surlyn ring was heated at $130{ }^{\circ} \mathrm{C}$ to adhere a dyed titania electrode and an Au/Cr coated FTO (NSG, TEC7, $2.2 \mathrm{~mm}$ ) electrode. The internal space of a partly sealed cell was infiltrated with a cobalt electrolyte. The Co-bpy electrolyte is composed of $0.25 \mathrm{M}$ tris(2,2'-bipyridiene)cobalt(II) di[bis(trifluoromethanesulfonyl)imide], 0.05 $\mathrm{M}$ tris(2,2'-bipyridiene)cobalt(III) tris[bis(trifluoromethanesulfonyl)imide], 0.5 M TBP, and 0.1 M 
LiTFSI in acetonitrile. The Co-phen electrolyte is composed of $0.25 \mathrm{M}$ tris(1,10phenanthroline)cobalt(II) $\quad \operatorname{di}[$ bis(trifluoromethanesulfonyl)imide], $\quad 0.05 \quad \mathrm{M} \quad \operatorname{tris}(1,10-$ phenanthroline)cobalt(III) tris[bis(trifluoromethanesulfonyl)imide], 0.5 M TBP, and 0.1 M LiTFSI in acetonitrile. An antireflection film (ASAHI, ARKTOP, $\lambda<380 \mathrm{~nm}$ ) was laminated onto the photoanode of DSCs.

1.5 Characterization of solar cells. The EQE spectra were recorded by a assembled setup including a stable light source which is powered by a Xenon lamp, light chopper, monochromator, and lockin amplifier. The $J$ - $V$ curves were measured by a Keithley 2400 source unit under a simulated solar irradiance (solar simulator from Newport Inc.) which can be calibrated to 1 sun $\left(100 \mathrm{~mW} \mathrm{~cm}^{\square 2}\right.$ ) illumination intensity by a certified silicon diode. A neutral filter was utilized to study the light intensity dependent device performance. A black metal mask was covered on a testing cell during EQE and $J-V$ measurements.

1.6 SCLC measurements. For SCLC measurement, the electron-only device with structure of ITO/ZnO/Active layer/C60-bissalt/Ag and the hole-only device with structure of ITO/PEDOT:PSS/Active layer/ $\mathrm{MoO}_{3} / \mathrm{Ag}$ were fabricated respectively. $2 \mathrm{mg}$ C60-bissalt was dissloved in $1 \mathrm{ml}$ methanol solution and spincoated on active layer at $2500 \mathrm{rpm}$ for $30 \mathrm{~s}$. The carrier mobilities were measured using the space-charge-limited-current (SCLC) model, which is described by:

$$
J=9 \varepsilon 0 \varepsilon r u V^{2} / 8 L^{3}
$$

where $\mathrm{J}$ is the current density, $\mathrm{L}$ is the film thickness of active layer, $\varepsilon_{0}$ is the permittivity of free space $\left(8.85 \times 10^{-12} \mathrm{~F} \mathrm{~m}^{-1}\right), \varepsilon_{\mathrm{r}}$ is the relative dielectric constant of the transport medium, $\mathrm{u}$ is the hole or electron mobility, $\mathrm{V}$ is the internal voltage in the device and $\mathrm{V}=\mathrm{Vappl-Vr}-\mathrm{Vbi}$, where Vappl is the applied voltage to the device, $\operatorname{Vr}$ is the voltage drop due to contact resistance and series resistance across the electrodes, and Vbi is the built-in voltage due to the relative work function difference of the two electrodes. The thickness of the BHJ blend for SCLC measurement was about $100 \mathrm{~nm}$. The electron mobility can be calculated from the slope of the $J^{1 / 2} \sim \mathrm{V}$ curves.

\subsection{Grazing Incidence Wide-Angle X-ray Scattering (GIWAXS) Characterization. GIWAXS} measurements were performed at beamline 7.3.3 $3^{[11]}$ at the Advanced Light Source. Samples were prepared on Si substrates using identical blend solutions as those used in devices. The $10 \mathrm{keV} \mathrm{X-}$ 
ray beam was incident at a grazing angle of $0.12^{\circ}-0.16^{\circ}$, selected to maximize the scattering intensity from the samples. The scattered x-rays were detected using a Dectris Pilatus 2M photon counting detector. The detailed methods for analysis of GIWAXS were described in corresponding literature. ${ }^{[12]}$

The information about the size of crystalline grains is contained in the width of the diffraction peaks, and at the simplest level the Scherrer equation can be employed to extract a grain size. The Scherrer equation relates the peak width to the crystal coherence length (CCL),

$$
\mathrm{CCL}=\frac{2 \pi K}{\Delta q}
$$

where $\mathrm{K}$ is a shape factor (typically $0.8-1$ ) and $\Delta q$ is the full width at half-maximum of a diffraction peak. It should be well understood that calling this a grain or crystallite size is rigorously correct when the only factor affecting the measured peak breadth is the finite size of the crystalline assemblies. 


\section{Additional Figures and Tables}

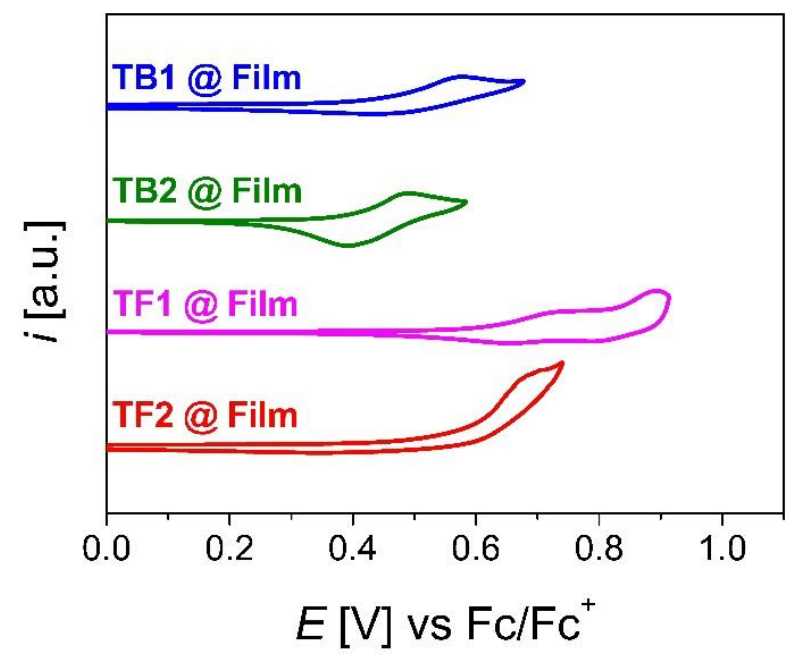

Figure S1. Cyclic voltammograms of TB1, TB2, TF1 and TF2 in the solid state with $0.1 \mathrm{M}$ $\mathrm{Bu}_{4} \mathrm{NPF}_{6}$ as the supporting electrolyte and the potential was further calibrated with respect to the standard redox couple, ferrocene/ferrocenium $\left(\mathrm{Fc} / \mathrm{Fc}^{+}\right)$. The corresponding iR compensation was also conducted and the scan rate was $5 \mathrm{mV} \mathrm{s}^{-1}$. The corresponding HOMO energy levels were viably estimated via the equation $E=-4.88-e E_{\text {onset }}$, where $E_{\text {onset }}$ is the onset oxidation potential of a ground-state molecule. 

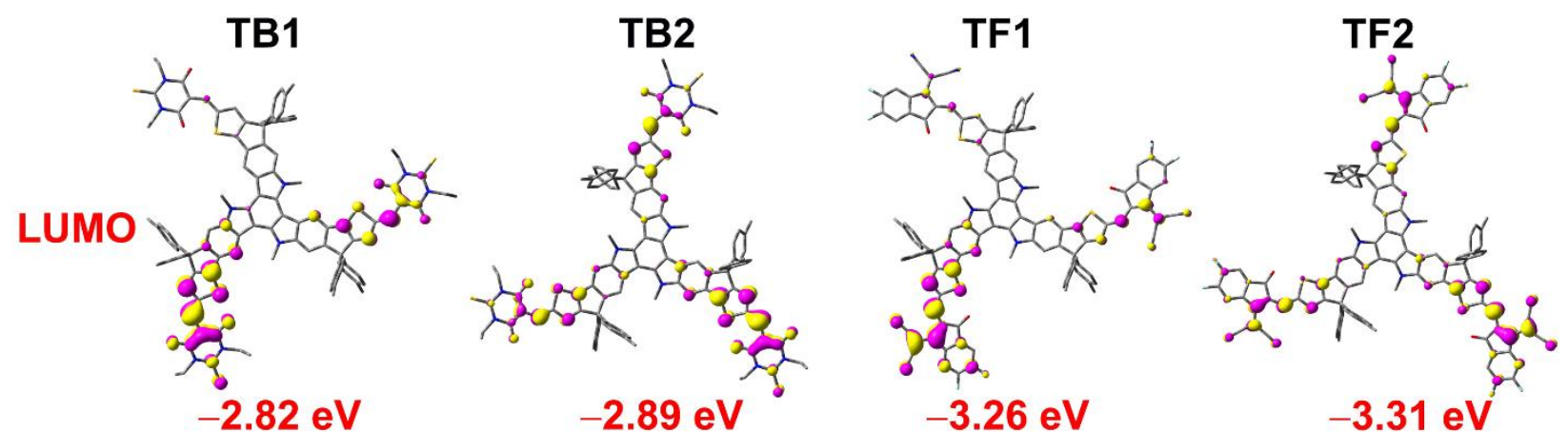

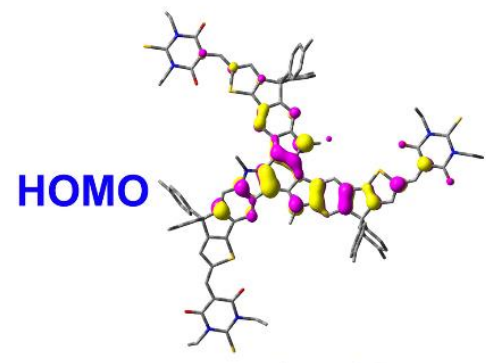

$-5.37 \mathrm{eV}$

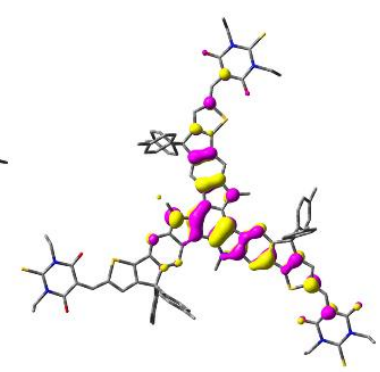

$-5.32 \mathrm{eV}$

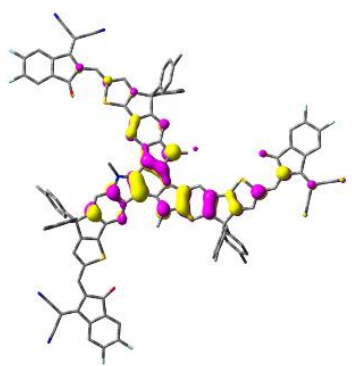

$-5.52 \mathrm{eV}$

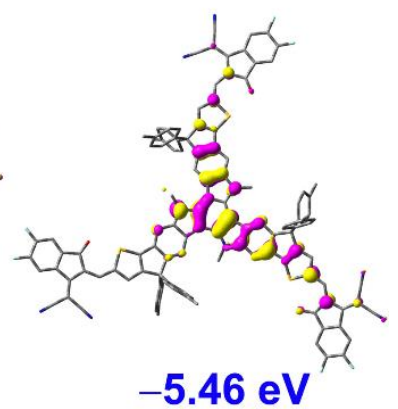

Figure S2. Geometry and contour plots of the NFA frontier molecular orbitals; LUMO and HOMO energy levels given for quantitative comparison. The isodensity surface values were fixed at 0.03 and the large aliphatic groups were substituted with methyl to accelerate computation. 

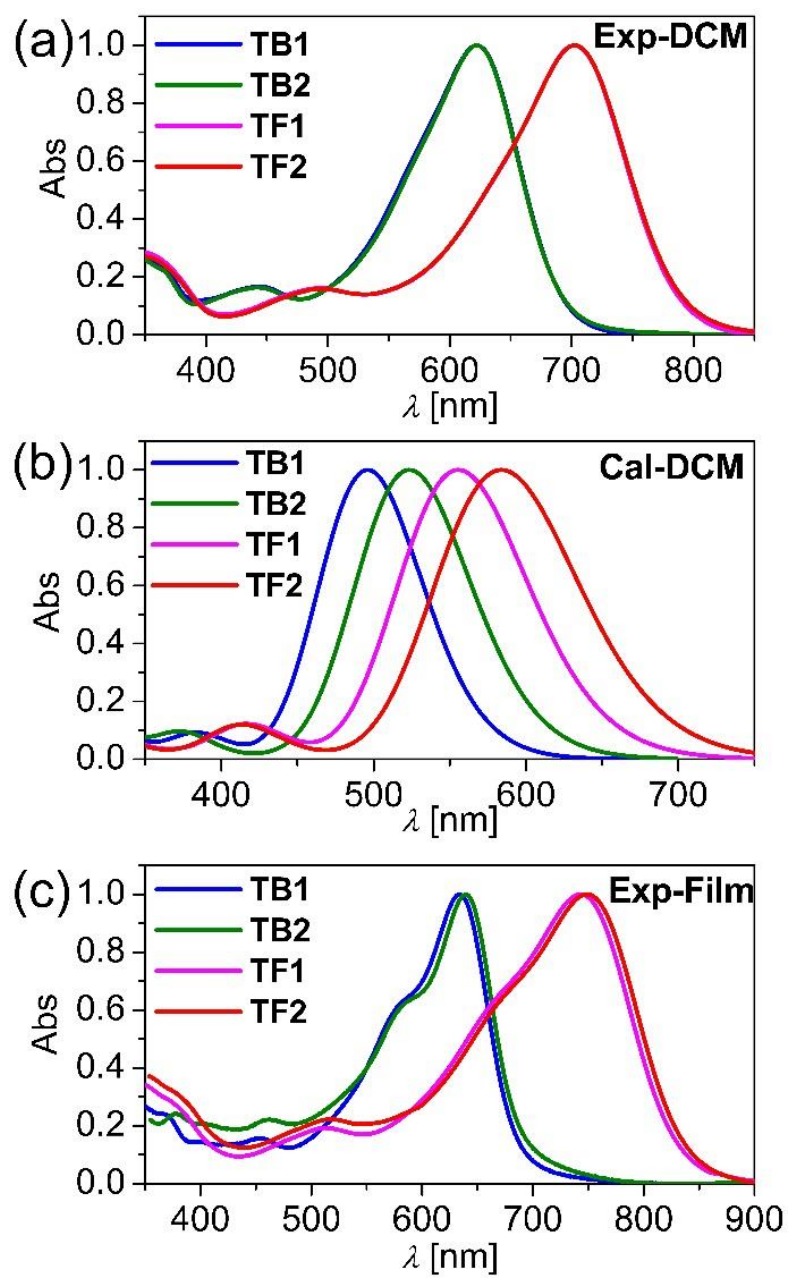

Figure S3. (a) Normalized electronic absorption spectra of four electron acceptors in DCM solutions. (b) Calculated electronic absorption spectra of four electron acceptors in DCM solutions by time-dependent DFT at the TD-MPW1K level. The peak half-width is fixed at $0.20 \mathrm{eV}$. (c) Normalized electronic absorption spectra of NFAs from solid state films. 


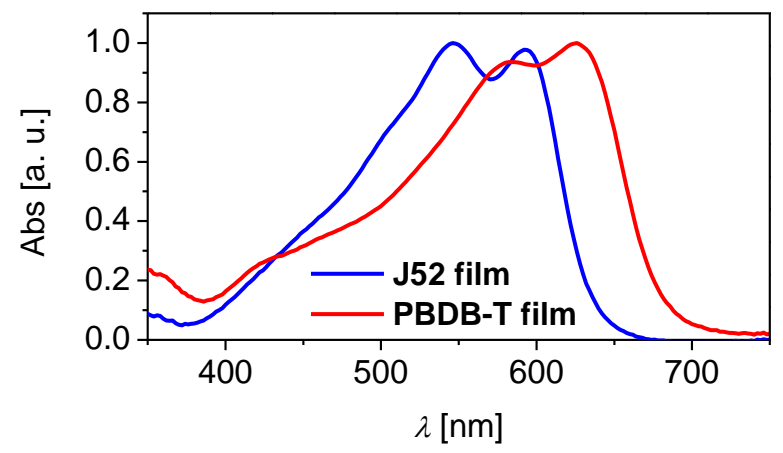

Figure S4. Absorption spectra of two polymer donors J52 and PBDB-T in solid state. 


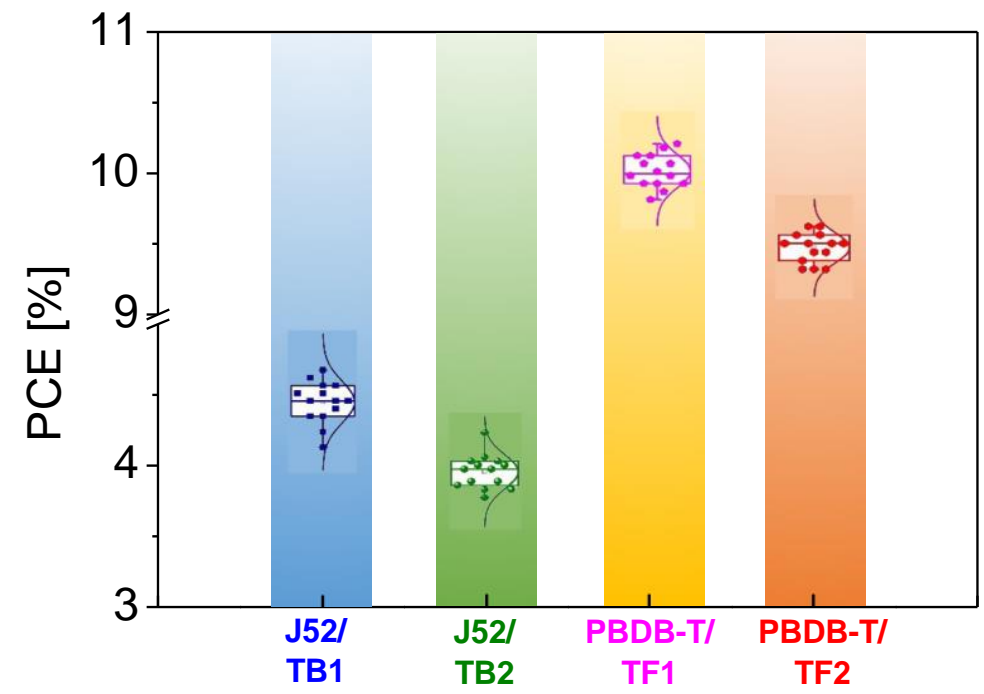

Figure S5. PCEs extracted from 15 different OSCs. 


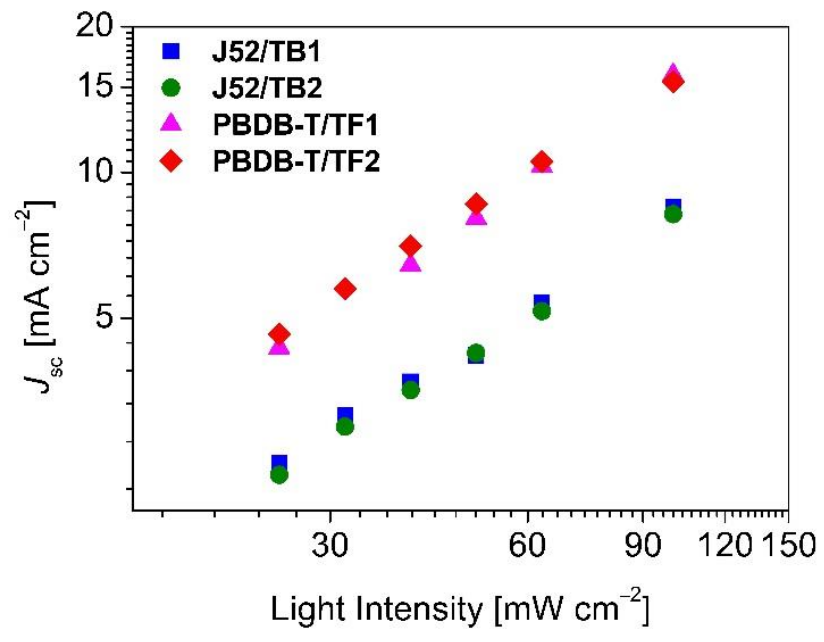

Figure S6. $J_{\mathrm{SC}}$ versus light intensity of the optimized devices. The formula $J_{\mathrm{SC}} \propto \mathrm{P}^{a}$ is generally utilized to evaluate the degree of bimolecular recombination. For T1 and T2 cores based OSCs, the $\alpha$ values are 0.97 for J52/TB1; 0.98 for J52/TB2; 0.98 for PBDB-T/TF1 and 0.98 for PBDBT/TF2, suggesting the negligible bimolecular charge recombination. 

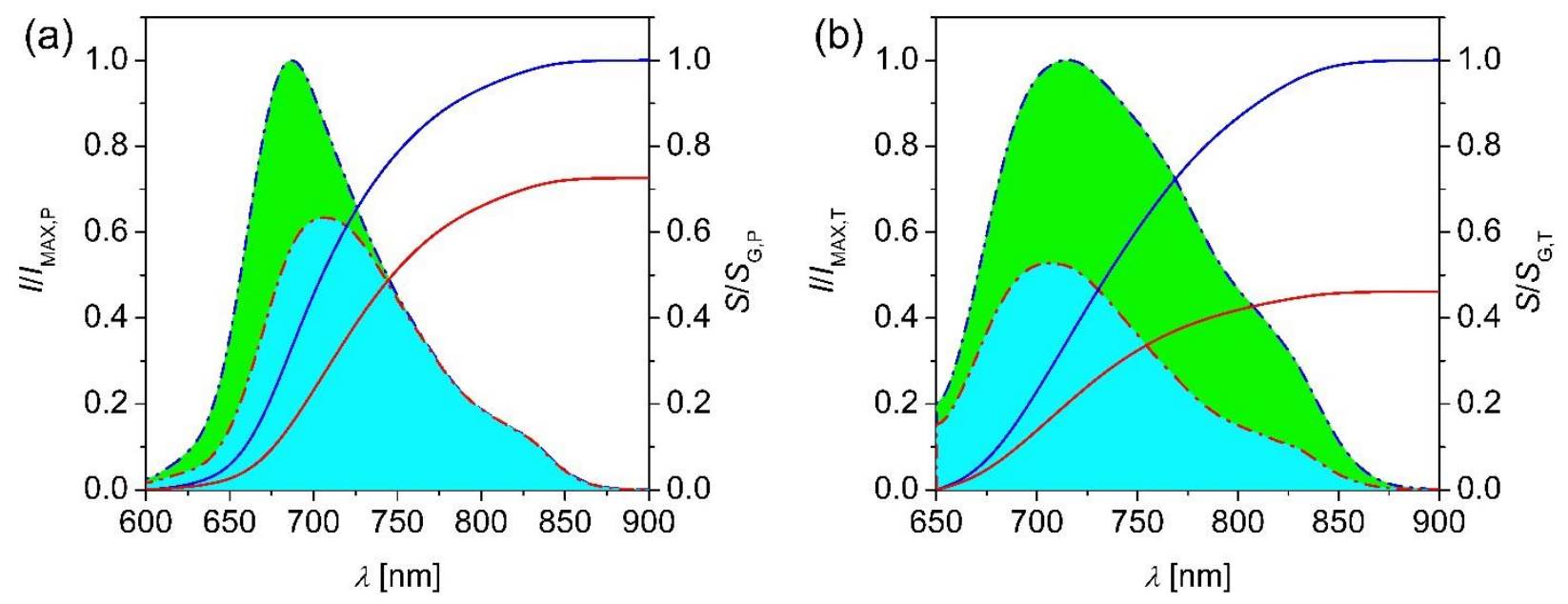

Figure S7. (a) Steady PL traces of pristine polymer donor PBDB-T film (blue dash lines) and its blended film with TB1 at the optimized conditions (red dash lines). The PL intensity (I) was

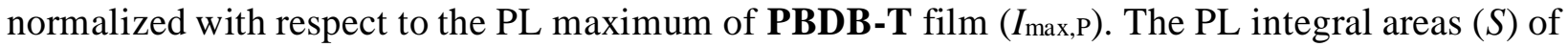
the PBDB-T (blue solid line) and PBDB-T/TB1 blend (red solid line) films were normalized with respect to the global PL integral area $\left(S_{\mathrm{G}, \mathrm{P}}\right)$ of PBDB-T film. Excitation wavelength: $550 \mathrm{~nm}$. PL quenching efficiency: 27\%. (b) Steady PL traces of TB1 film (blue dash lines) and its corresponding blended film with PBDB-T at the optimized conditions (red dash lines). The PL intensity $(I)$ was normalized with respect to the PL maximum of TB1 film $\left(I_{\max , T)}\right.$. The PL integral areas $(S)$ of the TB1 (blue solid line) and PBDB-T/TB1 blend (red solid line) films were normalized with respect to the global PL integral area $\left(S_{\mathrm{G}, \mathrm{T}}\right)$ of TB1 film. Excitation wavelength: $570 \mathrm{~nm}$. PL quenching efficiency: $54 \%$. 

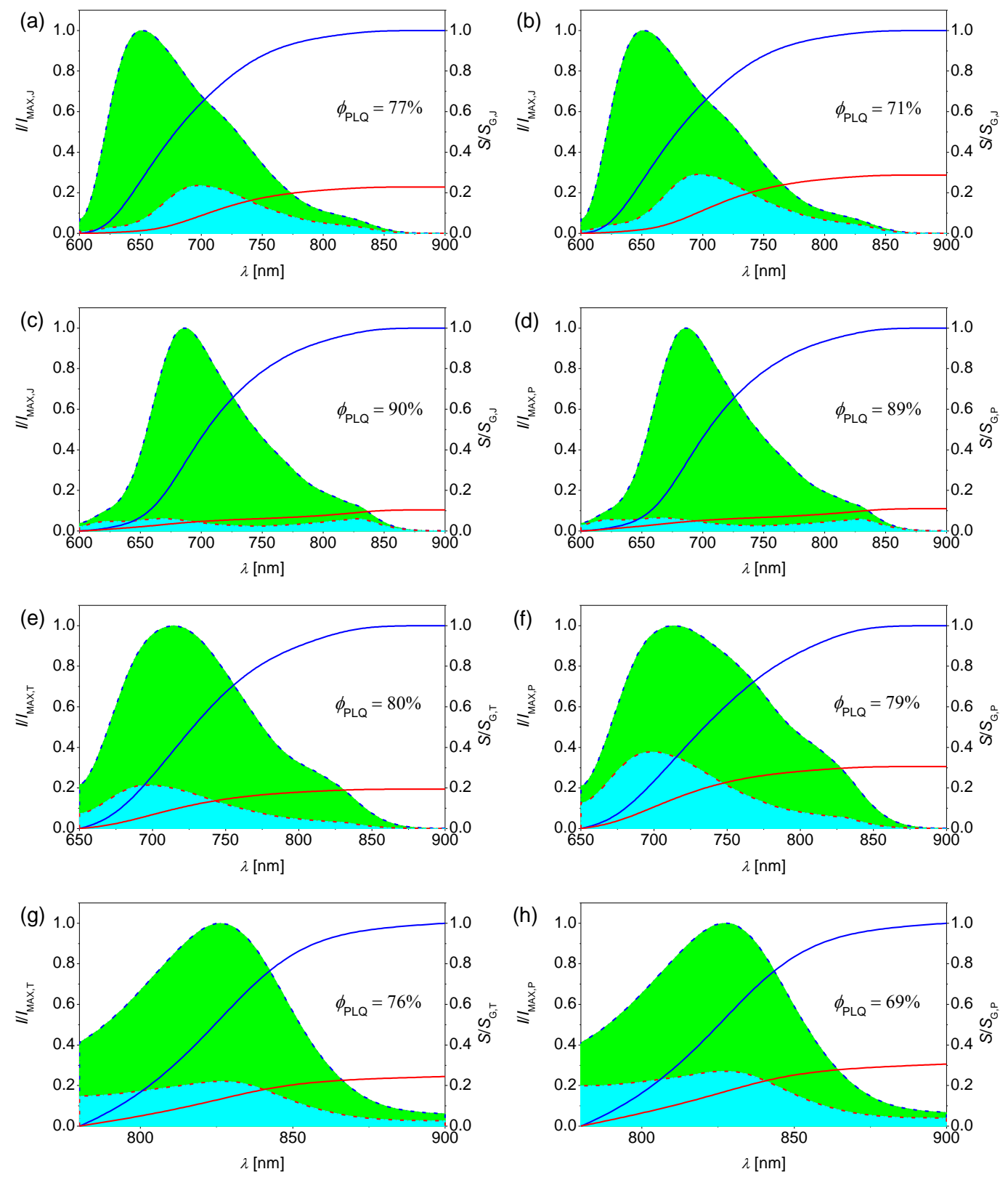

Figure S8. Steady PL and integral area traces of different samples. PL intensity $(I)$ and integral areas $(S)$ were normalized by the PL maximum $\left(I_{\max }\right)$ and global PL integral area $\left(S_{\mathrm{G}}\right)$ of pristine film respectively. PL quenching efficiencies were indicated in the figures. (a) PL of J52, blue dash; J52/TB1, red dash; Integral area of J52, blue solid; J52/TB1, red solid. Pump wavelength: 520 nm. (b) PL of J52, blue dash; J52/TB2, red dash; Integral area of J52, blue solid; J52/TB2, red solid. Pump wavelength: 520 nm. (c) PL of PBDB-T, blue dash; PBDB-T/TF1, red dash; Integral area of PBDB-T, blue solid; PBDB-T/TF1, red solid. Pump wavelength: $550 \mathrm{~nm}$. (d) PL of PBDB-T, blue dash; PBDB-T/TF2, red dash; Integral area of PBDB-T, blue solid; PBDB-T/TF2, 
red solid. Pump wavelength: $550 \mathrm{~nm}$. (e) PL of TB1, blue dash; J52/TB1, red dash; Integral area of TB1, blue solid; J52/TB1, red solid. Pump wavelength: $570 \mathrm{~nm}$. (f) PL of TB2, blue dash; J52/TB2, red dash; Integral area of TB2, blue solid; J52/TB2, red solid. Pump wavelength: 570 nm. (g) PL of TF1, blue dash; PBDB-T/TF1, red dash; Integral area of TF1, blue solid; PBDBT/TF1, red solid. Pump wavelength: $650 \mathrm{~nm}$. (h) PL of TF2, blue dash; PBDB-T/TF2, red dash; Integral area of TF2, blue solid; PBDB-T/TF2, red solid. Pump wavelength: $650 \mathrm{~nm}$. 


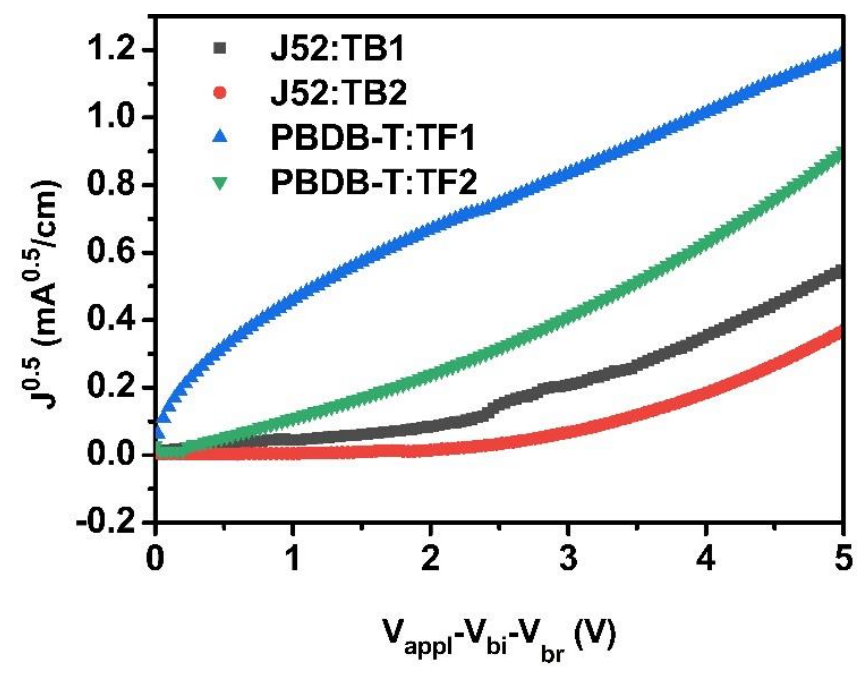

Figure S9. Electron mobilities of blend films. 


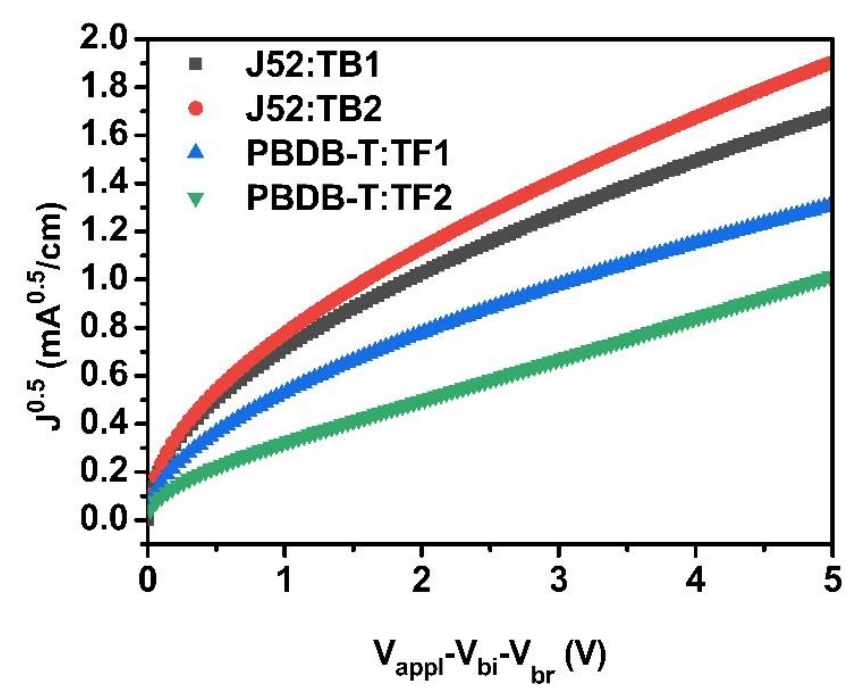

Figure S10. Hole mobilities of blend films. 


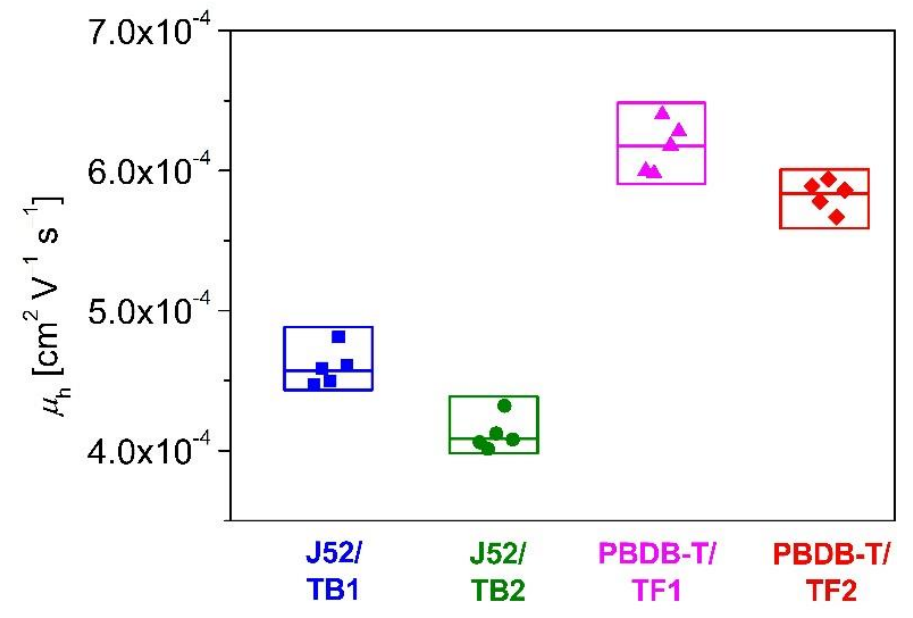

Figure S11. Electron hole mobilities $\left(\mu_{\mathrm{h}}\right)$ of blend films based on J52/TB1, J52/TB2, PBDBT/TF1 and PBDB-T/TF1. 


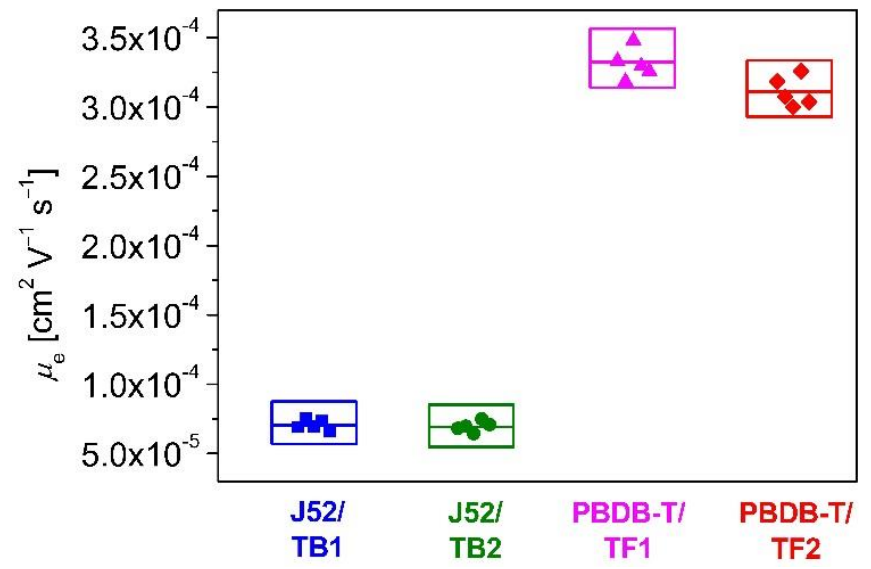

Figure S12. Electron mobilities $\left(\mu_{\mathrm{e}}\right)$ of blend films based on J52/TB1, J52/TB2, PBDB-T/TF1 and PBDB-T/TF2. 
Table S1. The Parameters of Hole and Electron Mobilities.

\begin{tabular}{|c|c|c|c|}
\hline Blend films & $\begin{array}{c}\mu_{\mathrm{h}}^{a} \\
{\left[10^{-4} \mathrm{~cm}^{2} \mathrm{~V}^{-1} \mathrm{~s}^{-1}\right]}\end{array}$ & $\begin{array}{c}\mu_{\mathrm{e}}^{b} \\
{\left[10^{-4} \mathrm{~cm}^{2} \mathrm{~V}^{-1} \mathrm{~s}^{-1}\right]}\end{array}$ & $\mu_{\mathrm{h}} / \mu_{\mathrm{e}}$ \\
\hline J52/TB1 & 4.6 & 0.7 & 6.6 \\
\hline J52/TB2 & 4.1 & 0.7 & 5.8 \\
\hline PBDB-T/TF1 & 6.2 & 3.3 & 1.8 \\
\hline PBDB-T/TF2 & 5.8 & 3.1 & 1.9 \\
\hline
\end{tabular}



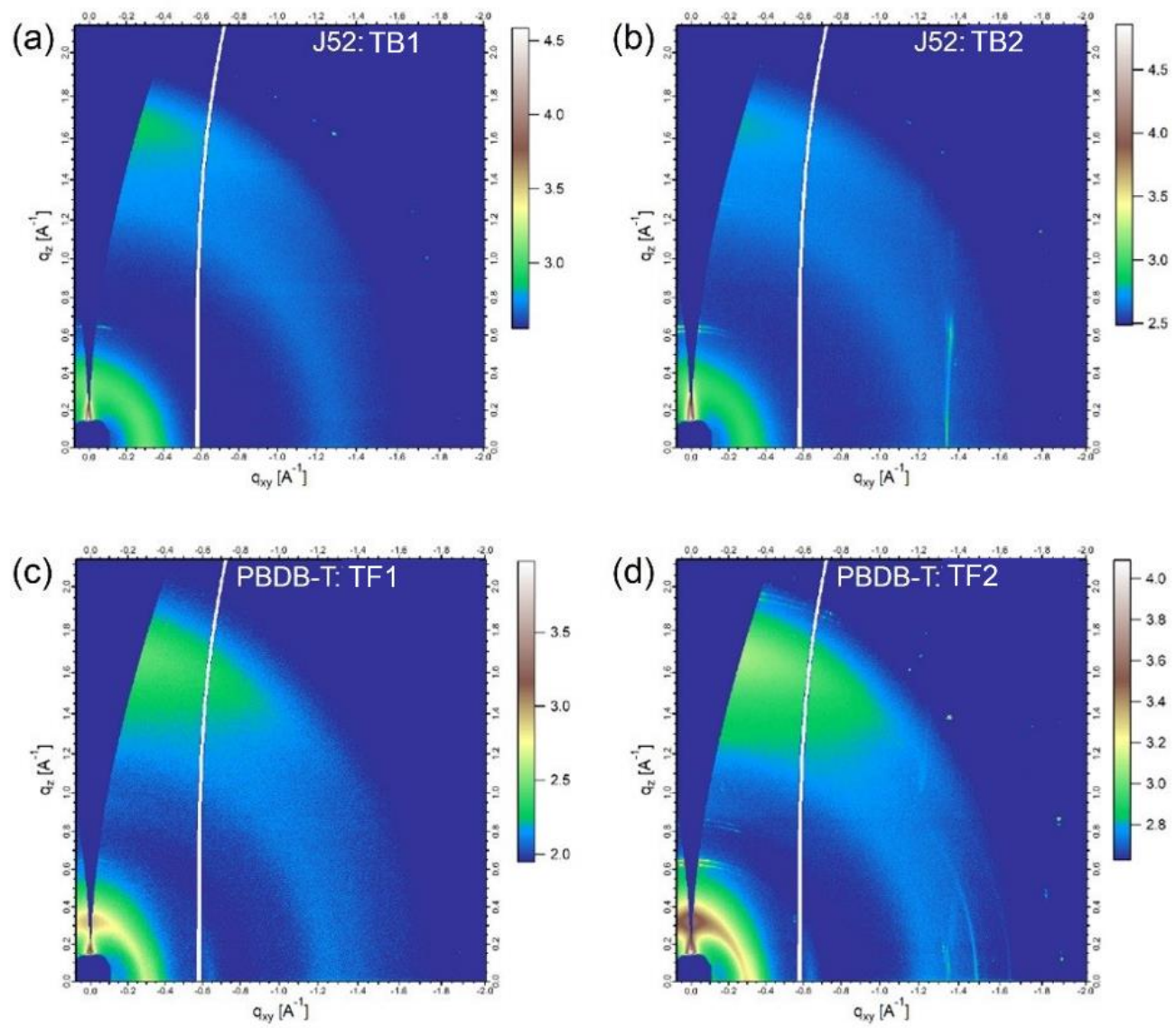

Figure S13. 2D GIWAXS patterns for blend films. 


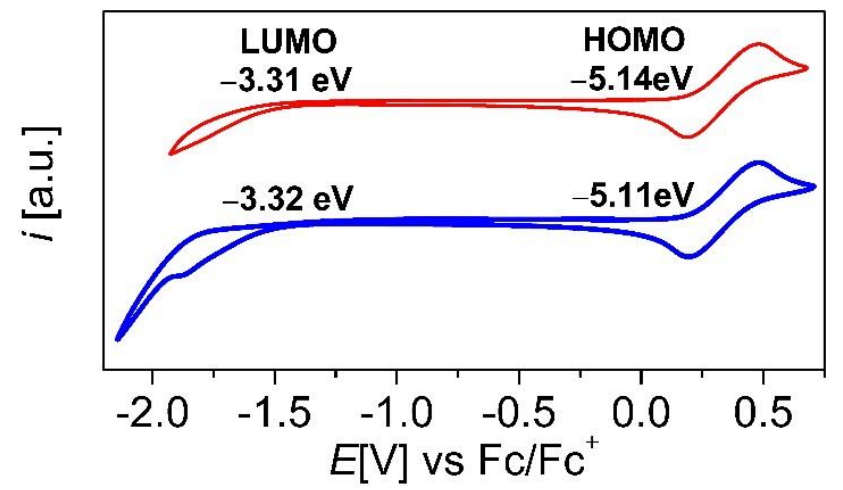

Figure S14. Cyclic voltammograms of DT1 (blue) and DT2 (red) dyes in DCM solutions with 0.1 $\mathrm{M} \mathrm{Bu}_{4} \mathrm{NPF}_{6}$ as the supporting electrolyte and the potential was further calibrated with respect to the standard redox couple, ferrocene/ferrocenium $\left(\mathrm{Fc} / \mathrm{Fc}^{+}\right)$. The corresponding iR compensation was also conducted and the scan rate was $5 \mathrm{mV} \mathrm{s}^{-1}$. The corresponding HOMO and LUMO energy levels were viably estimated via the equation $E=-4.88-e E_{\text {onset }}$, where $E_{\text {onset }}$ is the onset oxidation potential of a ground-state molecule. 


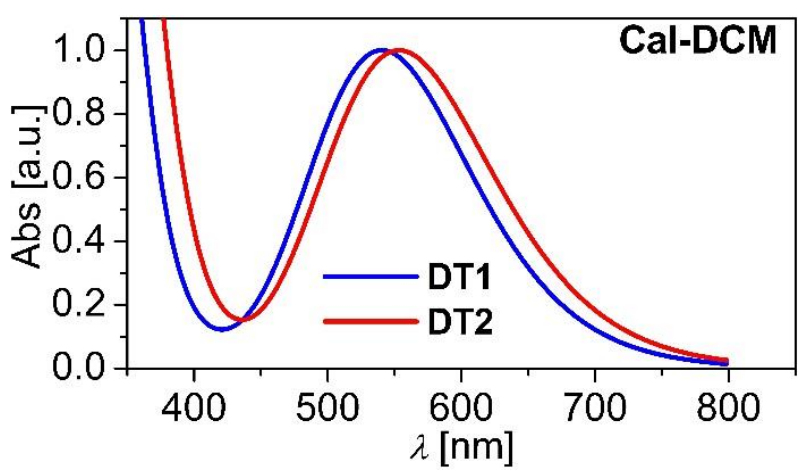

Figure S15. Calculated electronic absorption spectra of two dyes in DCM solutions by timedependent DFT at the TD-MPW1K level. The peak half-width is fixed at $0.20 \mathrm{eV}$. 


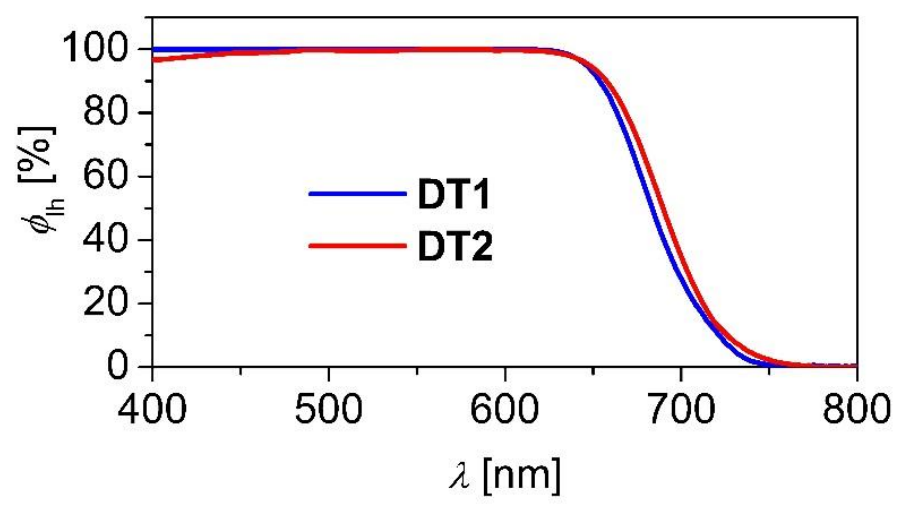

Figure S16. Plots of light-harvesting yield $\left(\phi_{\mathrm{LH}}\right)$ vs $(\lambda)$ for $8.0-\mu \mathrm{m}$-thick, dye-grafted mesoporous titania films immersed in a Co-bpy electrolyte. 
Table S2. Dual-Path Charge-Transfer Kinetics for the Reactions between Oxidized Dyes and Free Electrons.

\begin{tabular}{|c|c|c|c|}
\hline Dye & $t_{1 / 2-\text { rec }^{a}}$ & $t_{1 / 2}$-reg ${ }^{b}$ & $\phi_{\mathrm{hi}}^{c}$ \\
\hline DT1 & $965 \mu \mathrm{s}$ & $26 \mu \mathrm{s}$ & $97 \%$ \\
\hline DT2 & $880 \mu \mathrm{s}$ & $42 \mu \mathrm{s}$ & $93 \%$ \\
\hline
\end{tabular}


3. Spectral Charts of NMR

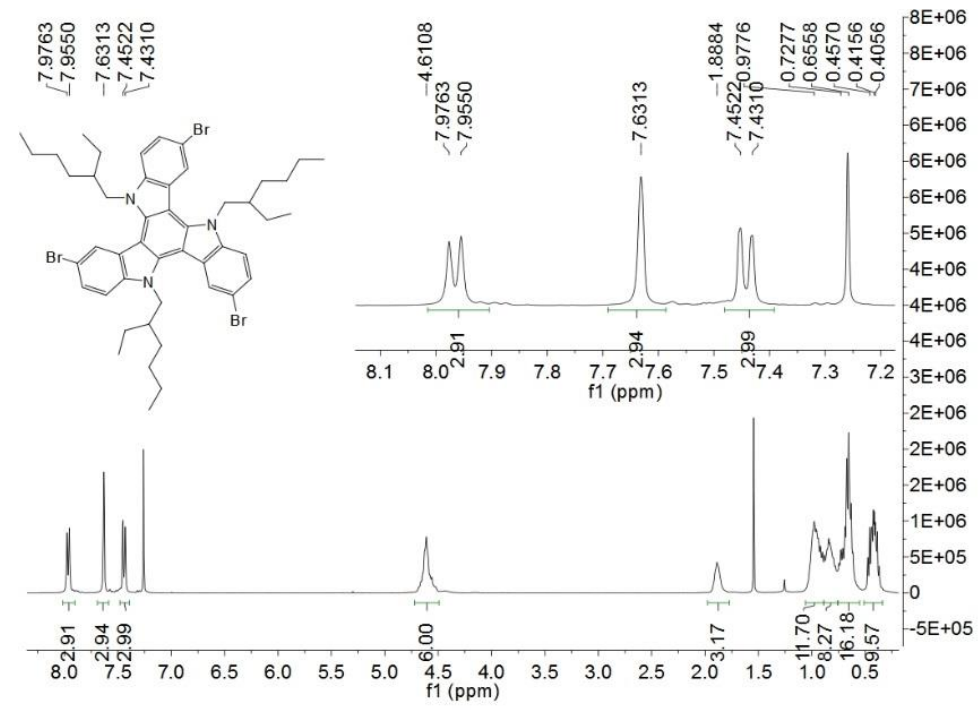

Figure S17. The ${ }^{1} \mathrm{H}$ NMR spectrum $(400 \mathrm{MHz})$ of 3 in $\mathrm{CDCl}_{3}$. 


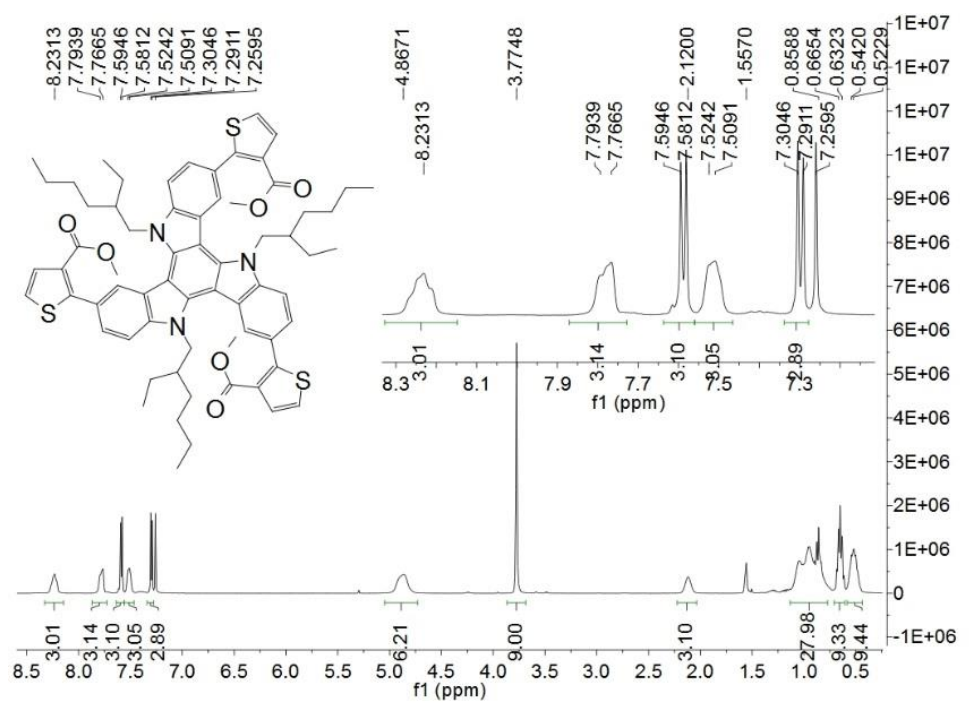

Figure S18. The ${ }^{1} \mathrm{H}$ NMR spectrum $(400 \mathrm{MHz})$ of 5 in $\mathrm{CDCl}_{3}$. 


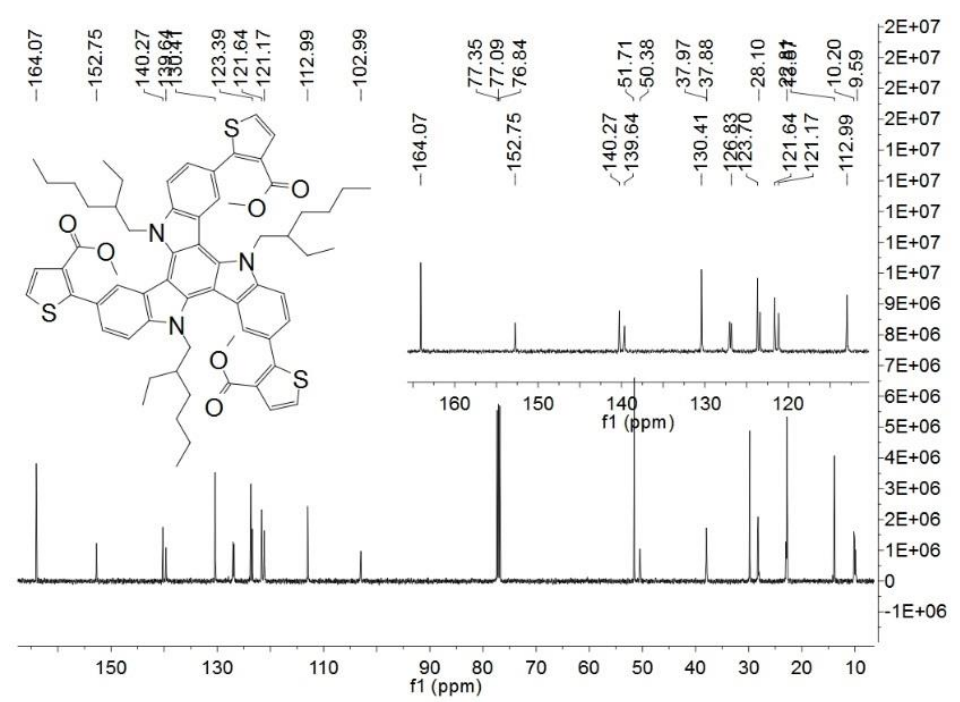

Figure S19. The ${ }^{13} \mathrm{C}$ NMR spectrum $(125 \mathrm{MHz})$ of 5 in $\mathrm{CDCl}_{3}$. 


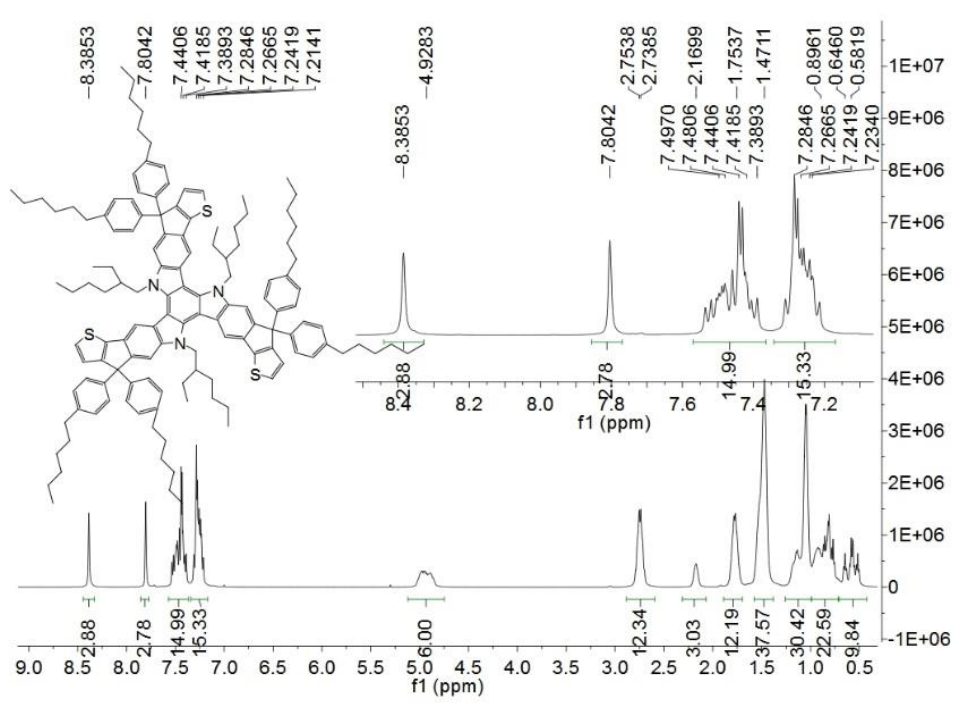

Figure S20. The ${ }^{1} \mathrm{H}$ NMR spectrum $(500 \mathrm{MHz})$ of $\mathbf{T} 1$ in $\mathrm{CDCl}_{3}$. 


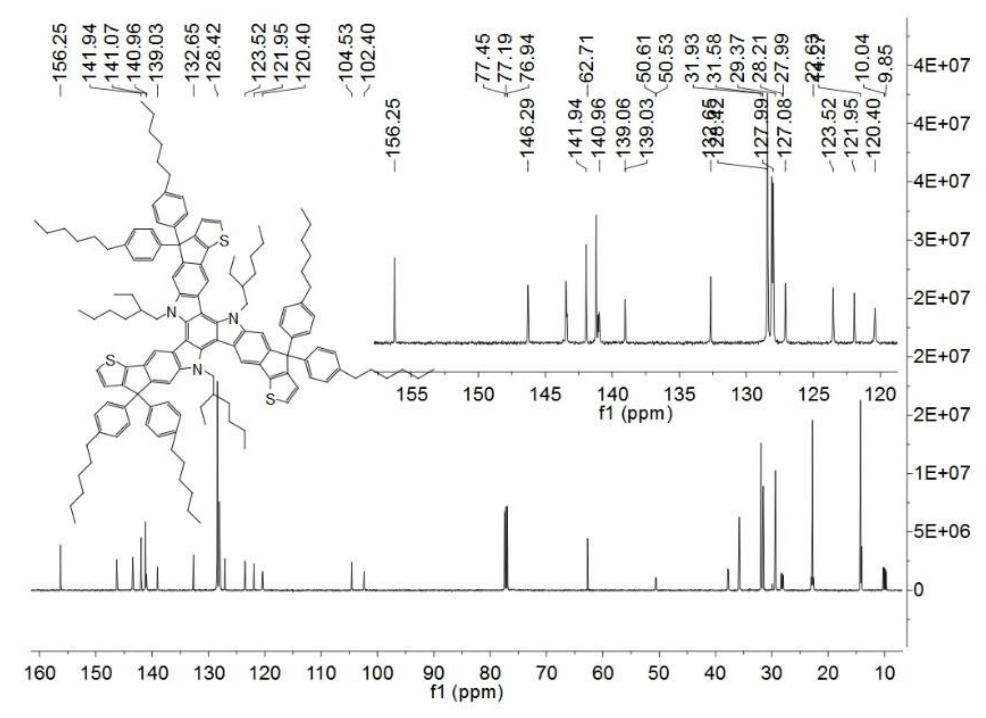

Figure S21. The ${ }^{13} \mathrm{C}$ NMR spectrum $(125 \mathrm{MHz})$ of $\mathbf{T} 1$ in $\mathrm{CDCl}_{3}$. 


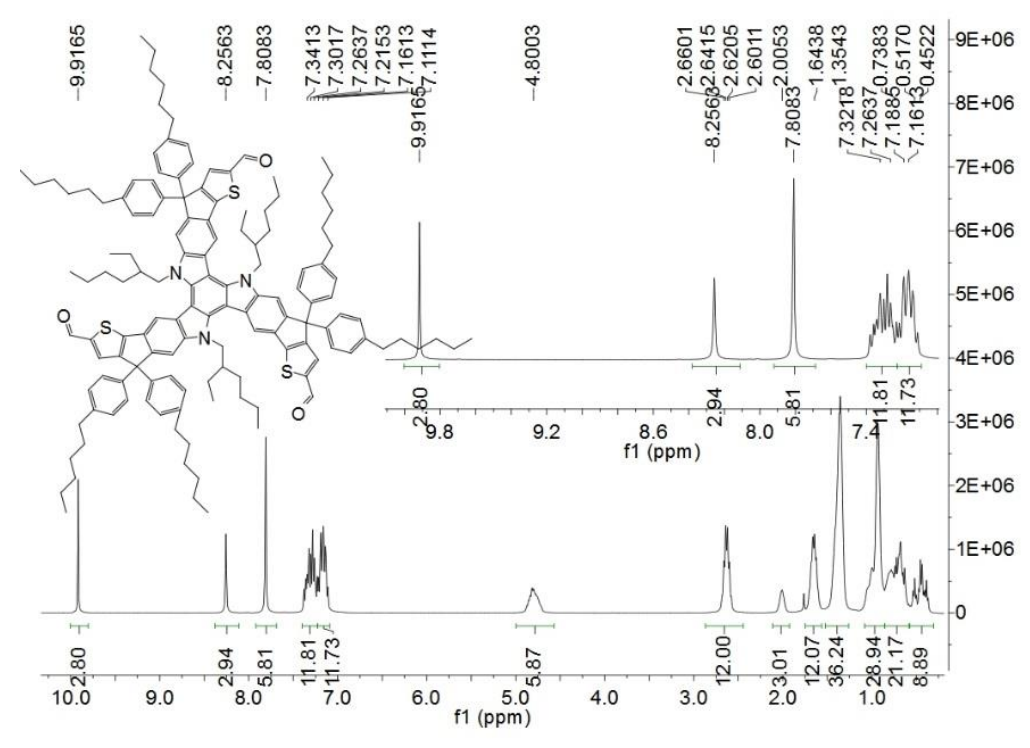

Figure S22. The ${ }^{1} \mathrm{H}$ NMR spectrum $(400 \mathrm{MHz})$ of 15 in $\mathrm{CDCl}_{3}$. 


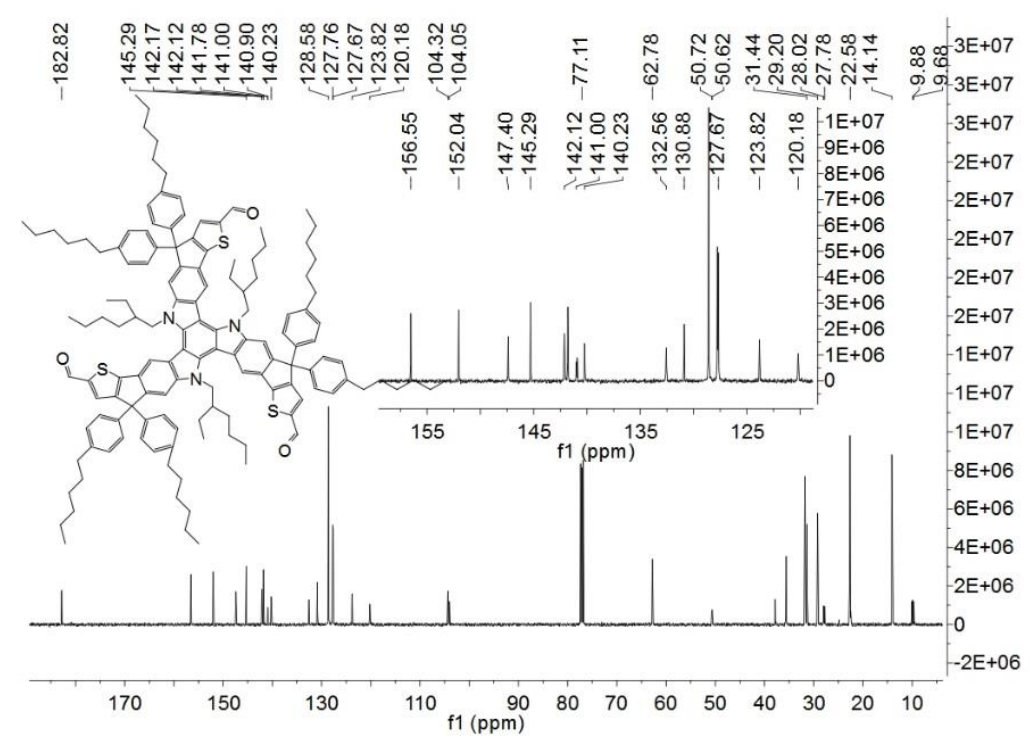

Figure S23. The ${ }^{13} \mathrm{C}$ NMR spectrum $(125 \mathrm{MHz})$ of 15 in $\mathrm{CDCl}_{3}$. 


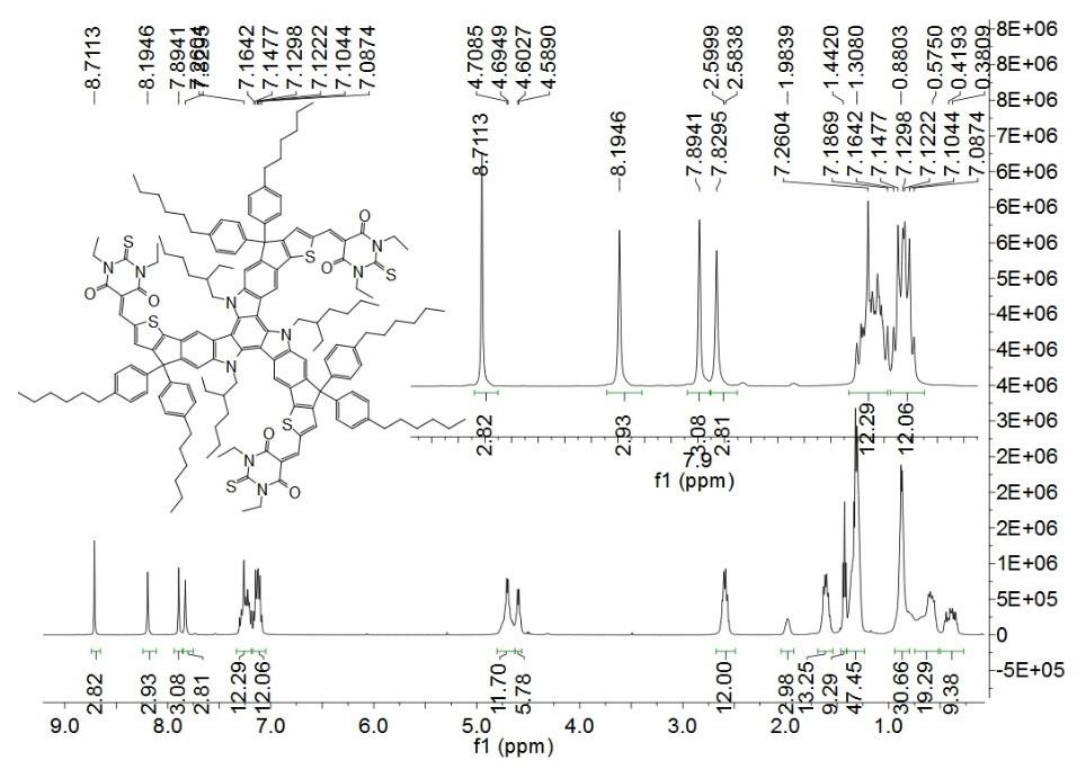

Figure S24. The ${ }^{1} \mathrm{H}$ NMR spectrum $(500 \mathrm{MHz})$ of TB1 in $\mathrm{CDCl}_{3}$. 


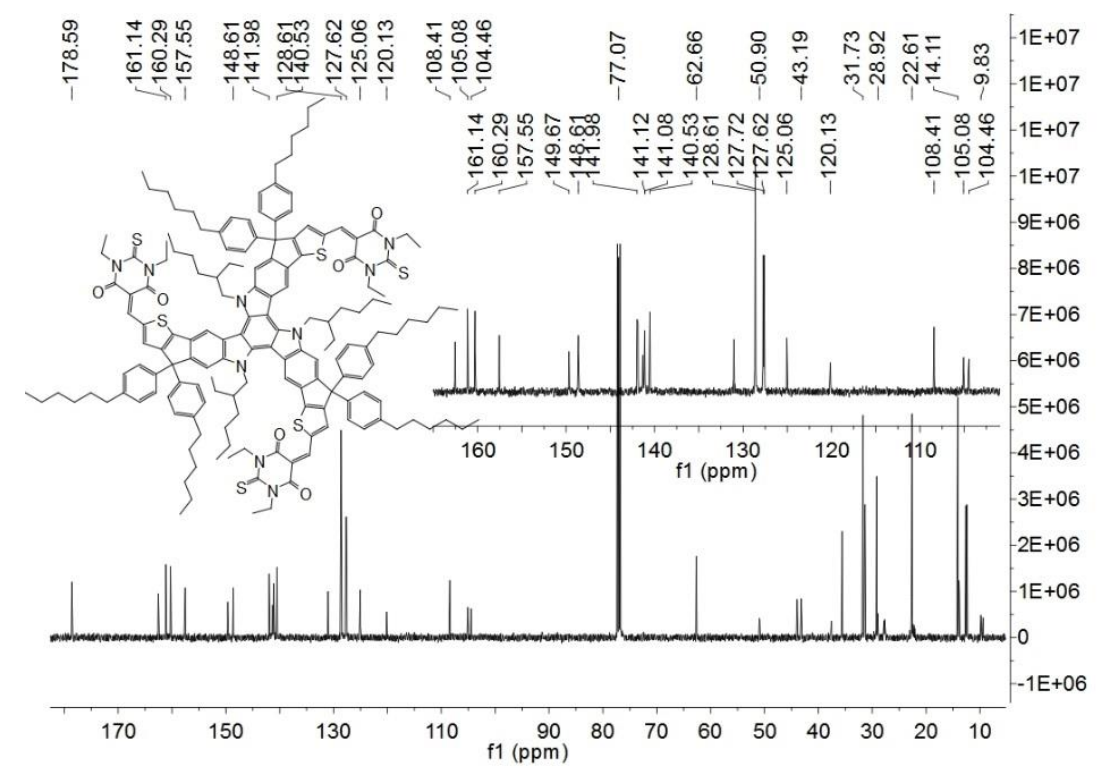

Figure S25. The ${ }^{13} \mathrm{C}$ NMR spectrum $(125 \mathrm{MHz})$ of $\mathbf{T B 1}$ in $\mathrm{CDCl}_{3}$. 


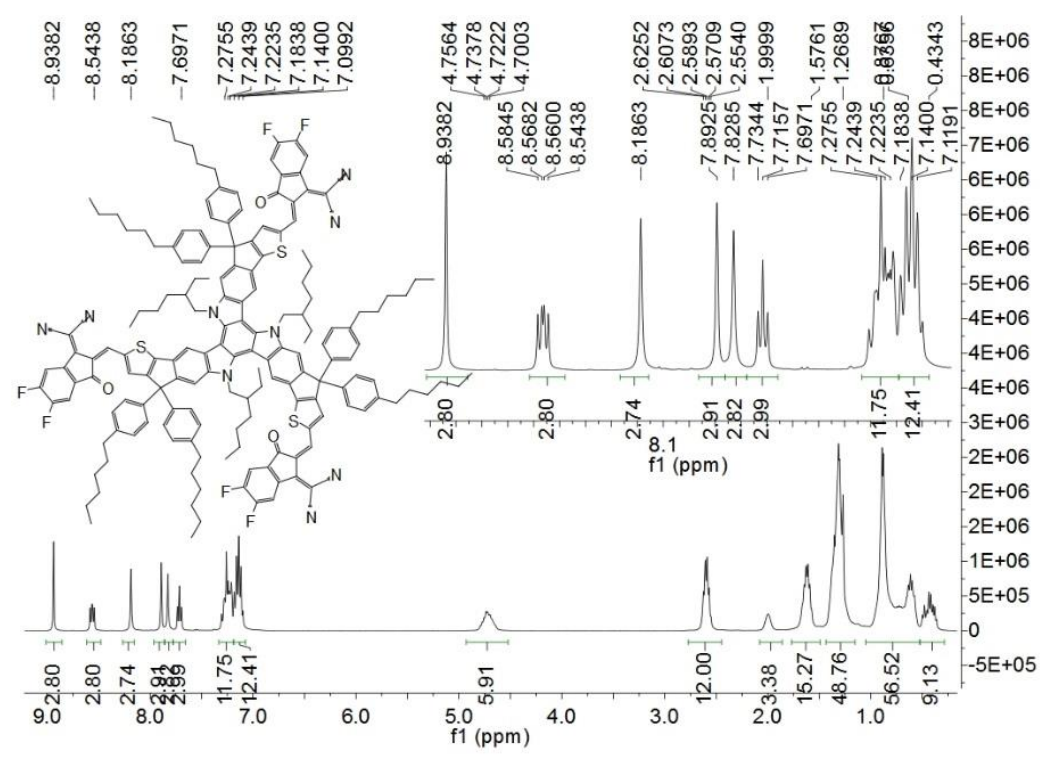

Figure S26. The ${ }^{1} \mathrm{H}$ NMR spectrum $(400 \mathrm{MHz})$ of TF1 in $\mathrm{CDCl}_{3}$. 


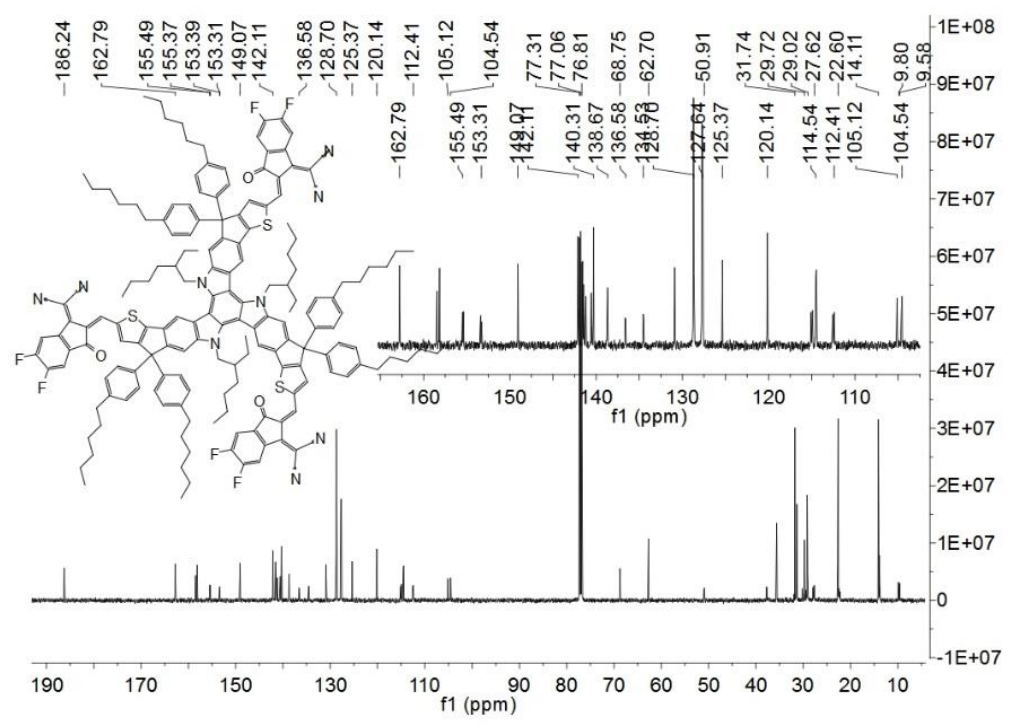

Figure S27. The ${ }^{13} \mathrm{C}$ NMR spectrum $(125 \mathrm{MHz})$ of TF1 in $\mathrm{CDCl}_{3}$. 


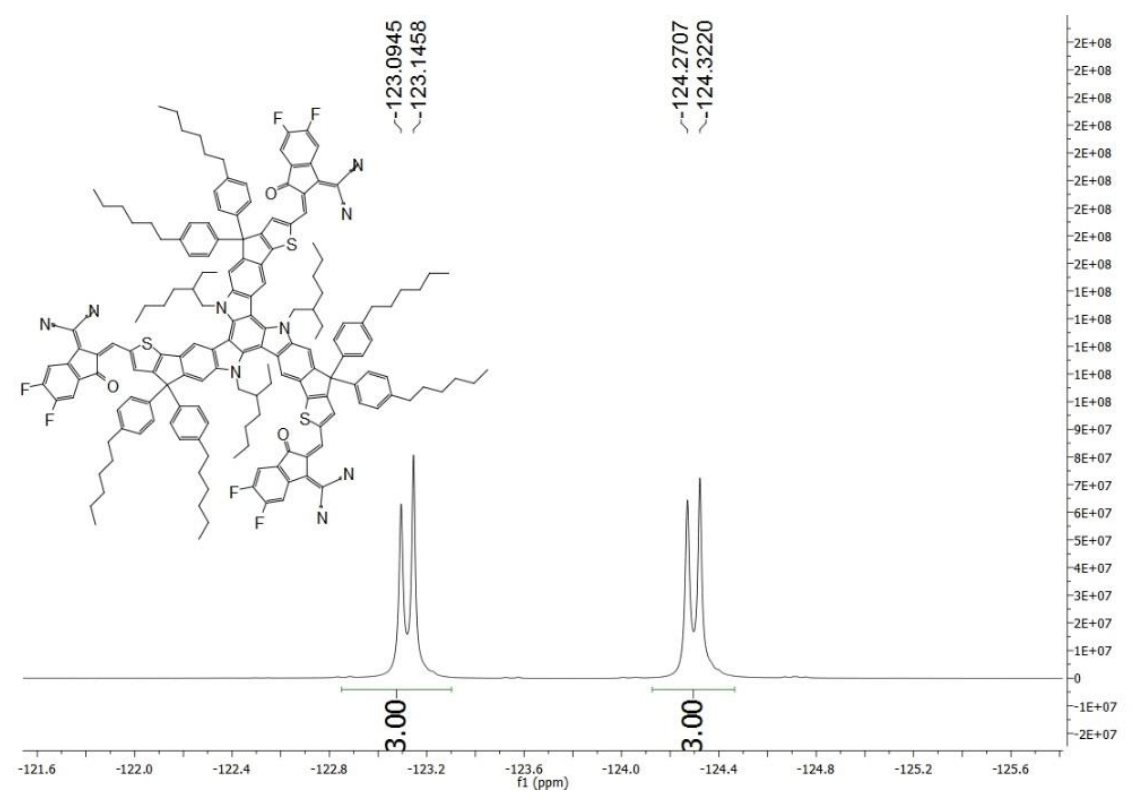

Figure S28. The 19F $\{1 \mathrm{H}\}$ NMR spectrum $(376 \mathrm{MHz})$ of TF1 in $\mathrm{CDCl}_{3}$. 


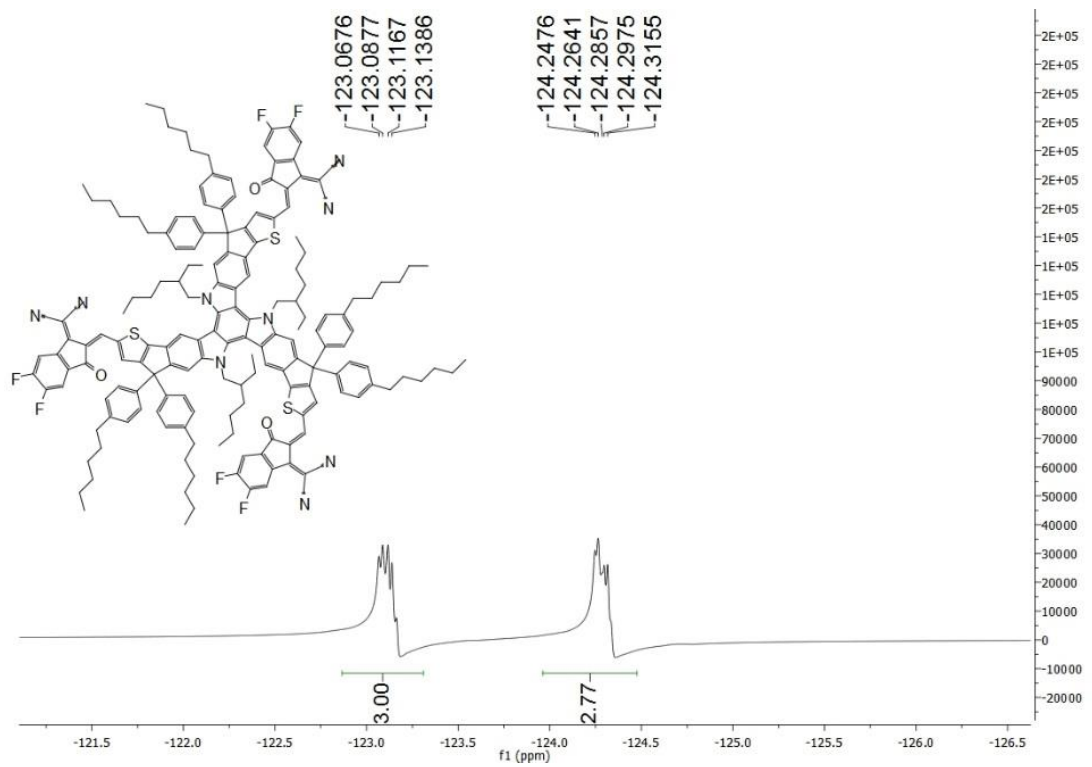

Figure S29. The 19F NMR spectrum $(376 \mathrm{MHz})$ of TF1 in $\mathrm{CDCl}_{3}$. 


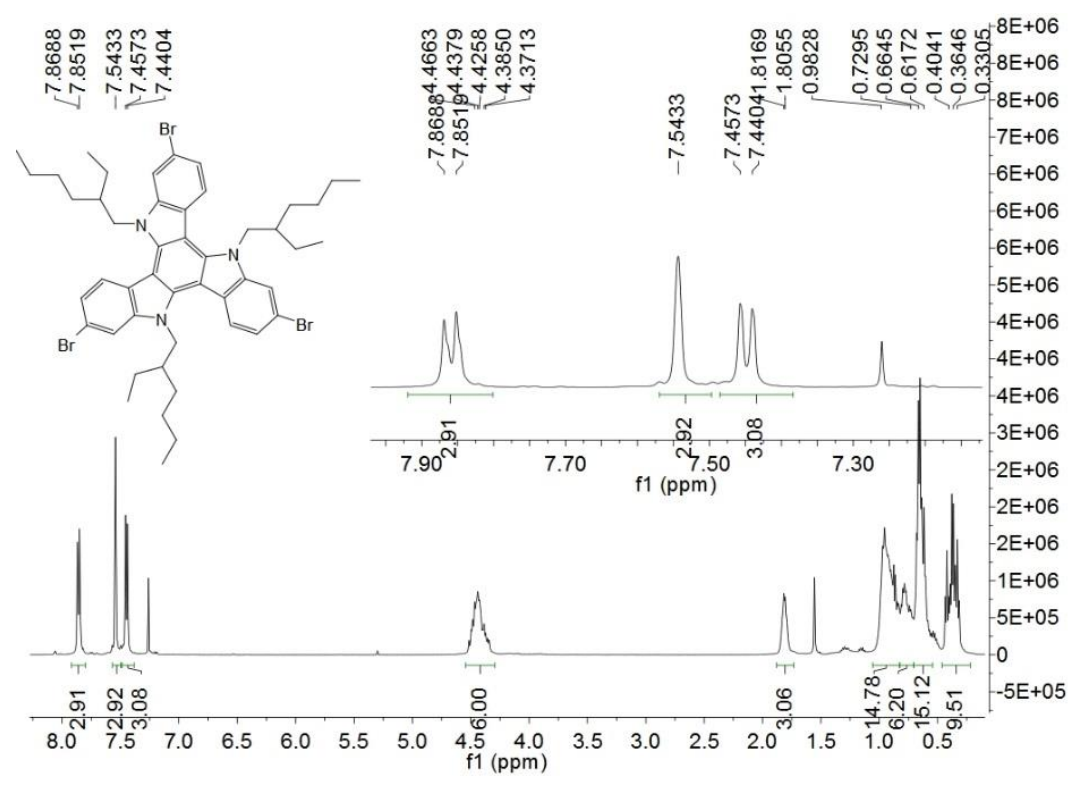

Figure S30. The ${ }^{1} \mathrm{H}$ NMR spectrum $(500 \mathrm{MHz})$ of $\mathbf{8}$ in $\mathrm{CDCl}_{3}$. 


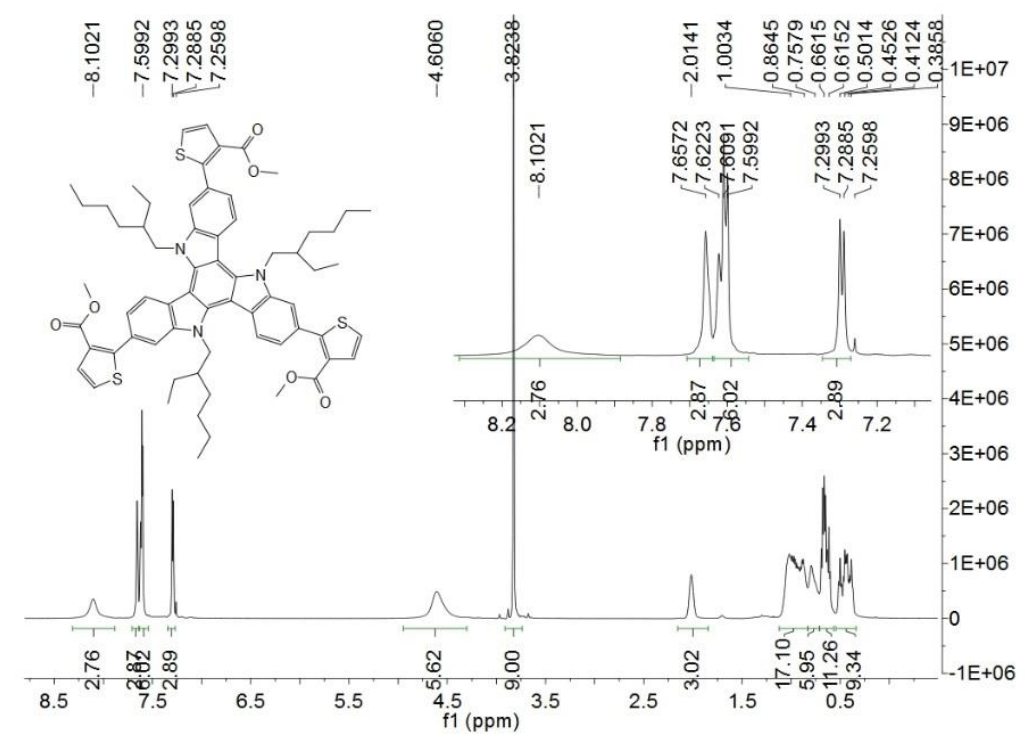

Figure S31. The ${ }^{1} \mathrm{H}$ NMR spectrum $(500 \mathrm{MHz})$ of 9 in $\mathrm{CDCl}_{3}$. 


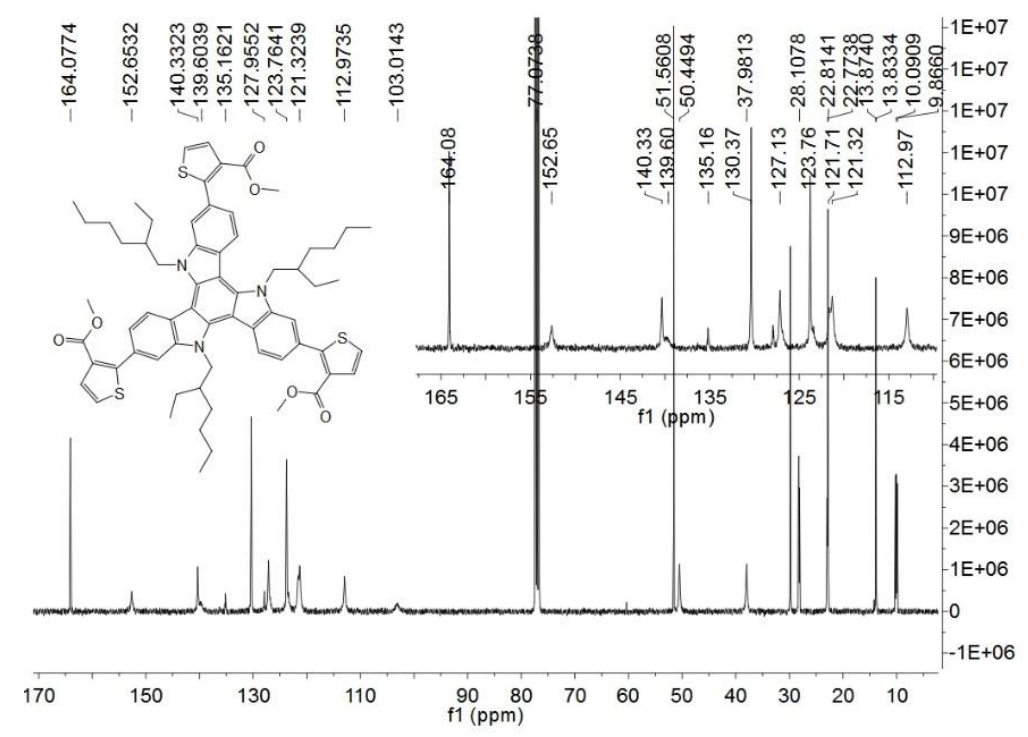

Figure S32. The ${ }^{13} \mathrm{C}$ NMR spectrum $(125 \mathrm{MHz})$ of 9 in $\mathrm{CDCl}_{3}$. 


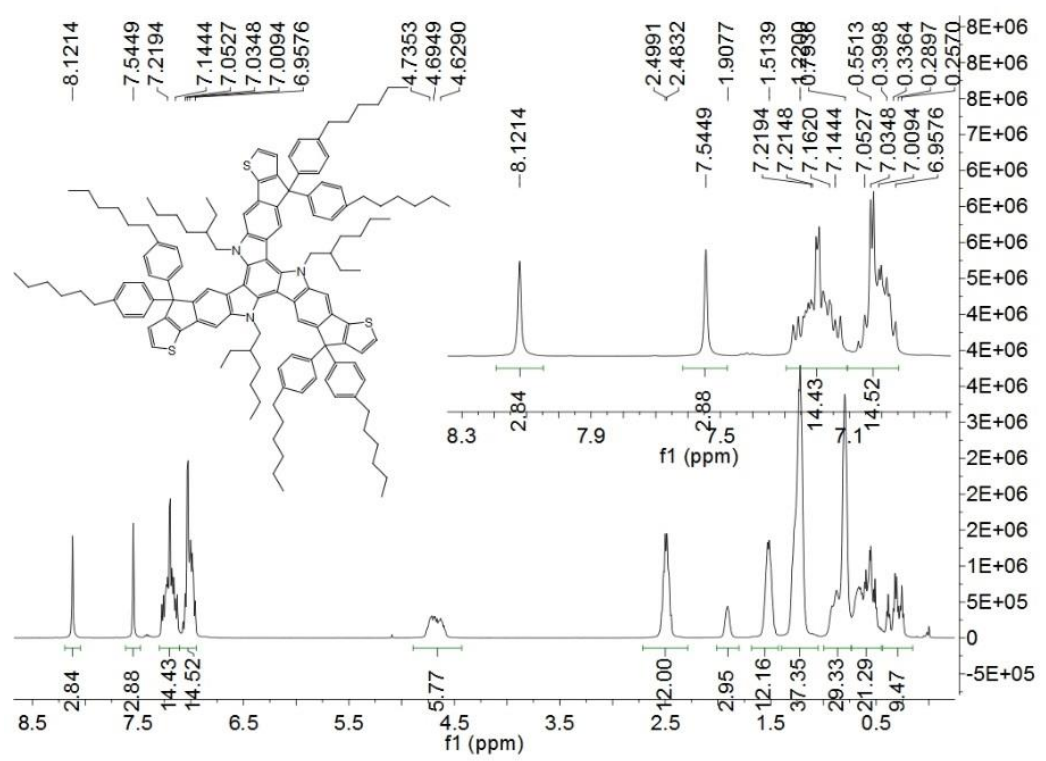

Figure S33. The ${ }^{1} \mathrm{H}$ NMR spectrum $(500 \mathrm{MHz})$ of $\mathbf{T} 2$ in $\mathrm{CDCl}_{3}$. 


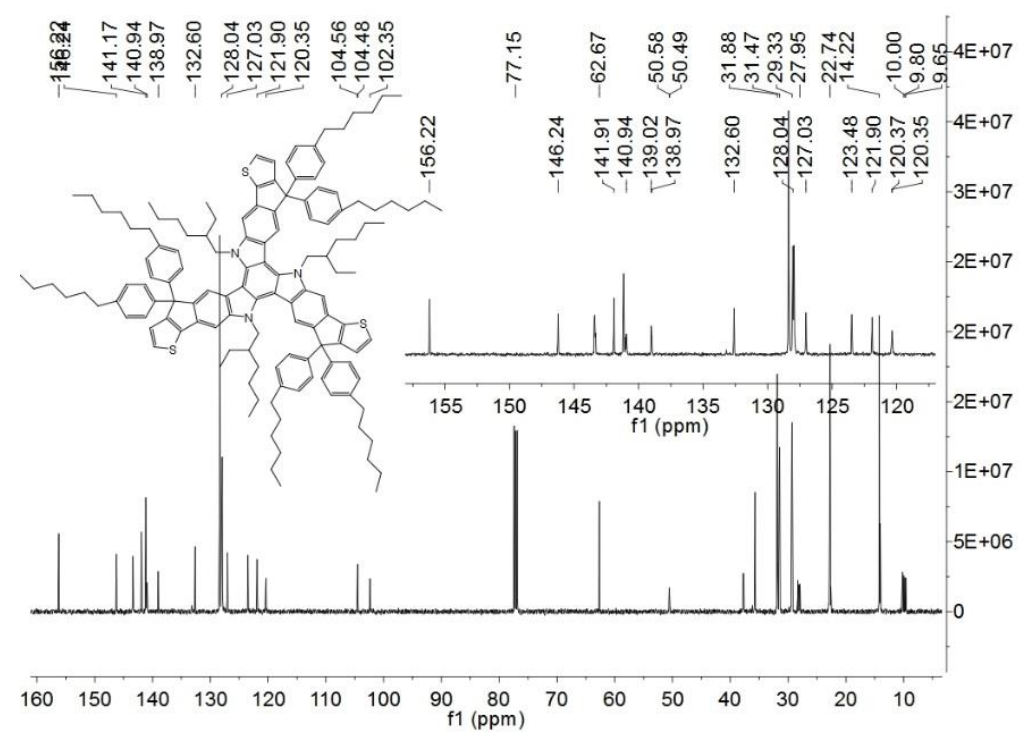

Figure S34. The ${ }^{13} \mathrm{C}$ NMR spectrum $(125 \mathrm{MHz})$ of $\mathbf{T} 2$ in $\mathrm{CDCl}_{3}$. 


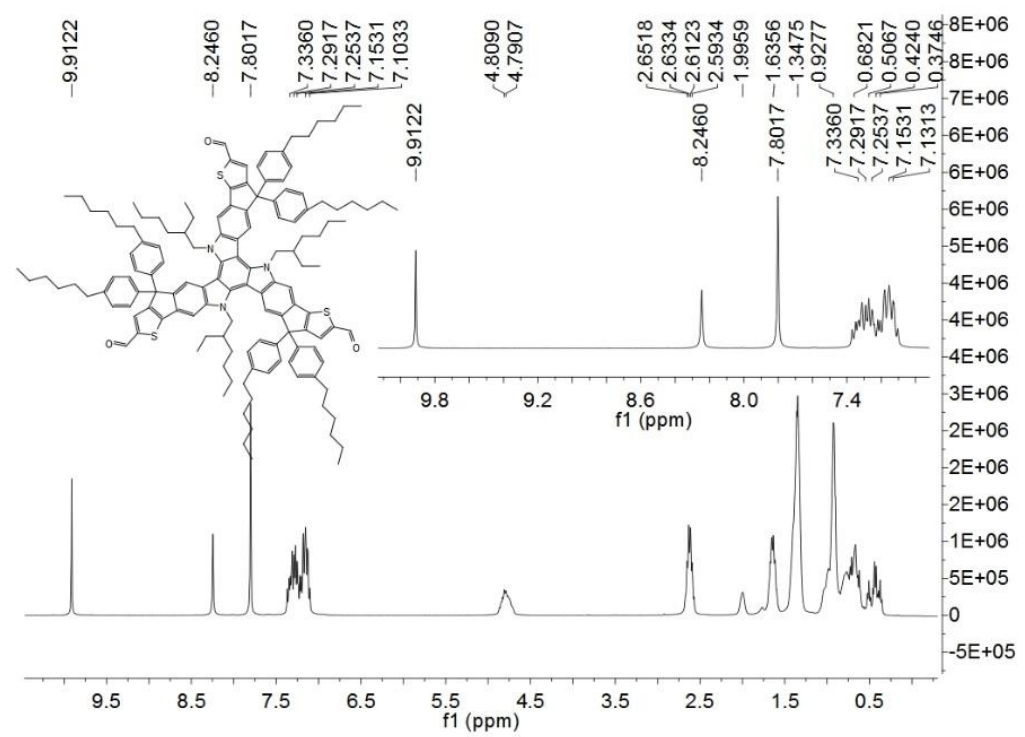

Figure S35. The ${ }^{1} \mathrm{H}$ NMR spectrum $(400 \mathrm{MHz})$ of $\mathbf{1 6}$ in $\mathrm{CDCl}_{3}$. 


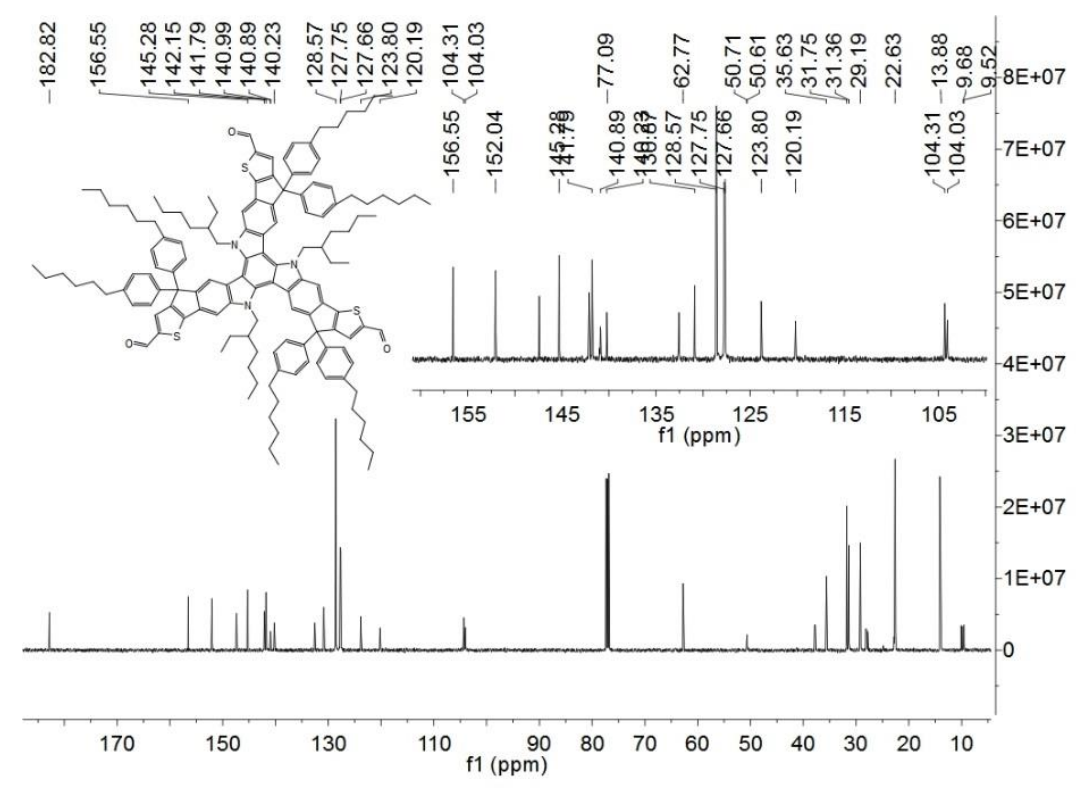

Figure S36. The ${ }^{13} \mathrm{C}$ NMR spectrum $(125 \mathrm{MHz})$ of 16 in $\mathrm{CDCl}_{3}$. 


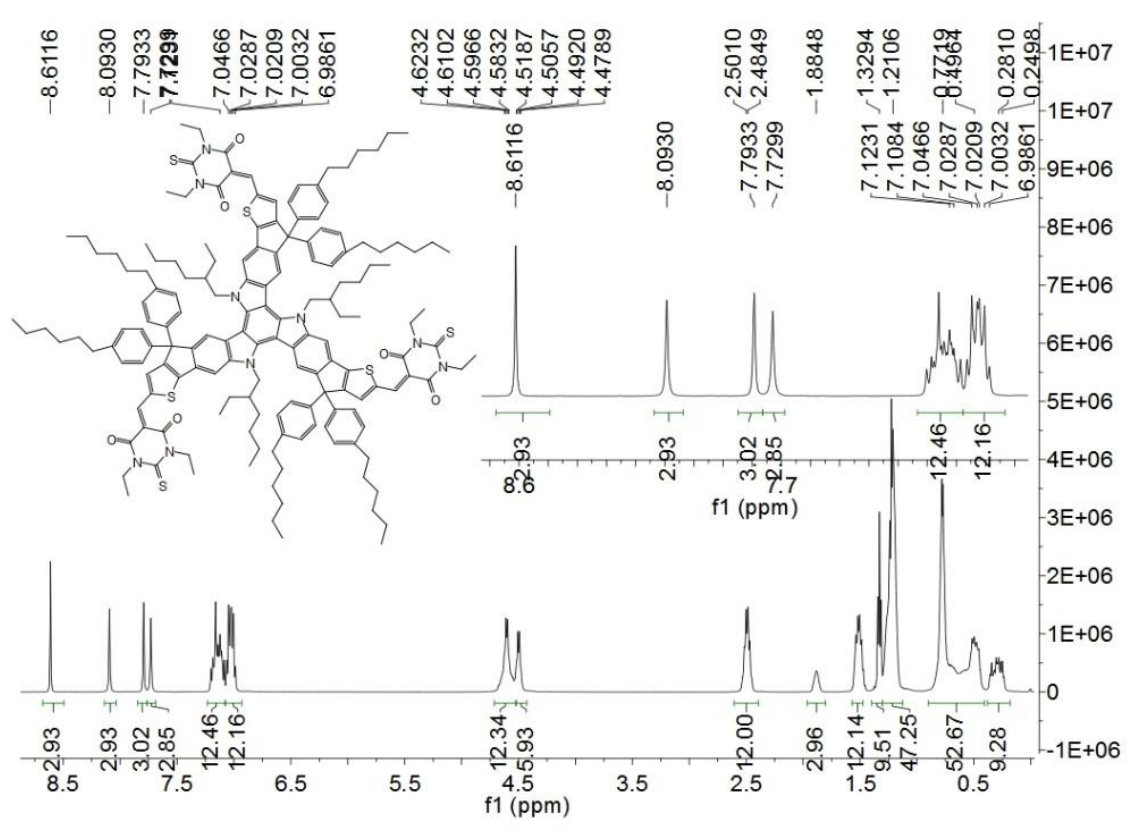

Figure S37. The ${ }^{1} \mathrm{H}$ NMR spectrum $(500 \mathrm{MHz})$ of $\mathbf{T B 2}$ in $\mathrm{CDCl}_{3}$. 


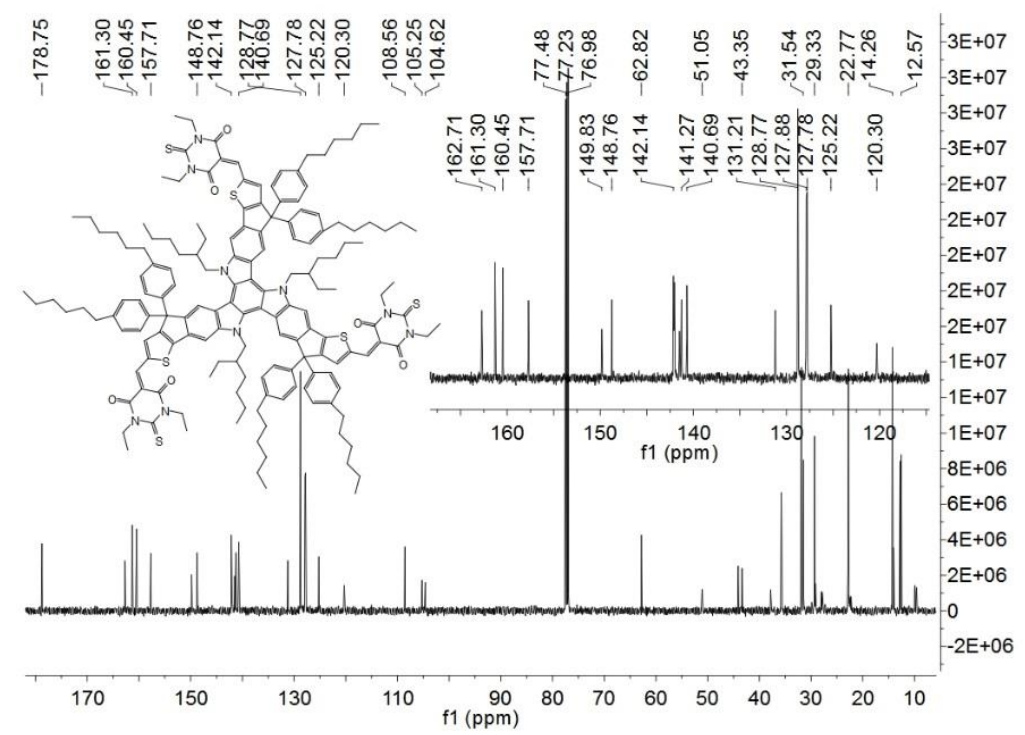

Figure S38. The ${ }^{13} \mathrm{C}$ NMR spectrum $(125 \mathrm{MHz})$ of $\mathbf{T B 2}$ in $\mathrm{CDCl}_{3}$. 


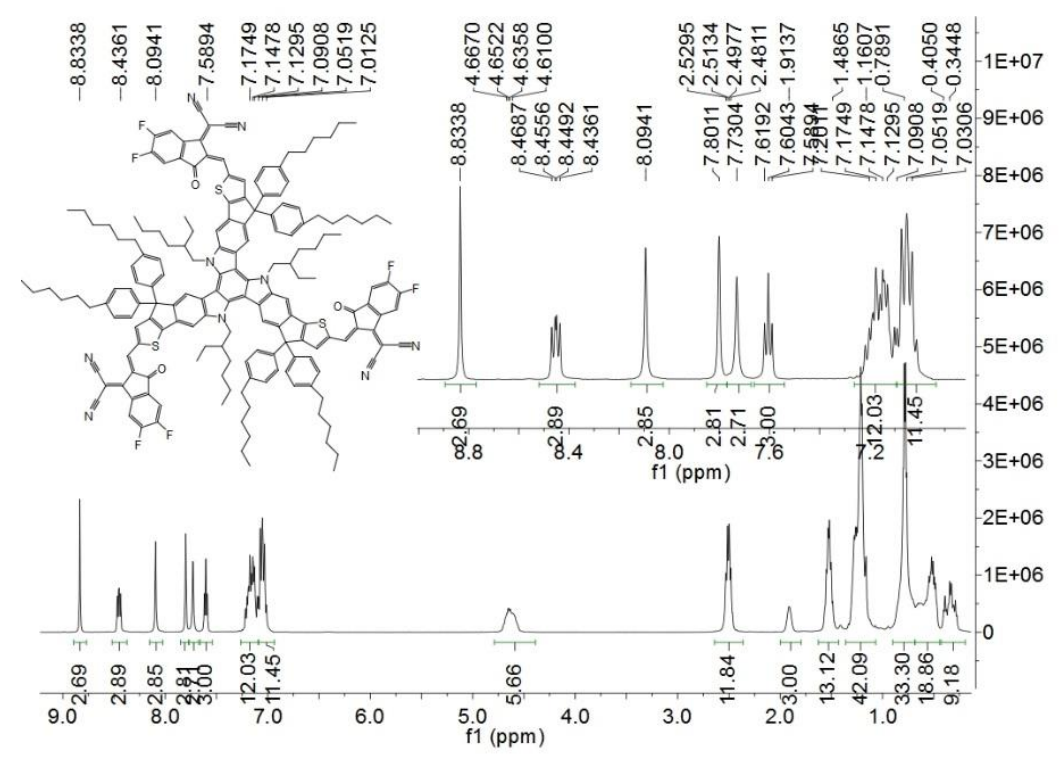

Figure S39. The ${ }^{1} \mathrm{H}$ NMR spectrum $(500 \mathrm{MHz})$ of TF2 in $\mathrm{CDCl}_{3}$. 


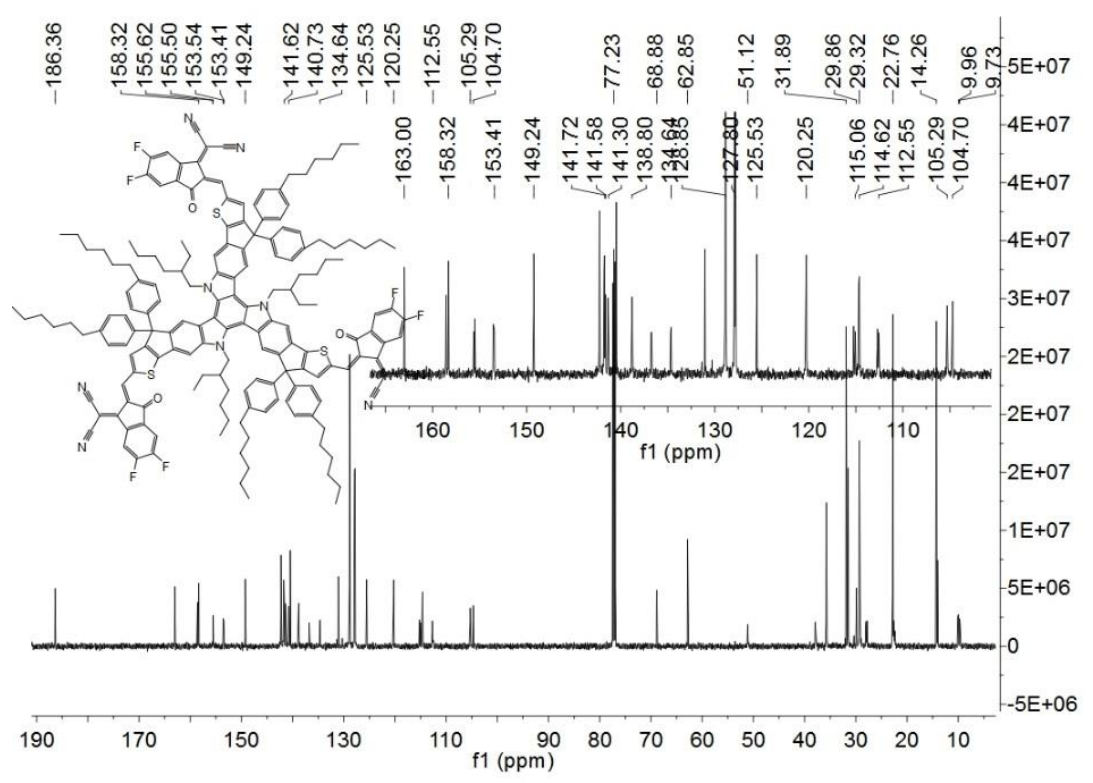

Figure S40. The ${ }^{13} \mathrm{C}$ NMR spectrum $(125 \mathrm{MHz})$ of $\mathbf{T F 2}$ in $\mathrm{CDCl}_{3}$. 


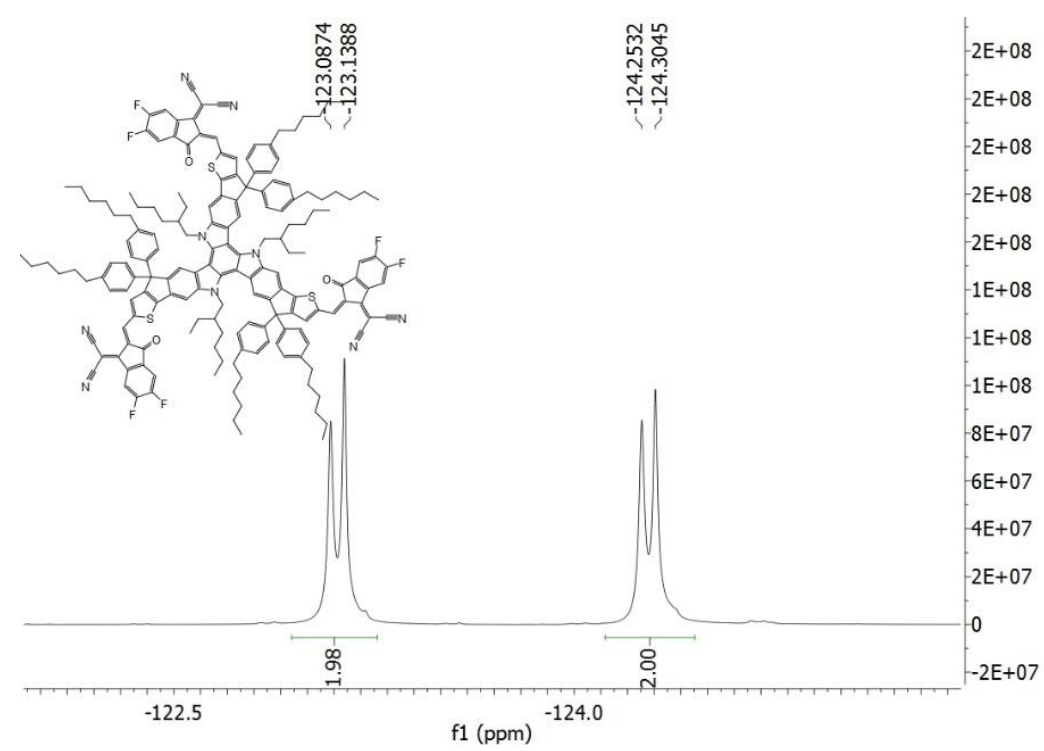

Figure S41. The $19 \mathrm{~F}\{1 \mathrm{H}\}$ NMR spectrum $(376 \mathrm{MHz})$ of TF2 in $\mathrm{CDCl}_{3}$. 


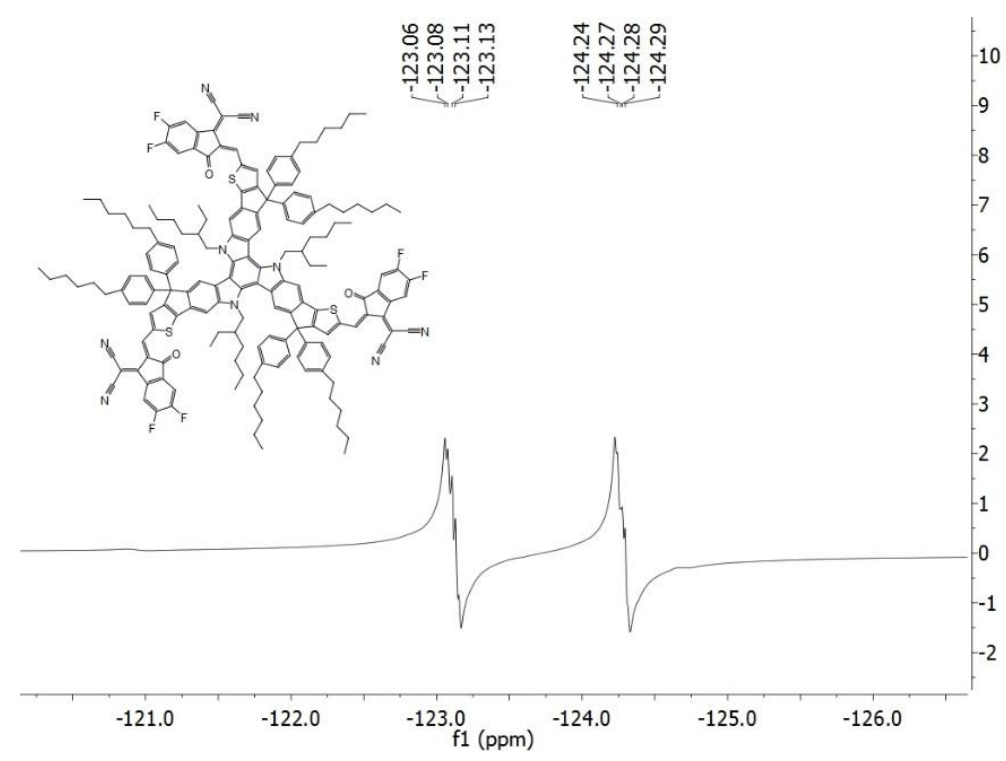

Figure S42. The 19F NMR spectrum $(376 \mathrm{MHz})$ of $\mathbf{T F 2}$ in $\mathrm{CDCl}_{3}$. 


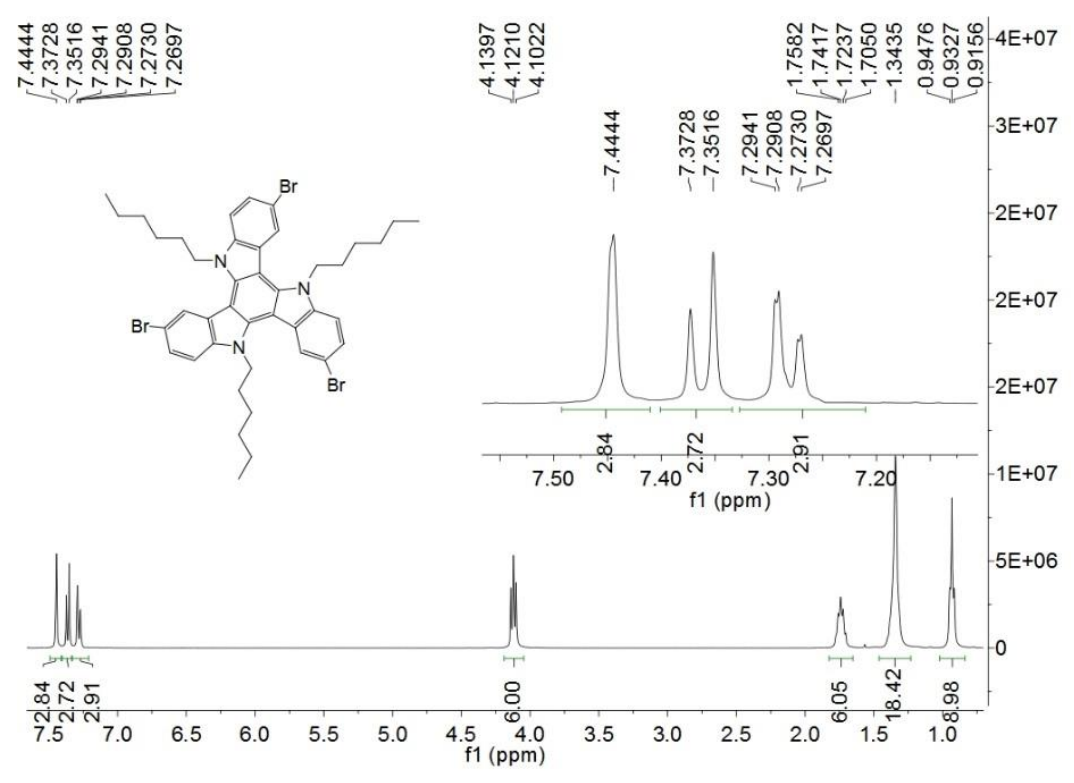

Figure S43. The ${ }^{1} \mathrm{H}$ NMR spectrum $(400 \mathrm{MHz})$ of 12 in $\mathrm{CDCl}_{3}$. 


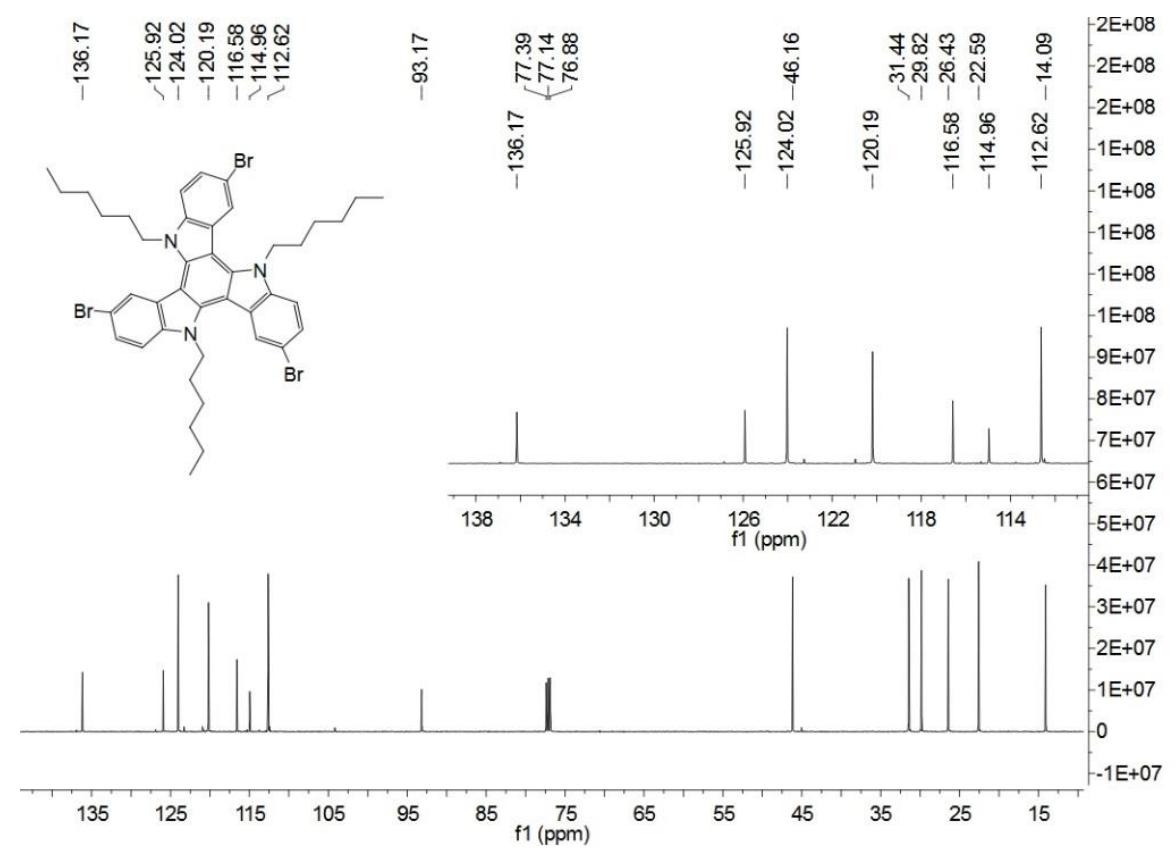

Figure S44. The ${ }^{13} \mathrm{C}$ NMR spectrum $(125 \mathrm{MHz})$ of 12 in $\mathrm{CDCl}_{3}$. 


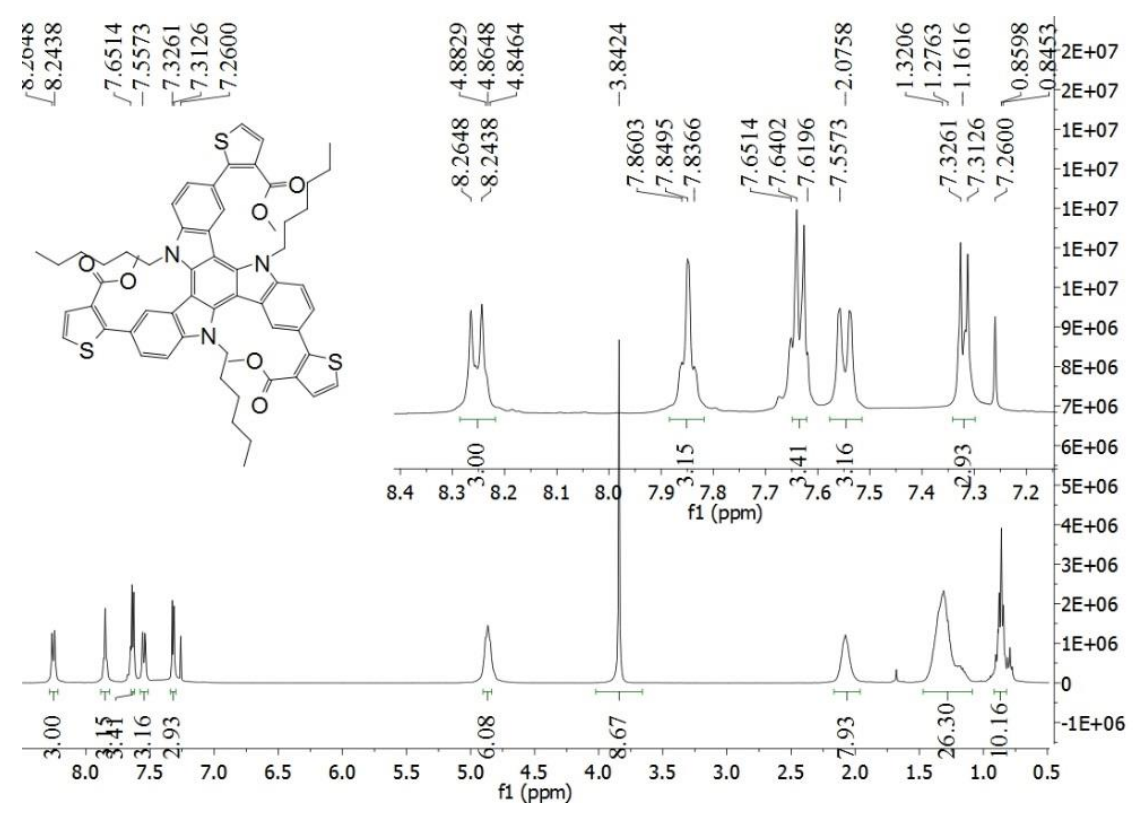

Figure $\mathbf{S 4 5}$. The ${ }^{1} \mathrm{H}$ NMR spectrum $(400 \mathrm{MHz})$ of 13 in $\mathrm{CDCl}_{3}$. 


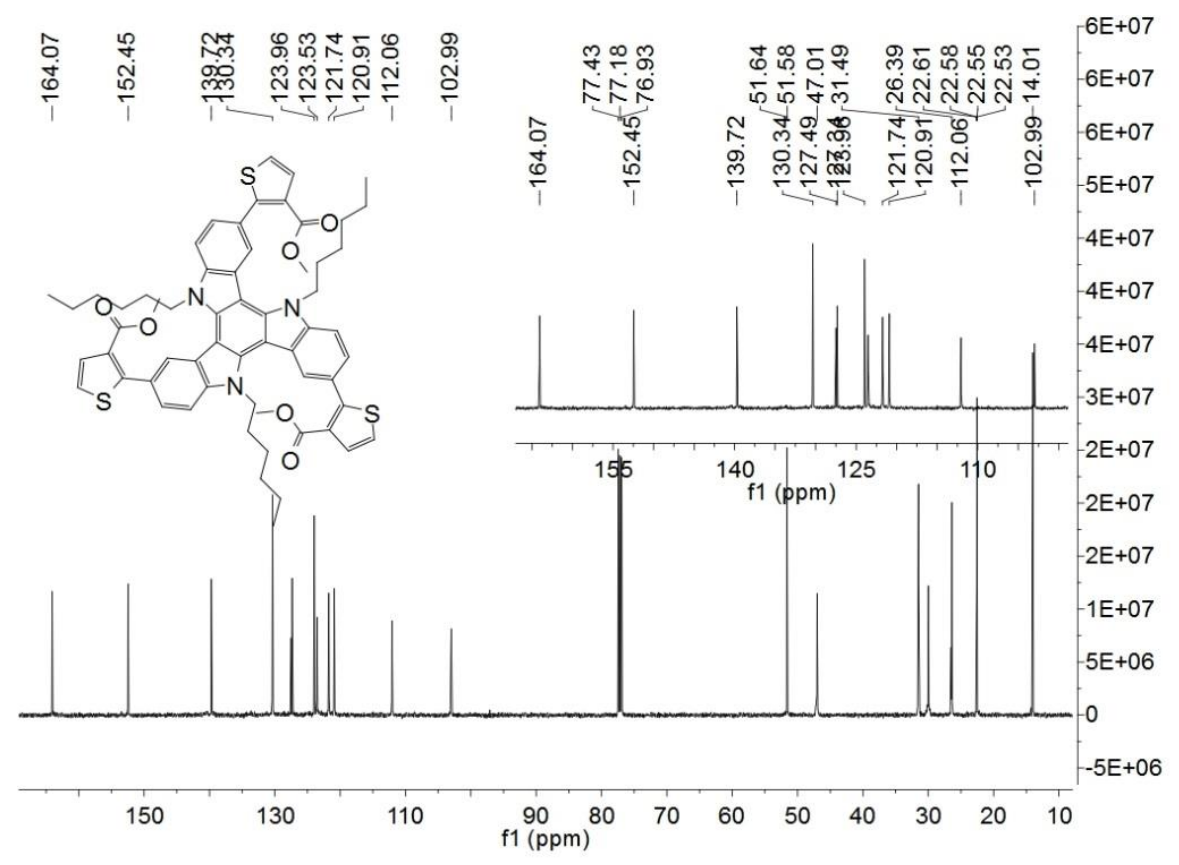

Figure S46. The ${ }^{13} \mathrm{C}$ NMR spectrum $(125 \mathrm{MHz})$ of 13 in $\mathrm{CDCl}_{3}$. 


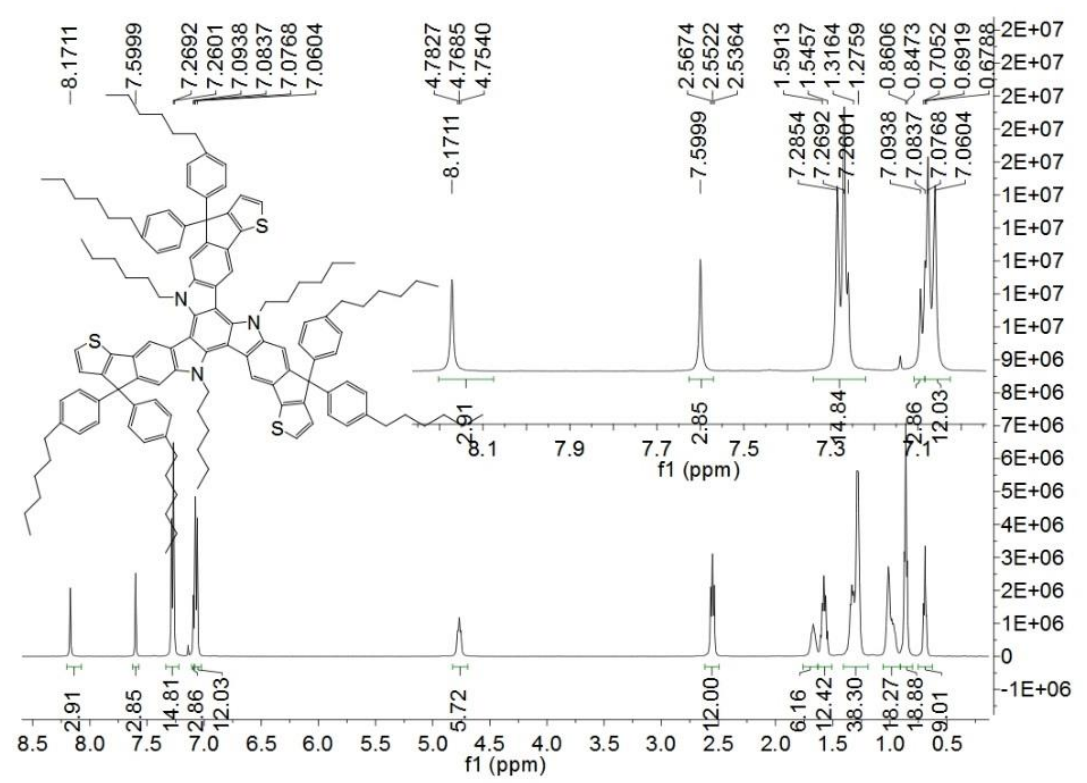

Figure S47. The ${ }^{1} \mathrm{H}$ NMR spectrum $(500 \mathrm{MHz})$ of T1-C6 in $\mathrm{CDCl}_{3}$. 


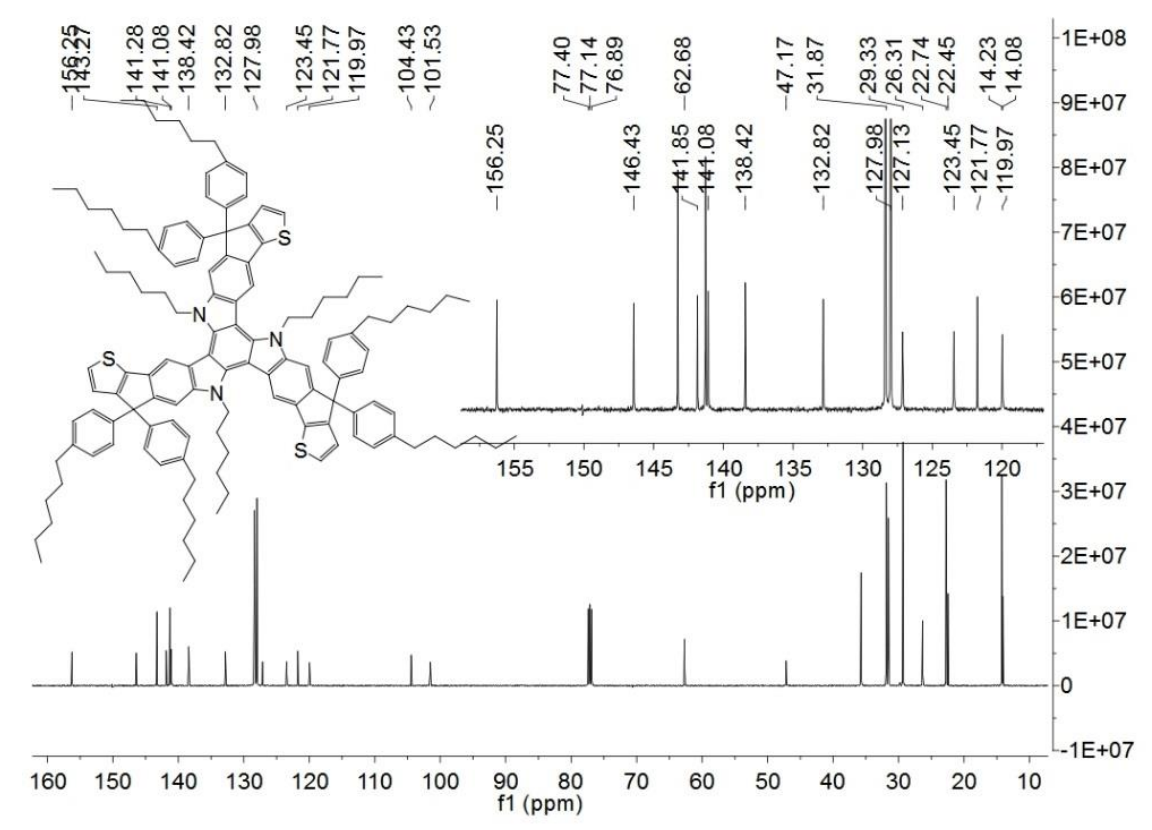

Figure S48. The ${ }^{13} \mathrm{C}$ NMR spectrum $(125 \mathrm{MHz})$ of T1-C6 in $\mathrm{CDCl}_{3}$. 


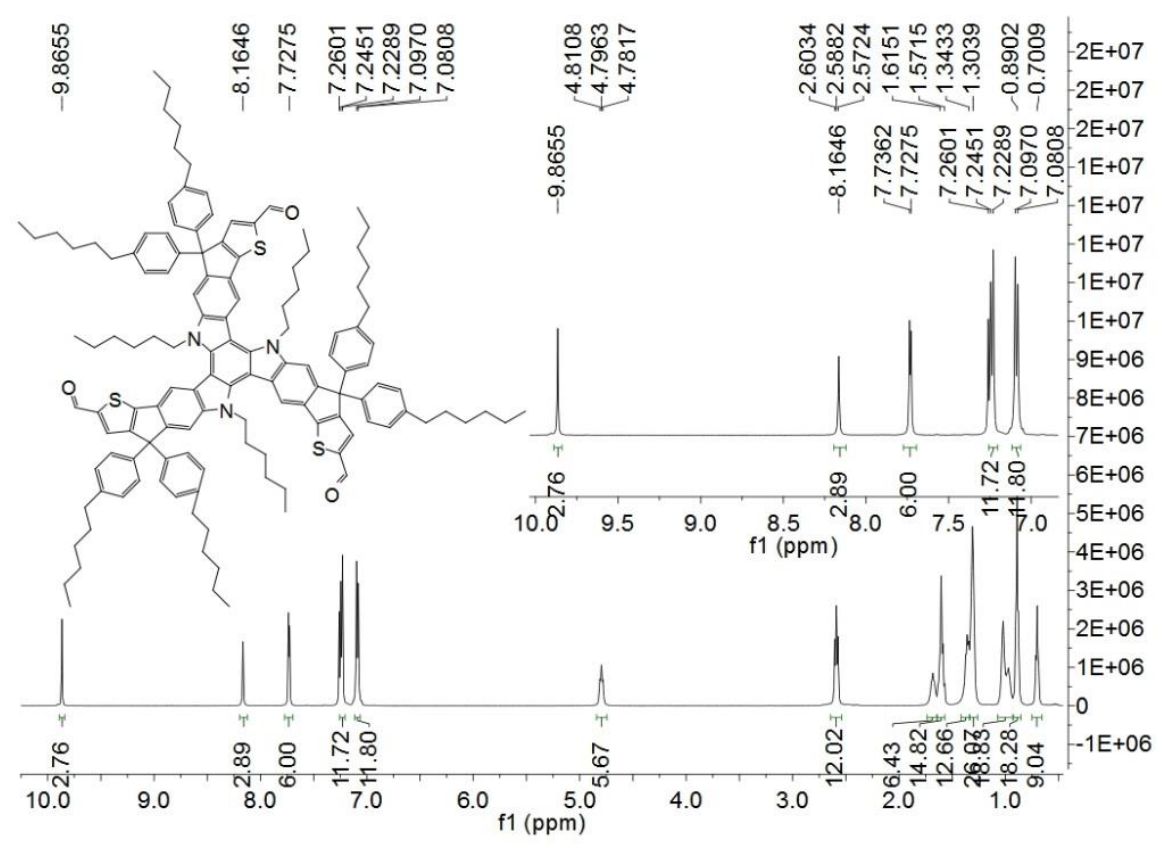

Figure S49. The ${ }^{1} \mathrm{H}$ NMR spectrum $(500 \mathrm{MHz})$ of 14 in $\mathrm{CDCl}_{3}$. 


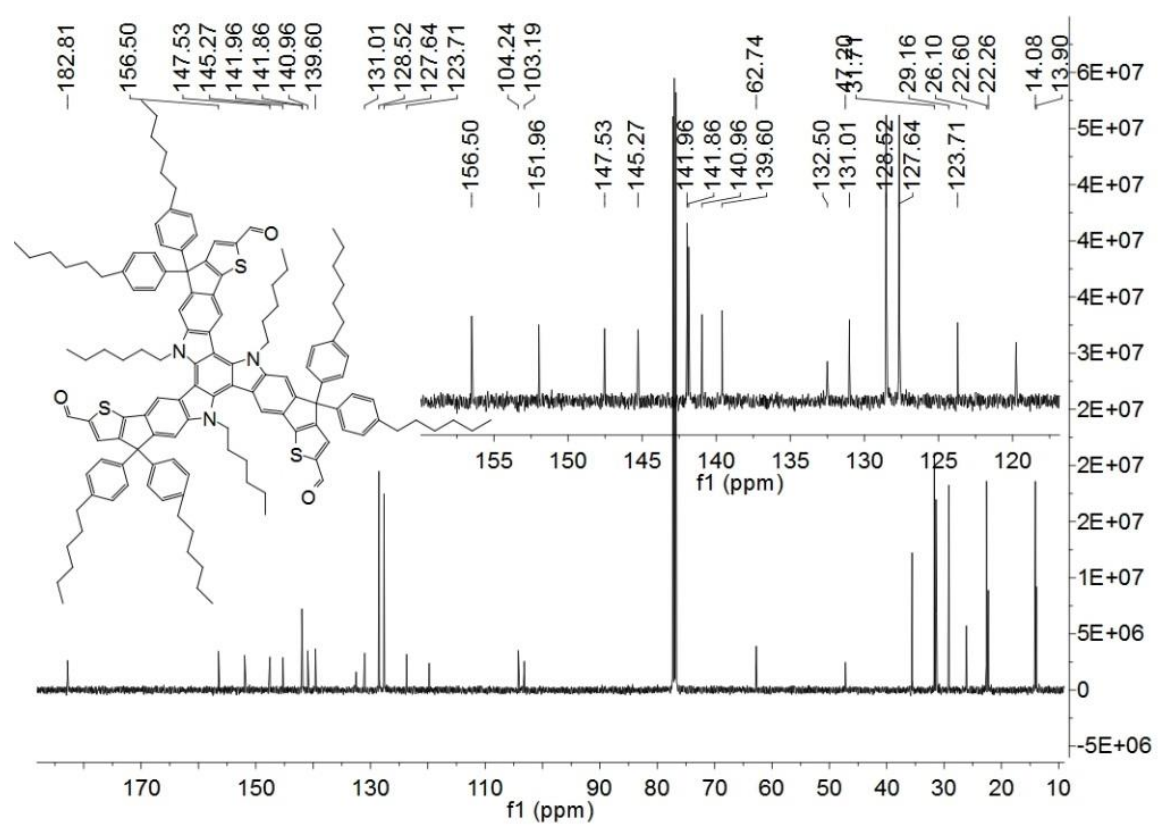

Figure S50. The ${ }^{13} \mathrm{C}$ NMR spectrum $(125 \mathrm{MHz})$ of 14 in $\mathrm{CDCl}_{3}$. 


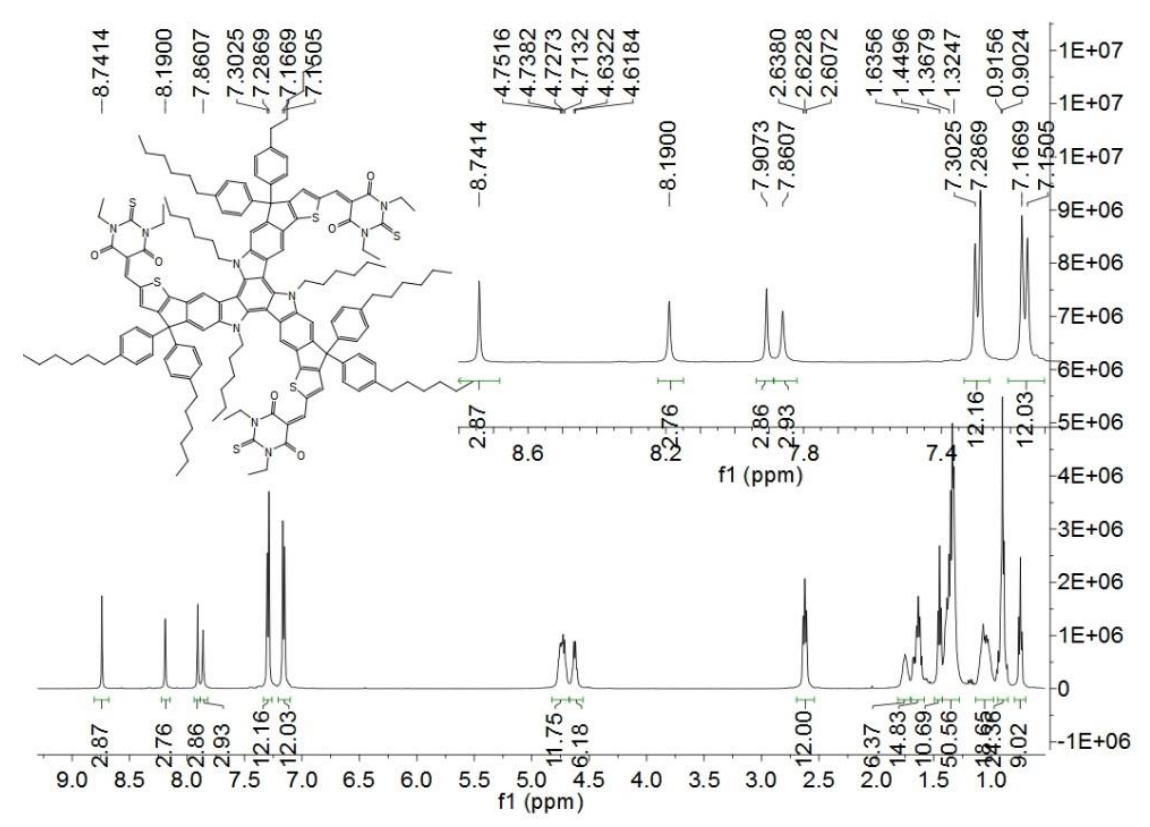

Figure S51. The ${ }^{1} \mathrm{H}$ NMR spectrum $(500 \mathrm{MHz})$ of TB1-C6 in $\mathrm{CDCl}_{3}$. 


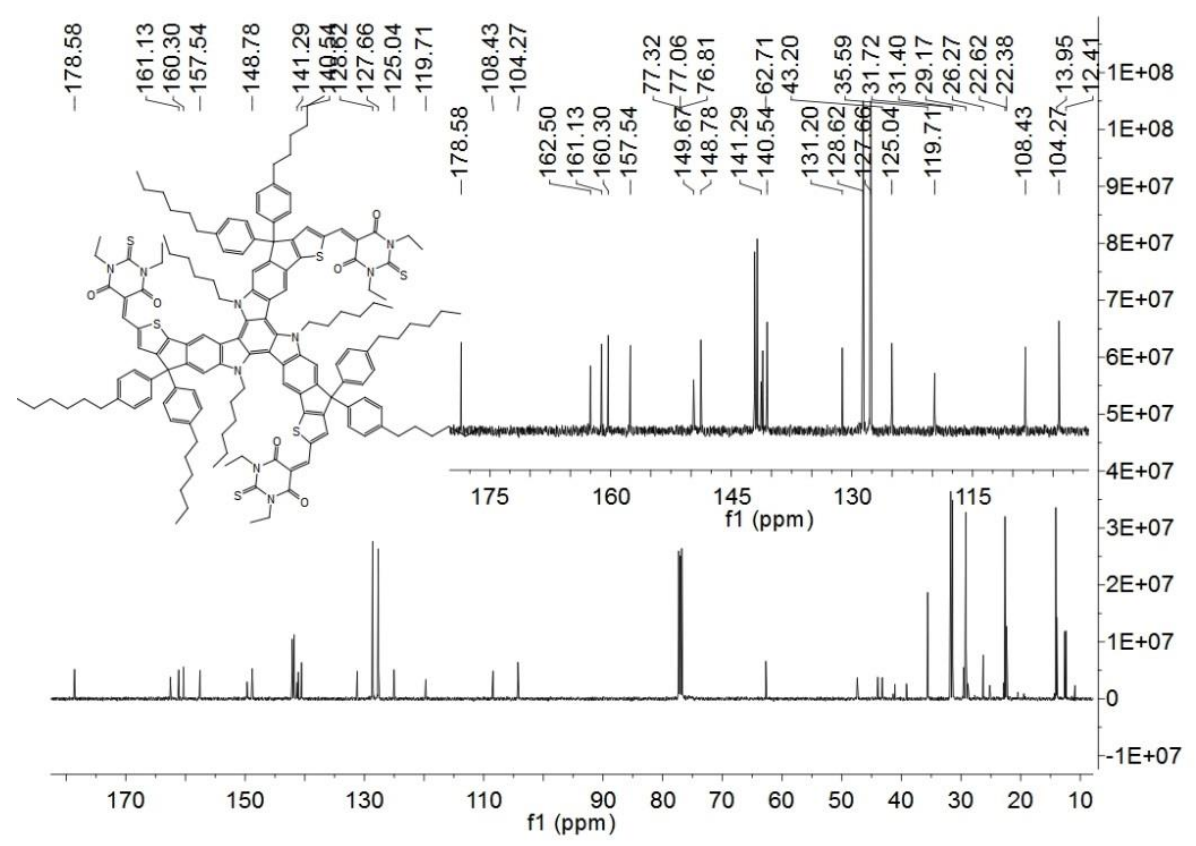

Figure S52. The ${ }^{13} \mathrm{C}$ NMR spectrum $(125 \mathrm{MHz})$ of TB1-C6 in $\mathrm{CDCl}_{3}$. 


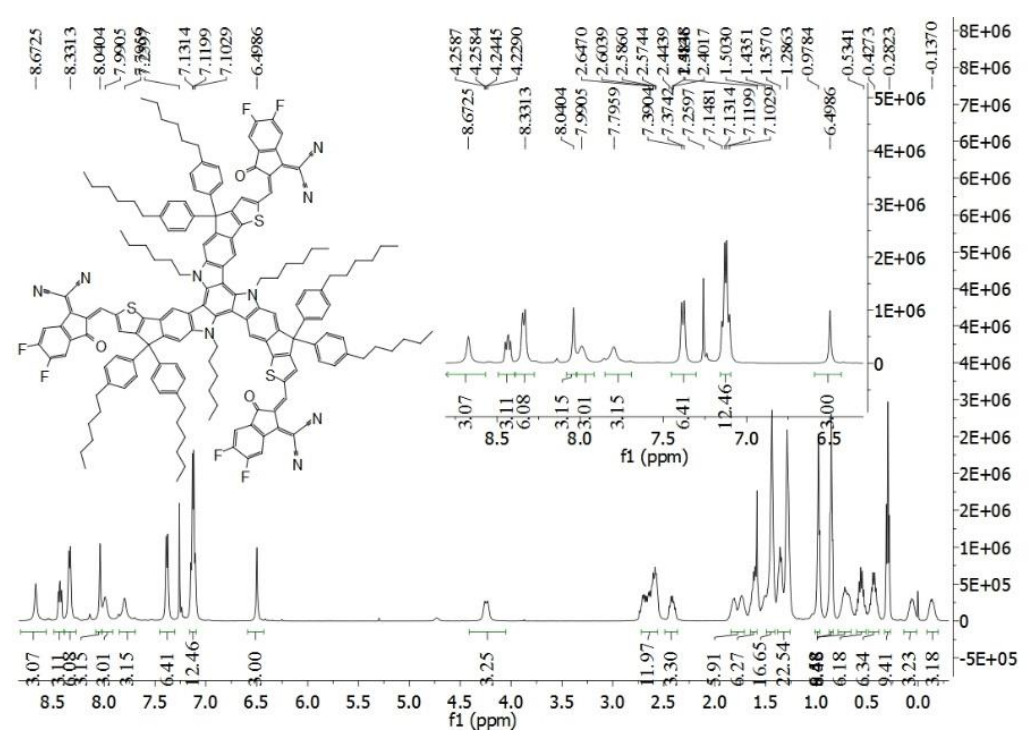

Figure S53. The ${ }^{1} \mathrm{H}$ NMR spectrum (500 MHz) of TF1-C6 in $\mathrm{CDCl}_{3}$. 


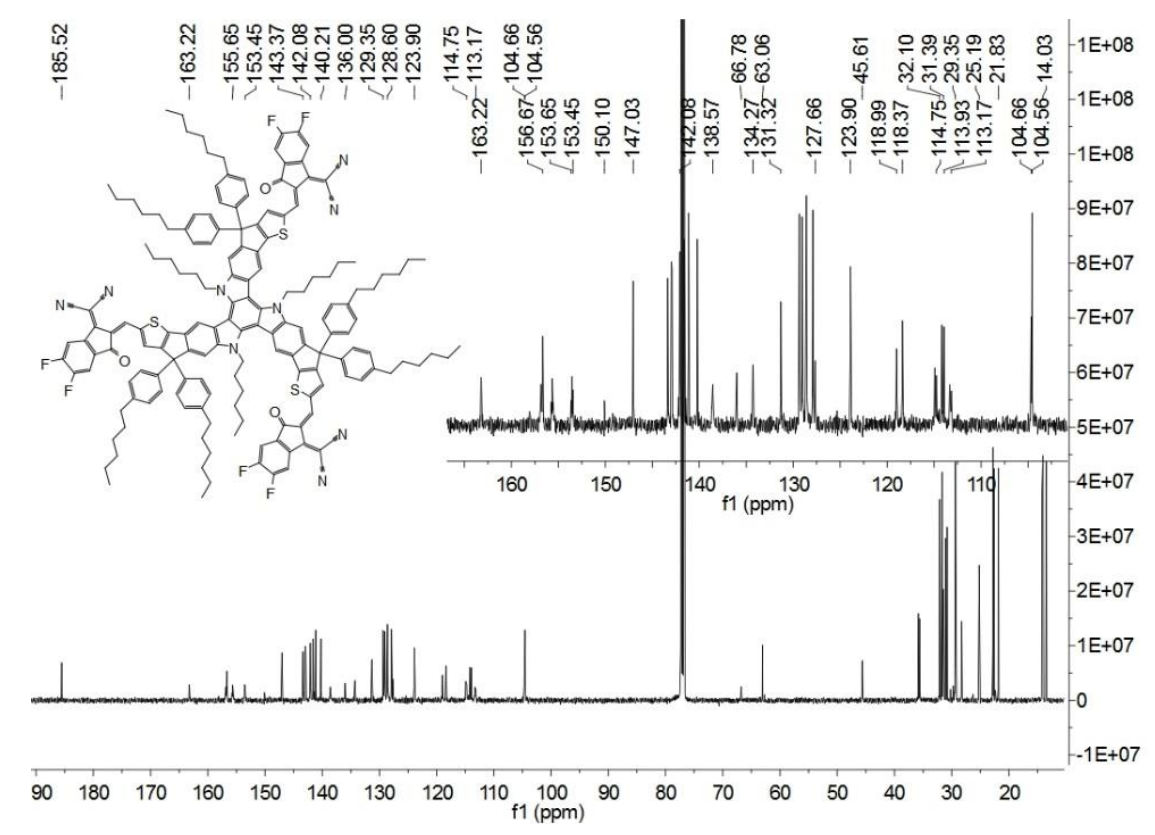

Figure S54. The ${ }^{13} \mathrm{C}$ NMR spectrum $(125 \mathrm{MHz})$ of TF1-C6 in $\mathrm{CDCl}_{3}$. 


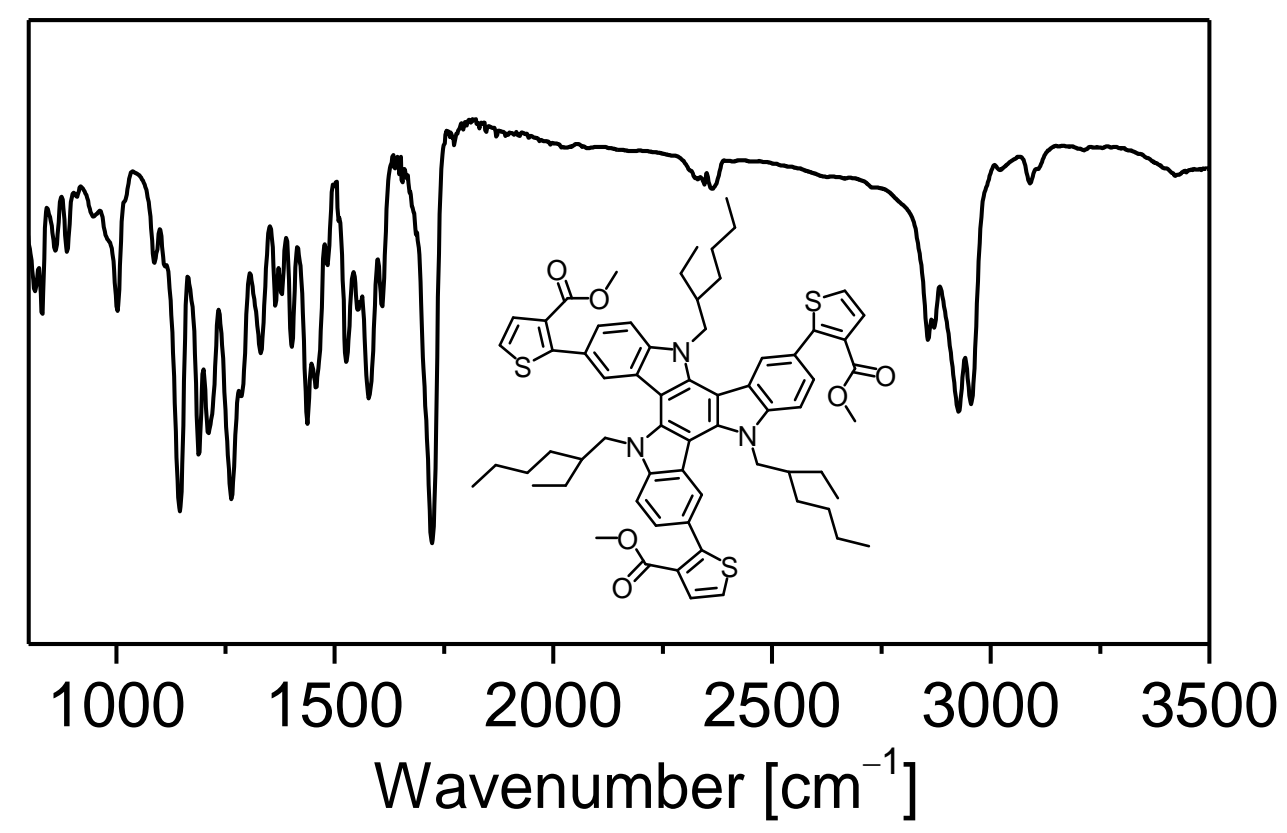

Figure S55. The IR spectrum (KBr) of compound 5. 


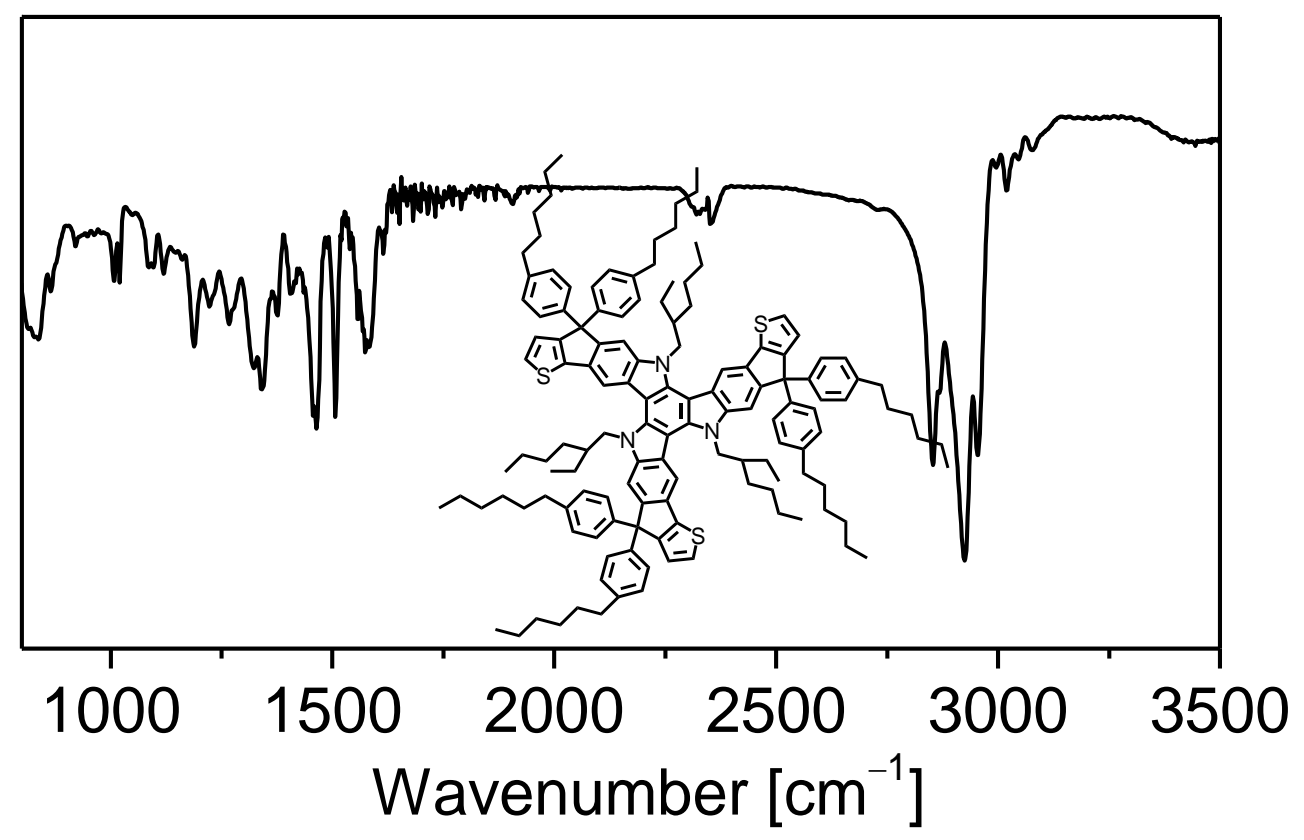

Figure S56. The IR spectrum (KBr) of compound T1. 


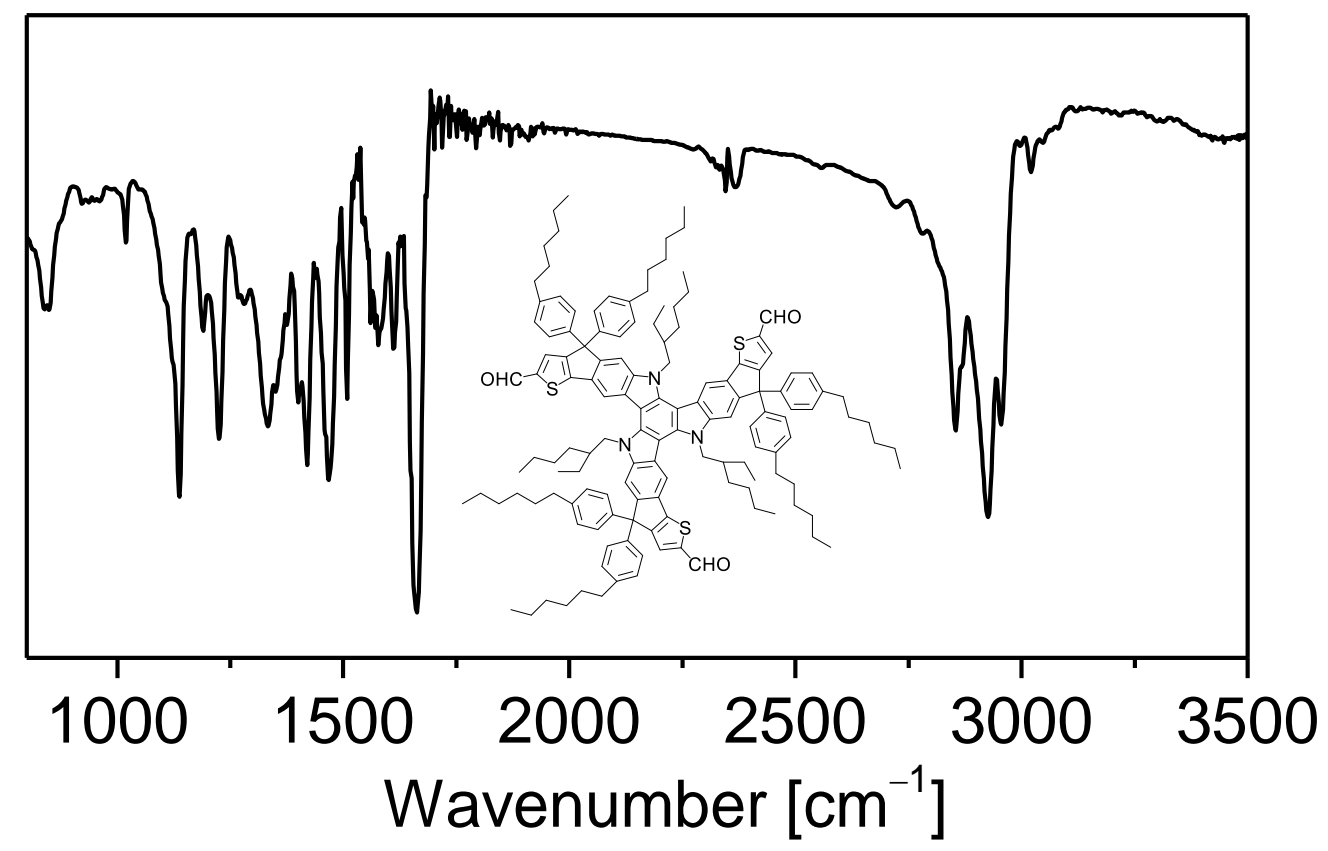

Figure S57. The IR spectrum (KBr) of compound 15. 


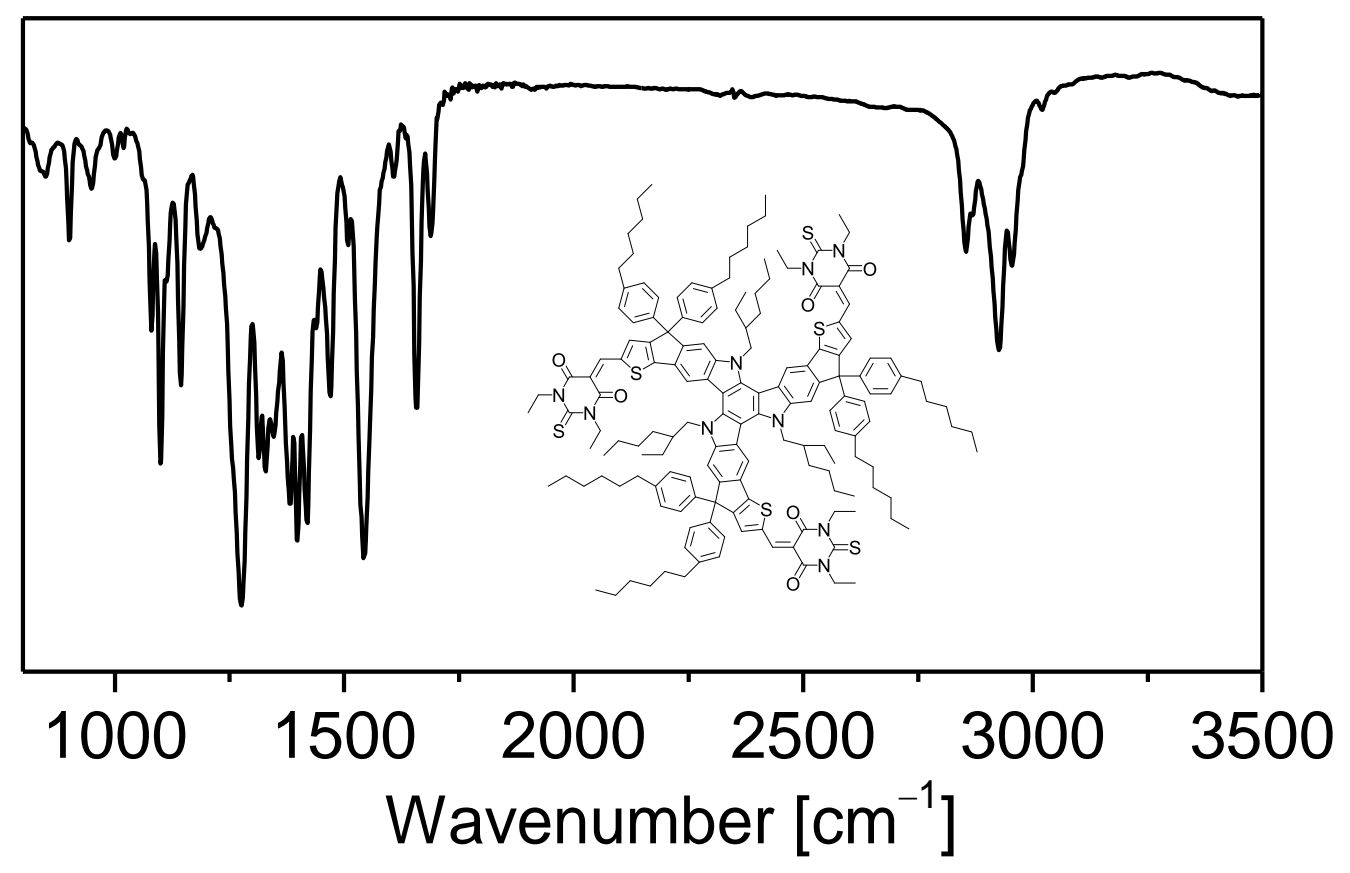

Figure S58. The IR spectrum (KBr) of compound TB1. 


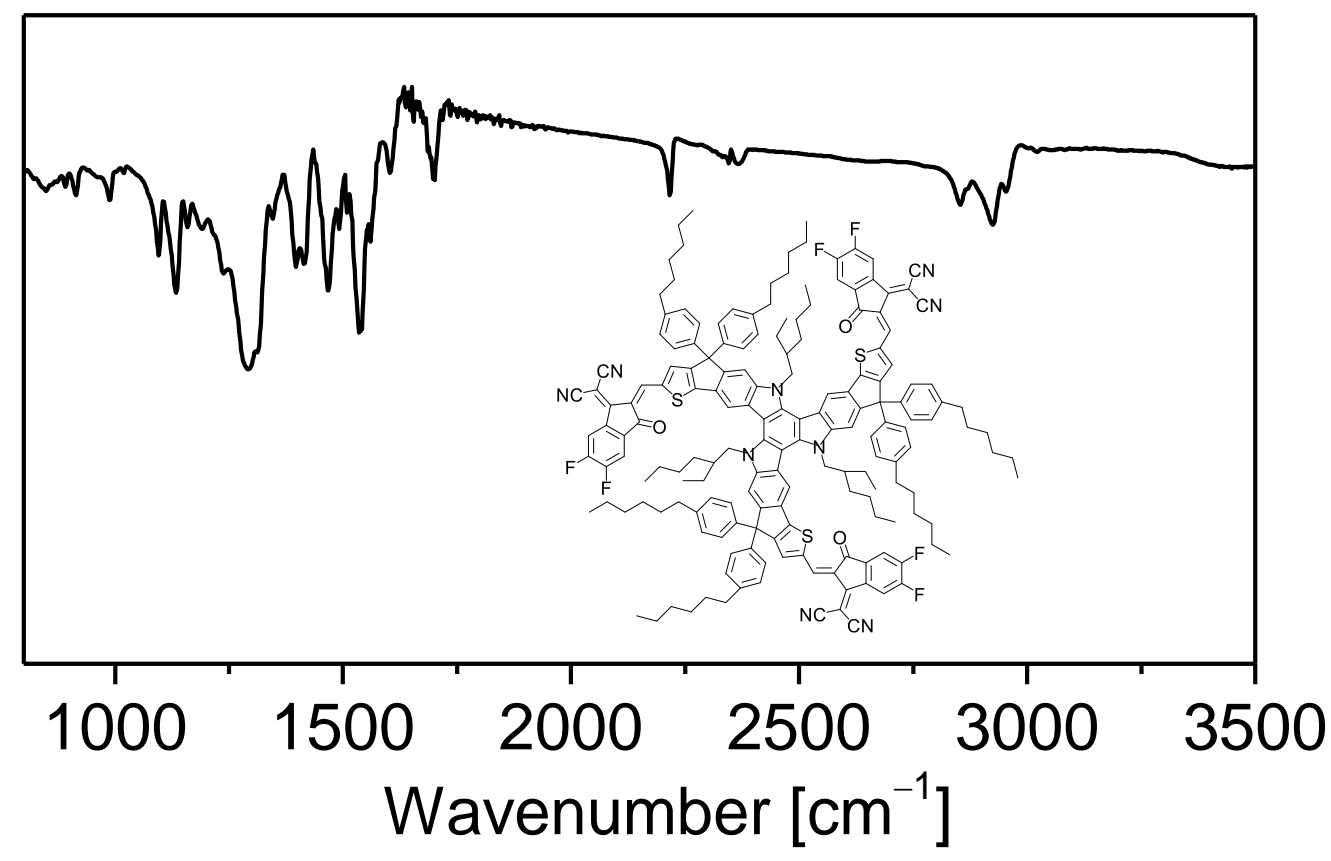

Figure S59. The IR spectrum (KBr) of compound TF1. 


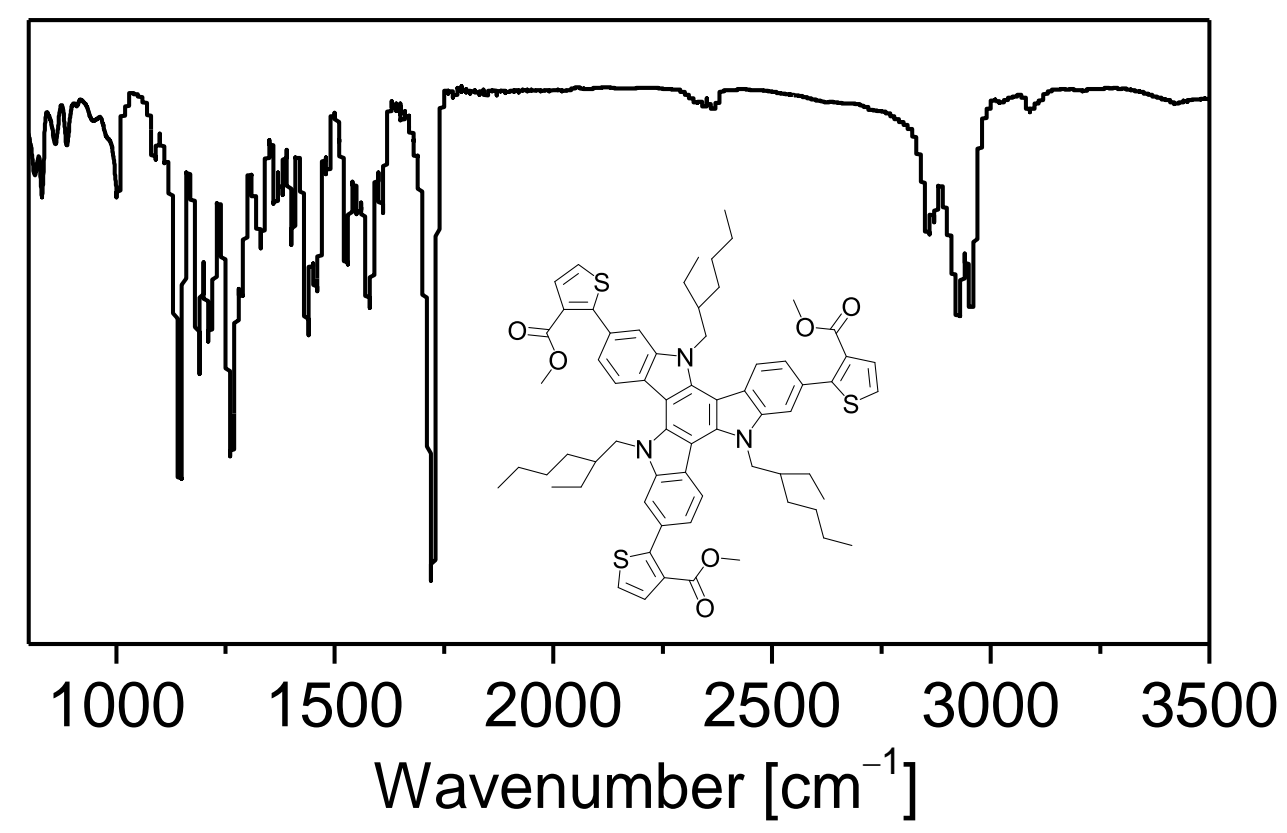

Figure S60. ATR-FTIR spectra of compound $\mathbf{1 0 .}$ 


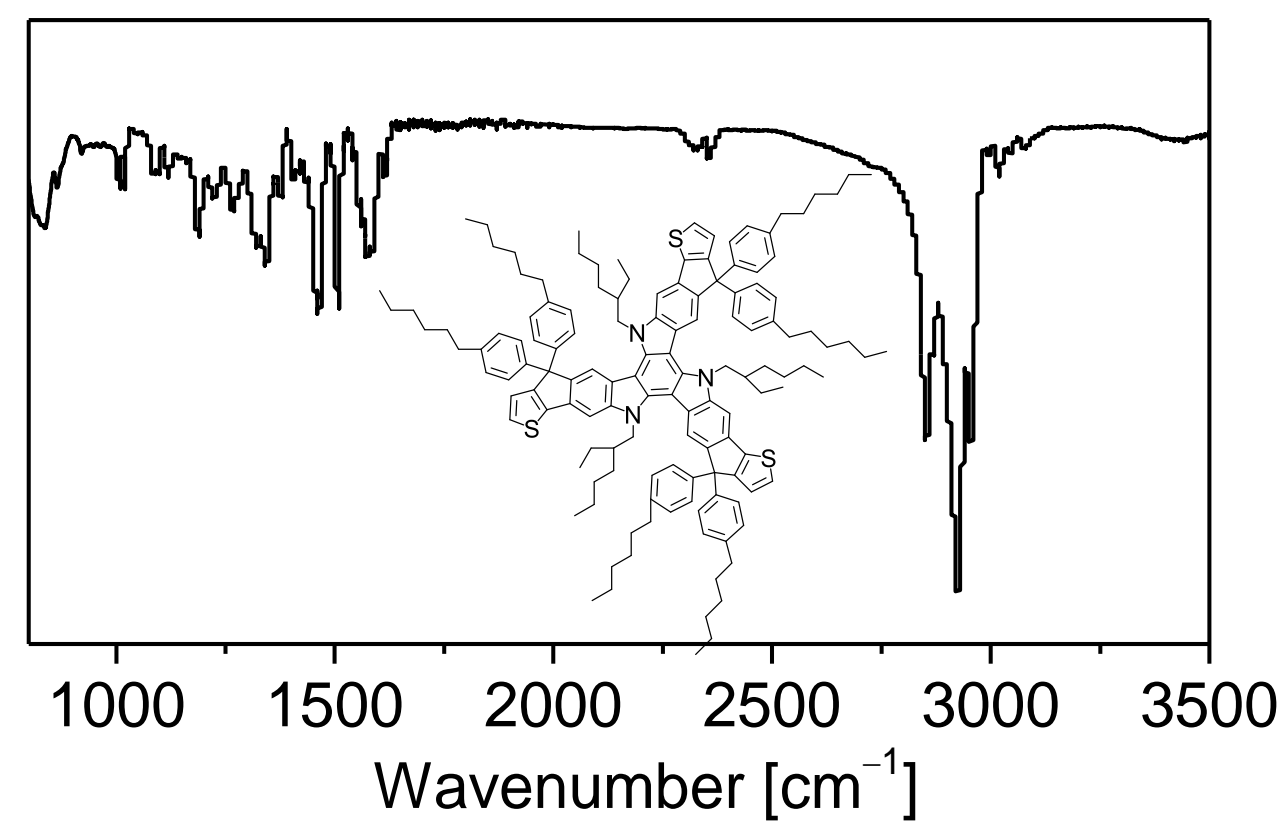

Figure S61. ATR-FTIR spectra of compound T2. 


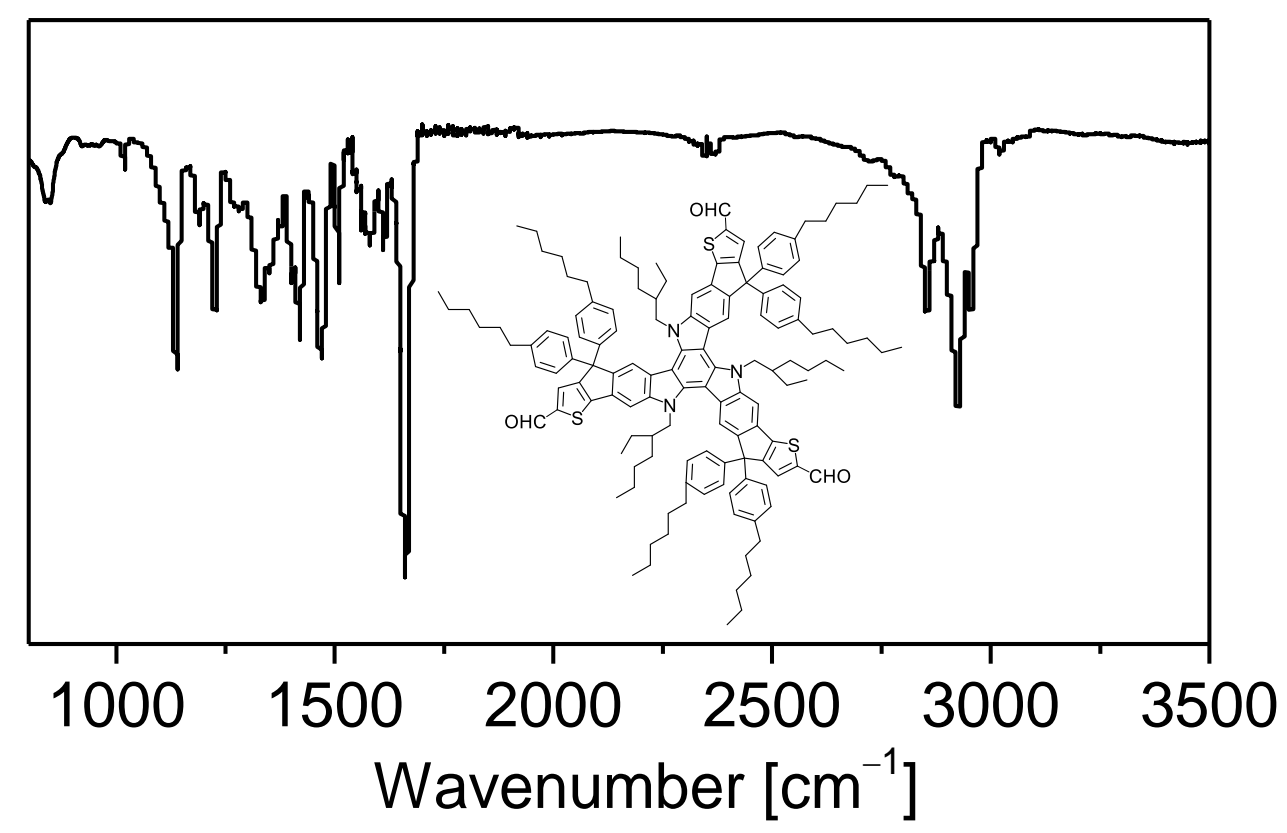

Figure S62. ATR-FTIR spectra of compound $\mathbf{1 6 .}$ 


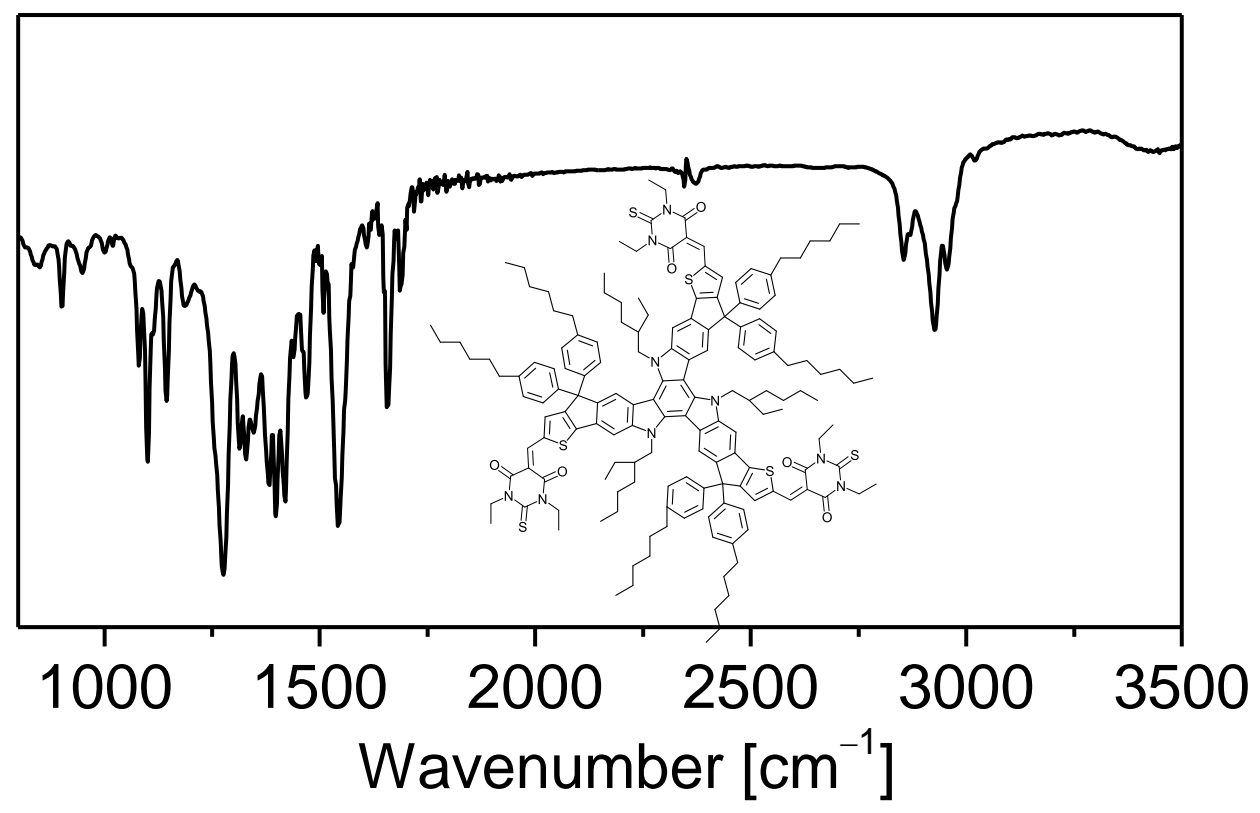

Figure S63. The IR spectrum (KBr) of compound TB2. 


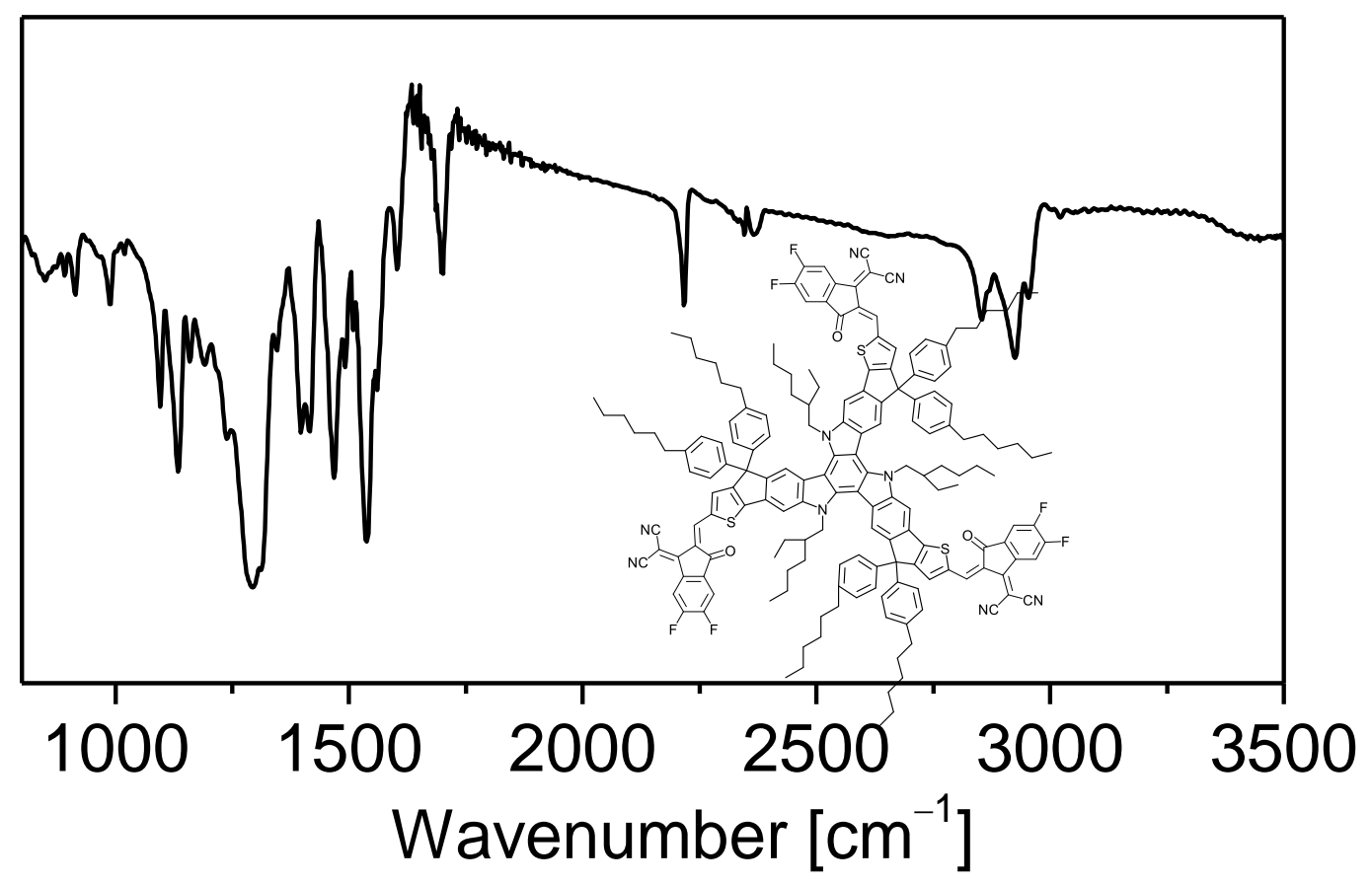

Figure S64. The IR spectrum (KBr) of compound TF2. 


\section{References}

(S1) Yao, H.; Cui, Y.; Yu, R.; Gao, B.; Zhang, H.; Hou, J. Design, Synthesis, and Photovoltaic Characterization of a Small Molecular Acceptor with an Ultra-Narrow Band Gap. Angew. Chem., Int. Ed. 2017, 56, 3045-3049.

(S2) Yin, C.; Lu, J.; Xu, Y.; Yun, Y.; Wang, K.; Li, J.; Jiang, L.; Sun, J.; Scully, A. D.; Huang, F.; Zhong, J.; Wang, J.; Cheng, Y.-B.; Qin, T.; Huang, W. Low-Cost N,N'-Bicarbazole-Based Dopant-Free Hole Transporting Materials for Large-Area Perovskite Solar Cells. Adv. Energy Mater. 2018, 8, 1800538.

(S3) Karakawa, M.; Aso, Y. Narrow-Optical-Gap $\pi$-Conjugated Small Molecules Based on Terminal Isoindigo and Thienoisoindigo Acceptor Units for Photovoltaic Application. RSC Adv. 2013, 3, 16259-16263.

(S4) Techajaroonjit, T.; Namuangruk, S.; Prachumrak, N.; Promarak, V.; Sukwattanasinitt, M.; Rashatasakhon, P. Synthesis, Characterization, and Hole-Transporting Properties of Pyrenyl $N$ Substituted Triazatruxenes. RSC Adv. 2016, 6, 56392-56398.

(S5) Bulut, I.; Lévêque, P.; Heinrich, B.; Heiser, T.; Bechara, R.; Zimmermann, N.; Méry, S.; Ziesseld, R.; Leclerc, N. LUMO's Modulation by Electron Withdrawing Unit Modification in Amorphous TAT Dumbbell-Shaped Molecules. J. Mater. Chem. A 2015, 3, 6620-6628.

(S6) Bulut, I.; Chávez, P.; Mirloup, A.; Huaulmé, Q.; Hébraud, A.; Heinrich, B.; Fall, S.; Méry, S.; Ziessel, R.; Heiser, T.; Lévêque, P.; Leclerc, N. Thiazole-Based Scaffolding for High Performance Solar Cells. J. Mater. Chem. C 2016, 4, 4296-4303.

(S7) Becke, A. D. A New Mixing of Hartree-Fock and Local Density-Functional Theories. J. Chem. Phys. 1993, 98, 1372-1377.

(S8) Lynch, B. J.; Fast, P. L.; Harris, M.; Truhlar, D. G. Adiabatic Connection for Kinetics. J. Phys. Chem. A 2000, 104, 4811-4815.

(S9) Cossi, M.; Rega, N.; Scalmani, G.; Barone, V. Energies, Structures, and Electronic Properties of Molecules in Solution with the C-PCM Solvation Model. J. Comput. Chem. 2003, 24, 669-681. 
(S10) Wang, P.; Zakeeruddin, S. M.; Comte, P.; Charvet, R.; Humphry-Baker, R.; Grätzel, M. Enhance the Performance of Dye-Sensitized Solar Cells by Co-grafting Amphiphilic Sensitizer and Hexadecylmalonic Acid on $\mathrm{TiO}_{2}$ Nanocrystals. J. Phys. Chem. B 2003, 107, 14336-14341.

(S11) Hexemer, A.; Bras, W.; Glossinger, J.; Schaible, E.; Gann, E.; Kirian, R.; MacDowell, A.; Church, M.; Rude, B.; Padmore, H. A SAXS/WAXS/GISAXS Beamline with Multilayer Monochromator. J. Phys. Conf. Ser. 2010, 247, 012007.

(S12) Rivnay, J.; Mannsfeld, S. C. B.; Miller, C. E.; Salleo, A.; Toney, M. F. Quantitative Determination of Organic Semiconductor Microstructure from the Molecular to Device Scale. Chem. Rev. 2012, 112, 5488-5519. 